\title{
GERADOR GRÁFICO DE SUBESTRUTURAS CONSTITUÍDAS DE ELEMENTOS LINEARES
}

Eng. José Fernando Moretti

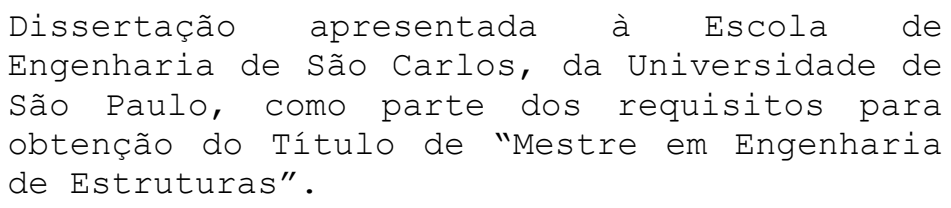

ORIENTADORA: Profa. Dra. Helena M. C. Carmo Antunes

\section{SÃO CARLOS}




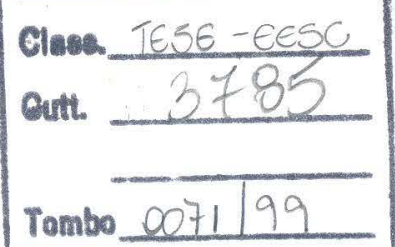

Ficha catalográfica preparada pela Seção de Tratamento da Informação do Serviço de Biblioteca - EESC/USP

Moretti, José Fernando

M845g Gerador gráfico de subestruturas constituídas de elementos lineares / José Fernando Moretti. -- São Carlos, 1998.

Dissertação (Mestrado) -- Escola de Engenharia de São Carlos-Universidade de São Paulo, 1998.

Área: Engenharia de Estruturas.

Orientador: Profa. Dra. Helena M. C. Carmo Antunes.

1. Gerador gráfico. 2. Estruturas lineares.

3. Subestruturas. I. Título. 
Ao meu pai Mário João, incansável incentivador, dedico este trabalho 


\section{AGRADECIMENTOS}

Á $\operatorname{Prof}_{a}$. Dra Helena M. C. Carmo Antunes por sua orientação sempre segura e objetiva, tornou possível a realização deste trabalho.

A todos os professores e amigos do Departamento de Engenharia de Estruturas EESC-USP, que proporcionaram um ambiente edificante, em todos os sentidos.

Ao Departamento de Engenharia Civil da FEIS-UNESP, sempre solícito nos momentos necessários.

À instituição UNESP - Universidade Estadual Paulista, por possibilitar a execução deste trabalho. 


\section{SUMÁRIO}

LISTA DE FIGURAS................... LISTA DE TABELAS.....................

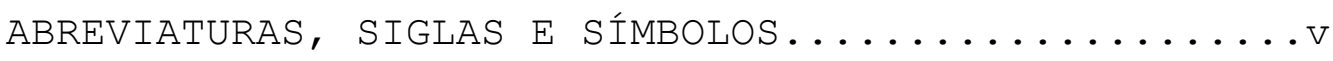
RESUMO $\ldots \ldots \ldots \ldots \ldots \ldots \ldots \ldots \ldots \ldots \ldots \ldots \ldots \ldots \ldots \ldots \ldots$ ABSTRACT..............................

$1 \quad$ - Introdução.......................

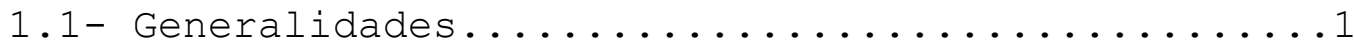

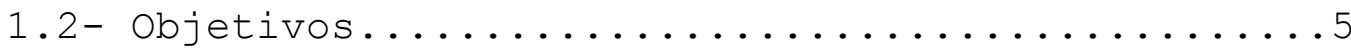

2. - Subestruturas...................

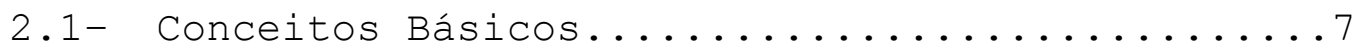

2.2- Método dos Deslocamentos.................8

2.3- Matriz de Rigidez Condensada..............10

2.4- Método "Full Release"..................14

2.5- Método "Cholesky Decomposition"...........20

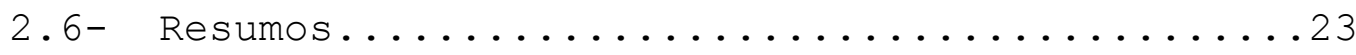

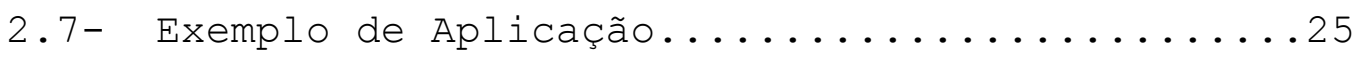

3. - Implementação do Gerador Gráfico............28

3.1- Sistema de Referência Global..............28

3.2- Características das subestruturas..........29

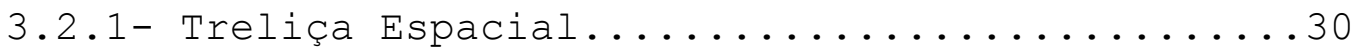




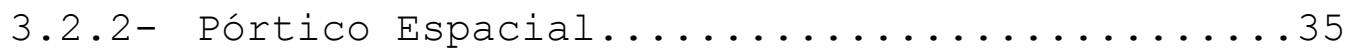

3.3- Formação das Subestruturas..............42

3.4- Renumeração das Coordenadas.............44

3.5- Matrizes Condensadas................47

3.6- Solução dos Deslocamentos Externos.........47

3.7- Deslocamentos Internos.................50

3.8- Técnicas Computacionais................52

3.9- Subrotinas Principais e Fluxograma..........54

3.10- Interfaces Para Aquisição de Dados.........57

3.10.1- Discretização de uma subestrutura.......58

3.10.2- Características das barras...........60

3.10.3- Cargas aplicadas nos nós............61

3.10.4- Cargas aplicadas nas barras..........63

3.10.5- Restrições de apoios.............65

3.10 .6 - Outras funções................66

$3.10 .7-$ Arquivos de dados................68

$3.11-$ Editor em modo texto................69

4. - Exemplos...................... 72

4.2- Exemplo-1 (Pórtico e treliça)............73

4.3- Exemplo-2 (Prédio com 8 pavimentos)........82

4.4- Exemplo-3 (Galpão Industrial)............90

5. - Conclusões Finais...................100

Referências Bibliográficas...............103 


\section{LISTA DE FIGURAS}

FIGURA 1.1 - Galpão Industrial...............

FIGURA 1.2 - Treliça apoiada em pilar............4

FIGURA 2.1 - Divisão em subestruturas............8

FIGURA 2.2 - Estrutura particionada.............10

FIGURA 2.3 - Divisão em subestruturas............14

FIGURA 2.4 - Reações de apoio artificiais(tracejado).16

FIGURA 2.5 - Somatória das ações nos nós de união....19

FIGURA 2.6 - Pilar e treliça.................25

FIGURA 3.1 - Sistema de referência global..........29

FIGURA 3.2 - Elemento de treliça espacial que se desenvolve pelo eixo $\mathrm{X}_{\mathrm{L}} \ldots \ldots \ldots \ldots \ldots$

FIGURA 3.3 - Ações no elemento i em relação aos eixos do sistema estrutural (xyz) .....31

FIGURA 3.4 - Relação entre os eixos locais e os do sistema estrutural.............31

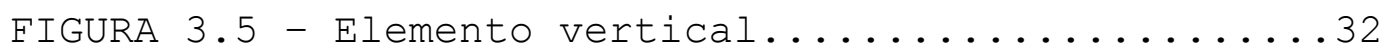

FIGURA 3.6 - Forças aplicadas em nó de treliça......35

FIGURA 3.7 - Elemento de pórtico espacial que se desenvolve pelo seu eixo local $\mathrm{X}_{\mathrm{L}} \ldots \ldots 35$

FIGURA 3.8 - Relação entre eixos locais e globais, incluindo angulo $\alpha$ de rotação da seção transversal em torno de $\mathrm{X}_{\mathrm{L}} \ldots 37$

FIGURA 3.9 - Relação entre eixos locais e eixos globais para elemento de pórtico.....37

FIGURA 3.10 - Carregamentos aplicados diretamente

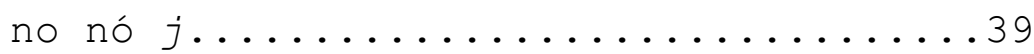

FIGURA 3.11 - Carregamento no plano $\mathrm{X}_{\mathrm{L}}-\mathrm{Y}_{\mathrm{L}} \ldots \ldots \ldots 40$

FIGURA 3.12 - Carregamento no plano $\mathrm{X}_{\mathrm{L}}-\mathrm{Z}_{\mathrm{L}} \ldots \ldots \ldots . .41$ FIGURA 3.13 - Sistema estrutural com pórtico 


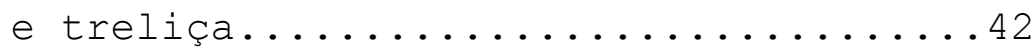

FIGURA 3.14 - Sistema estrutural dividido em subestrutura com numeração dos nós....43

FIGURA 3.15 - Nós das subestruturas com coordenadas iguais...............44

FIGURA 3.16 - Renumeração das coordenadas, da original(a) para a nova(b) ........46 FIGURA 3.17 - Nós de união numerados para formação da $2^{a}$ etapa do processo...........48

FIGURA 3.18 - Coordenadas das subestruturas a serem acopladas ao sistema de equações.....49

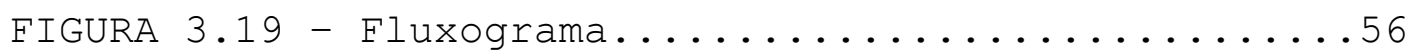

FIGURA 3.20 - Ícones (comandos), em destaque, acrescidos ao programa Autocad, e subestrutura selecionada

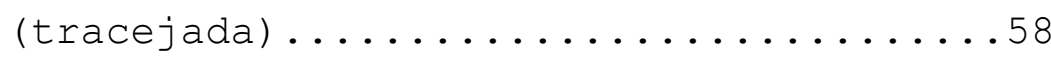

FIGURA 3.21 - Nós selecionados em amarelo para receberem carregamentos...........62

FIGURA 3.22 - Barras selecionadas para receberem carregamentos.................64

FIGURA 3.23 - Nós de apoio setados em azul.........65 FIGURA 3.24 - Novas subestruturas que foram criadas pelo processo de "scopy".........67

FIGURA 3.25 - Editor de texto e Ajuda durante a edição de "Gnos"................71

FIGURA 4.1 - União pórtico e treliça.............73 FIGURA 4.2 - Características do pilar............73 FIGURA 4.3 - Características da treliça...........73 FIGURA 4.4 - Barras selecionadas para definição da subestrutura treliça..........74

FIGURA 4.5 - Nós selecionados para receberem carregamentos.................75 
FIGURA 4.6 - Estrutura completa idealizada com a união de subestruturas já discretizadas ...76

FIGURA 4.7 - Deslocamentos característicos de cada subestrutura nos nós de união......76

FIGURA 4.8 - Prédio com 8 pavimentos e sua orientação global...............82

FIGURA 4.9 - Pavimento tipo, constituindo-se numa subestrutura.............83

FIGURA 4.10 - Características do pilar...........83

FIGURA 4.11 - Principais subestruturas já definidas...84

FIGURA 4.12 - Pilares definidos por "scopy"........84

FIGURA 4.13 - Estrutura completa formada pela união de subestruturas já definidas......85

FIGURA 4.14 - Compatibilizar 6 deslocamentos em cada nó de união, entre pilares e vigas....86

FIGURA 4.15 - Deslocamentos horizontais..........88

FIGURA 4.16 - Estrutura formada por pórticos,

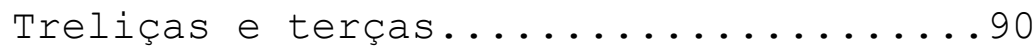

FIGURA 4.17 - Numeração dos nós da treliça.........90

FIGURA 4.18 - Numeração dos nós do pórtico.........90

FIGURA 4.19 - Numeração dos nós da Terça...........91

FIGURA 4.20 - Discretização da treliça............91

FIGURA 4.21 - Treliças em cor azul e pórticos

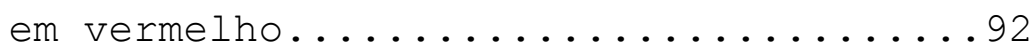

FIGURA 4.22 - Estrutura completa, formada pela união das subestruturas já definidas.......93 


\section{LISTA DE TABELAS}

TABELA 3.1 - Valores dos vetores auxiliares para a subestrutura $\mathrm{P} 1 \ldots \ldots \ldots 46$

TABELA 4.1 - Resultados pilar P1..............77

TABELA 4.2 - Resultados pilar P2.............78

TABELA 4.3 - Resultados pilar P3..............78

TABELA 4.4 - Resultados Treliçal.............79

TABELA 4.5 - Resultados Treliça2............80

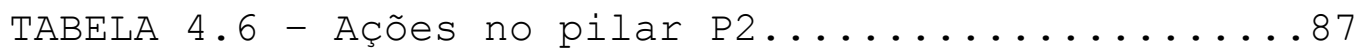

TABELA 4.7 - Deslocamentos no pilar P2.........87

TABELA 4.8 - Ações na viga V4 do pavimento 4.....88

TABELA 4.9 - Deslocamentos na viga v4 pavimento 4...89 


\section{ABREVIATURAS, SIGLAS E SÍMBOLOS}

As principais abreviaturas e símbolos utilizadas neste trabalho são:

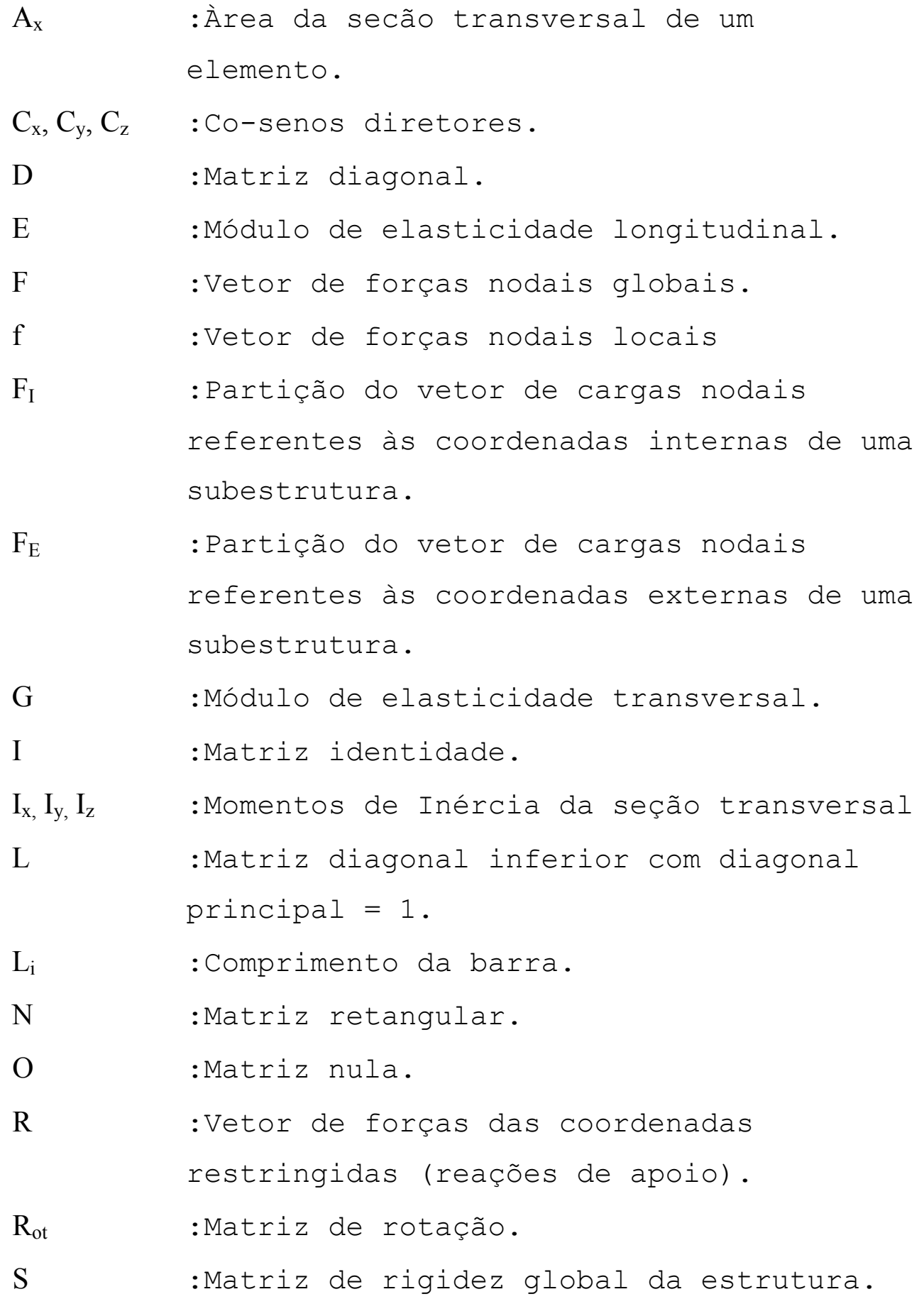


:Partição da matriz de rigidez de uma subestrutura, relacionando ações em coordenadas externas devido a deslocamentos unitários nas mesmas coordenadas externas.

$\mathrm{S}_{\mathrm{IE}} \quad$ : Partição da matriz de rigidez de uma subestrutura, relacionando ações em coordenadas internas devido a deslocamentos unitários em coordenadas externas.

$\mathrm{S}_{\text {II }}$ :Partição da matriz de rigidez de uma subestrutura, relacionando ações em coordenadas internas devido a deslocamentos unitários das mesmas coordenadas internas.

$\mathrm{S}_{\mathrm{M}}$ :Matriz de rigidez de membro.

$\mathrm{S}_{\mathrm{MD}} \quad$ :Matriz de rigidez de membro para os eixos da estrutura (rotacionada).

$\mathrm{S}_{\mathrm{S}} \quad$ :Matriz de rigidez da subestrutura.

U :Vetor deslocamentos globais.

$\mathrm{U}_{\mathrm{I}} \quad$ :Partição do vetor deslocamentos globais, referentes às coordenadas internas.

$\mathrm{U}_{\mathrm{E}} \quad$ :Partição do vetor deslocamentos globais, referentes às coordenadas externas. [] : Indica matriz ou vetor(matriz unitária).

Demais símbolos utilizados são identificados no transcorrer do trabalho, quando da sua primeira utilização ou quando se fizer necessário. 


\section{RESUMO}

Moretti, J. F. (1988). Gerador gráfico de subestruturas constituídas de elementos lineares. São Carlos, 80p. Dissertação (mestrado)

- Escola de Engenharia de São Carlos, Universidade de São Paulo.

Este trabalho consiste na implementação de um gerador gráfico que propiciará a discretização de estruturas lineares formadas pela união de subestruturas.

Estruturas com muitos graus de liberdade serão calculadas através da sua divisão em subestruturas, permitindo uma significativa diminuicão nas dimensões das matrizes envolvidas.

A aplicação da técnica das subestruturas irá permitir, também, a análise de diferentes tipos estruturais interligados, como pórticos e treliças, compatibilizando os deslocamentos comuns nos nós de união.

- gerador gráfico irá tratar as subestruturas como sendo entidades próprias, isoladas, conferindo a elas características que possibilitarão transformações gráficas durante a sua edição.

Pretende-se que o gerador gráfico condicione a uma melhor organização na discretização estrutural, como também venha a facilitar todo o processo de lançamento da estrutura.

Do mesmo modo, cria condições para submeter adequadamente os resultados de cálculo a outros programas, como os de dimensionamento e detalhamento.

Palavras-chave: Gerador gráfico; Estruturas Lineares; Subestruturas. 


\section{ABSTRACT}

Moretti, J. F. (1988). Graphic generator of substructures constituted by lineal elements. . São Carlos, 80p. Dissertação (mestrado) - Escola de Engenharia de São Carlos, Universidade de São Paulo.

This work consists of the implementation of a graphic generator that will propitiate the discretization of lineal structures formed by the substructures union.

Structures with many degrees of freedom will be calculated through its division in substructures, allowing a significant decrease in the dimensions of the involved matrix.

The application of the technique of the substructures will allow, also, the analysis of different interlinked structural types, as frames and trusses, based on the compatibility of the common displacements in the union knots.

The graphic generator will treat the substructures as being own, isolated entities, checking them characteristics that will facilitate graphic transformations during its edition.

It is intended that the graphic generator conditions to a better organization in the structural discretization, as well as come to facilitate the whole process of release of the structure.

In the same way, it creates conditions to submit the calculation results appropriately the other programs, as the one of design and details.

Keywords: Graphic generator; Lineal structures; Substructures. 


\section{CAPÍTULO 1 - INTRODUÇÃO}

\section{1 - Generalidades}

Com a ampla utilização de microcomputadores, nos dias de hoje, torna-se fácil proceder à análise estrutural por meio destes, o que se constitui na prática corriqueira nos escritórios de cálculo estrutural.

Essa realidade se firmou mediante constantes avanços tecnológicos, permitindo drásticas diminuições no valor comercial dos equipamentos e de seus periféricos.

Paralelamente, ocorreram também progressos fundamentais no desenvolvimento de sistemas operacionais, programas, ou seja, em toda lógica envolvida, em geral, nos softwares. Esses fatos contribuíram para que a análise estrutural acompanhasse de perto estes avanços.

A análise estrutural, em sua forma matricial teve seu desenvolvimento no início do século. Era impraticável, devido ao grande número de equações na sua resolução normal, até para estruturas mais simples. Mesmo assim vislumbrou-se a capacidade que esta forma propiciava de fornecer meios adequados de cálculo. 
Com o aparecimento dos computadores, o aprimoramento do método das forças e do método dos deslocamentos, na forma matricial, tem seu reinício, resultando com isto, todas as vantagens inerentes ao processo, como: rapidez nos cálculos, precisão numérica e melhor descrição do comportamento estrutural.

A implementação desses métodos em computadores também passa por constantes evoluções, visando a otimização no desempenho e na utilização de memória do computador.

Cabe lembrar que antes do advento da informática, era quase impraticável o cálculo estrutural tridimensional, mesmo para pequenas estruturas convencionais. Perdia-se precisão nos fenômenos físicos envolvidos.

Grandes estruturas requerem a solução de grandes sistemas de equações, exigindo quantidades significativas de memória no computador. Mais uma vez, a análise tridimensional generalizada das estruturas precisa ser otimizada.

Desta forma, a evolução nesse campo também se direciona à formulação de técnicas que otimizem a utilização da memória disponível no computador.

A Técnica da Subestruturação baseia-se na divisão de uma estrutura em estruturas menores (subestruturas), que podem ser analisadas individualmente, como se fossem isoladas.

Foi introduzido pela primeira vez na análise de circuitos elétricos e estruturas elásticas. Posteriormente a engenharia estrutural o definiria como método das subestruturas.

Esta técnica requer a formulação de todo um procedimento a ser implementado, originando etapas que 
deverão ser convenientemente interligadas, mantendo as características físicas e geométricas da estrutura original. Matematicamente, é vista como a solução de um sistema de equações na forma matricial particionada.

Sua aplicação para a engenharia de estruturas obteve um grande impulso com PRZEMIENIECKI(1968) e Weaver(1967), na formulação do processo, hoje denominado de "FULL RELEASE" .

Posteriormente, RUBINSTEIN(1970) aprimorou esta técnica com melhorias em sua formulação matemática, denominada de "CHOLESKY DECOMPOSITION". Requer menor quantidade de cálculos numéricos a serem efetuados.

Desta forma, amplia-se mais a importância e a aplicação desta técnica, permitindo o cálculo de estruturas com significativa quantidade de graus de liberdade.

Outra vantagem, advinda com esse método, diz respeito a grandes sistemas estruturais complexos, que, além de apresentarem enorme quantidade de graus de liberdade, caracterizam-se por conter diversos tipos de elementos estruturais interligados (subestruturas), tais como pórticos planos e espaciais, treliças planas e espaciais e vigas. Exemplo disto pode ser a estrutura de um galpão industrial, conforme FIGURA 1.1 . 


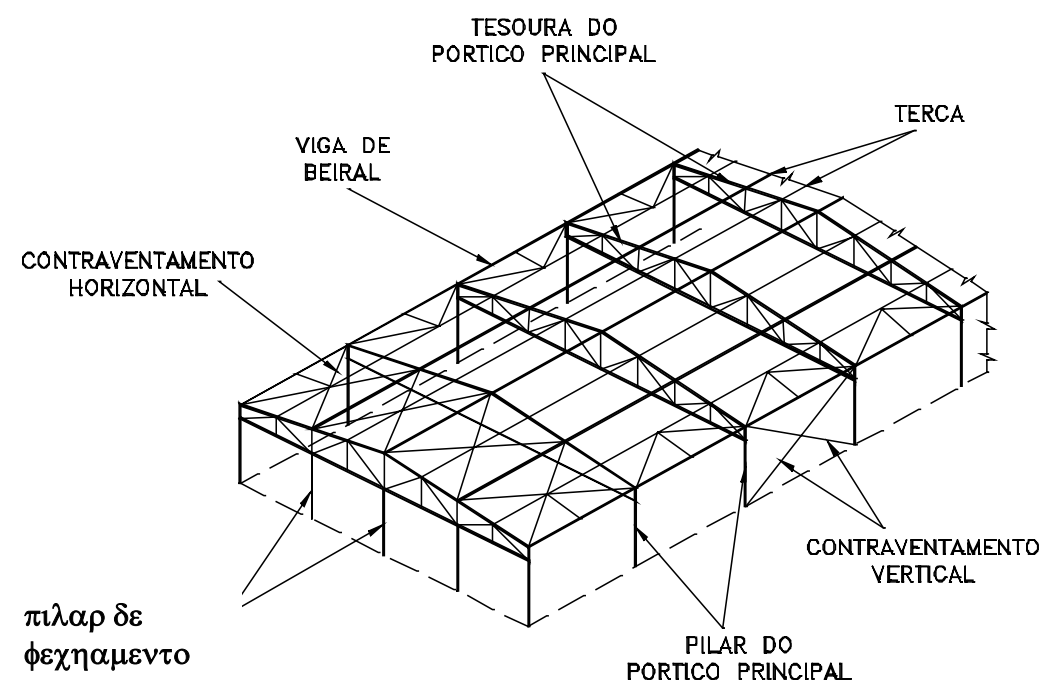

FIGURA 1.1 - Galpão Industrial

A maioria dessas diferentes subestruturas estendemse num plano, como também seus carregamentos, possibilitando com isso uma análise individual de cada uma delas. Durante essa análise leva-se em consideração as ações entre elas, que surgem nos pontos de união. Essa é uma prática que ocorre normalmente, mesmo com a análise estrutural informatizada.

Exemplificando esta prática, temos um pilar de um pórtico apoiando uma treliça, ambos constituídos por elementos estruturais diferentes, mas com algumas solicitações em comum no ponto de união.
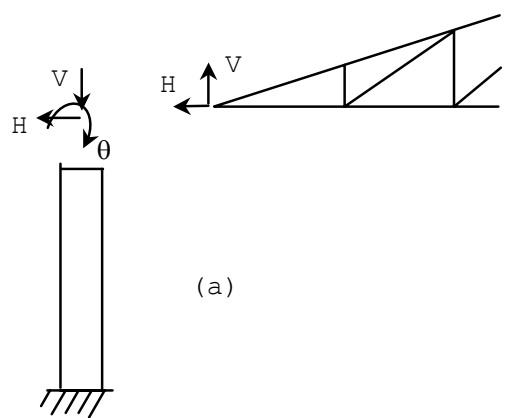

(a)

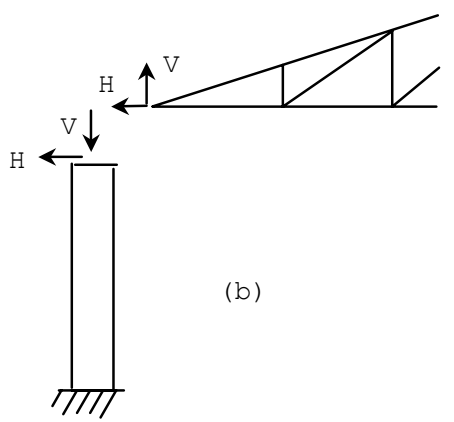

FIGURA 1.2 - Treliça apoiada em pilar 
A figura 1.2 (a) mostra os deslocamentos típicos que ocorrem em um pórtico no plano e numa treliça plana, isoladamente. A figura 1.2 (b) mostra os deslocamentos em comuns entre essas duas estruturas.

No procedimento normal, descrito acima, podemos primeiramente calcular a treliça, obtendo as reações correspondentes a $\mathrm{H}$ e $\mathrm{V}$, necessárias ao seu equilíbrio, e posteriormente introduzi-las como cargas aplicadas no pilar, durante sua análise.

Procedimento semelhante acontece também ao se calcular isoladamente uma terça apoiada nos nós de uma treliça.

Estas simplificações, aplicadas com bom senso, produzem resultados utilizáveis. Porém, maior precisão viria da compatibilização dos deslocamentos comuns nos nós de união.

Maior dificuldade se encontra na determinação mais exata dos esforços envolvendo elementos de contraventamento, que por estarem dispostos na estrutura de modo a redistribuir esforços, influenciando várias outras subestruturas ao mesmo tempo.

A análise tridimensional, de uma estrutura deste tipo, resulta numa melhor descrição dos fenômenos envolvidos, por compatibilizar os deslocamentos entre as várias subestruturas.

\section{2 - Objetivos}

Baseado no exposto acima, o objetivo deste trabalho é implementar a Técnica da Subestruturação em um programa para análise estrutural. 
Será idealizado de modo a conter sub-rotinas direcionadas para tratamento de estruturas aporticadas no espaço e para treliças espaciais. Realizará a compatibilização dos deslocamentos comuns entre elas.

Dessa forma, contempla-se todos os tipos de elementos de barra que possam ocorrer numa estrutura.

Cada subestrutura, uma treliça por exemplo, será discretizada isoladamente. O programa se encarregará de unir todas as outras para formar a estrutura principal, através da detecção dos nós em comum, ou seja, que tenham continuidade de deslocamentos segundo as mesmas coordenadas.

Cada subestrutura será adequadamente tratada, segundo o seu tipo estrutural, pórtico ou treliça. Em seus nós de união serão compatibilizados os deslocamentos em comum.

Pretende-se não somente a otimização do uso da memória do computador, mas também organizar melhor a idealização do sistema formado por todas as subestruturas.

Será utilizado o método dos deslocamentos na formulação de todas as etapas deste trabalho.

o programa, que realizará a análise matricial das estruturas, será elaborado utilizando compilador para a linguagem de programação PASCAL.

A geração gráfica das estruturas será realizada por um editor gráfico (AutoCad). Posteriormente, sub-rotinas desenvolvidas em AutoLisp irão acrescentar informações no desenho gerado, caracterizando-o como uma estrutura, e fornecerão tais informações ao programa de análise estrutural. 


\section{CAPÍTULO 2 - SUBESTRUTURAS}

\section{1 - Conceitos básicos}

A técnica da subestruturação é uma das mais convenientes para o tratamento de estruturas complexas, com muitos graus de liberdade.

Ela consiste na divisão da estrutura completa, de modo a formar segmentos, subestruturas, que podem ser analisadas individualmente como se fossem únicas e independentes.

A FIGURA 2.1 mostra uma estrutura completa, que foi dividida em subestruturas $\mathrm{S} 1, \mathrm{~S} 2$ e $\mathrm{S} 3$. Nota-se que os nós de união entre elas são inicialmente engastados, como parte do processo. 

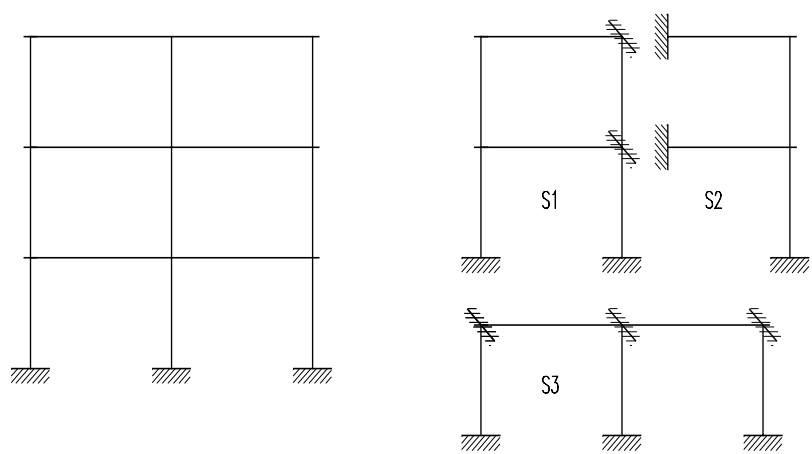

FIGURA 2.1 - Divisão em subestruturas

As subestruturas podem ser definidas como um elemento ou grupo de elementos que formam uma porção da estrutura completa. Cada uma delas, S1 por exemplo, será analisada individualmente, como se fosse única. Para isto, há a formulação de todo um procedimento a ser implementado, originando etapas que deverão ser convenientemente interligadas durante o processo.

$\mathrm{Na}$ solução de cada subestrutura, o número de equações e a quantidade de memória utilizada decaem consideravelmente, comparada com a solução direta, da estrutura completa.

\section{2 - Método dos deslocamentos}

$\mathrm{Na}$ implementação da análise de qualquer sistema físico é necessário a formulação de um modelo matemático descritivo do sistema.

Tal modelo a ser idealizado deve abranger a maioria das estruturas que ocorrem freqüentemente, ou seja, a das estruturas com grande indeterminação estática. 
Das técnicas para a análise elástica de um sistema estrutural indeterminado, o método dos deslocamentos torna-se o preferido, por permitir uma implementação computacional mais genérica.

Este modelo tem como característica principal o seu desenvolvimento com base numa relação linear, existente entre cargas aplicadas e os deslocamentos resultantes na estrutura, tornando válida, então, a aplicação do princípio da superposição.

Conseqüentemente, o material que compõe a estrutura deve seguir a Lei de Hooke e não ultrapassar o limite de elasticidade.

As equações de equilíbrio podem ser desenvolvidas usando o modelo geométrico da estrutura não deformada, baseado em que as deformações que ocorrem na estrutura não são significativas.

Este trabalho segue o modelo descrito acima, utilizando o método dos deslocamentos.

Para uma análise estrutural, submetidas apenas a carregamentos, este método pode ser resumido em:

- Equação geral para deslocamentos nodais.

- Determinação das ações de extremidade dos membros.

- Determinação das reações de apoio.

O desenvolvimento matricial básico deste método não será exposto aqui, devido a farta literatura existente e a predominância na utilização deste método na grande maioria de trabalhos publicados. 


\section{3 - Matriz de rigidez condensada}

A formulação da técnica da subestruturação tem como premissa básica o conceito de condensação da matriz de rigidez e do vetor de forças nodais.

Considere a estrutura fictícia (FIGURA 2.2), dividida em 2 regiões, A e B.

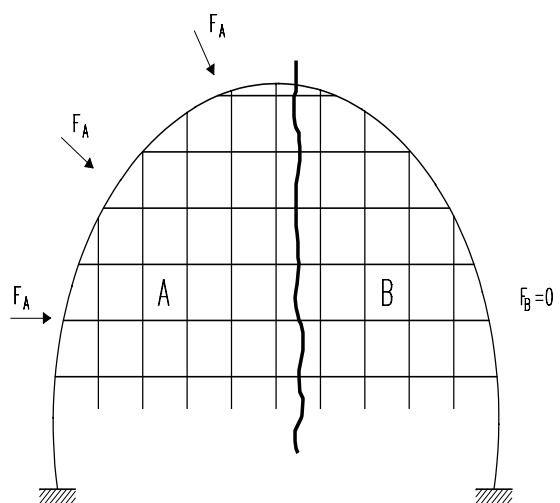

FIGURA 2.2 - Estrutura particionada

Por conveniência, cargas são aplicadas na região A.

Se, somente os deslocamentos nesta região são os de interesse, é preferível gerar apenas uma matriz de rigidez referente a esta região.

A equação de equilíbrio envolvendo toda a estrutura completa, em sua forma particionada é:

$\left[\begin{array}{ll}\mathrm{S}_{\mathrm{AA}} & \mathrm{S}_{\mathrm{AB}} \\ \mathrm{S}_{\mathrm{BA}} & \mathrm{S}_{\mathrm{BB}}\end{array}\right]\left[\begin{array}{c}\mathrm{U}_{\mathrm{A}} \\ \mathrm{U}_{\mathrm{B}}\end{array}\right]=\left[\begin{array}{l}\mathrm{F}_{\mathrm{A}} \\ \mathrm{F}_{\mathrm{B}}\end{array}\right]$

$\mathrm{F}$ = Carregamentos nodais equivalentes.

$\mathrm{S}=$ Matriz de Rigidez

$\mathrm{U}=$ Deslocamentos.

$\mathrm{A}$ e $\mathrm{B}=$ Índices para região da estrutura. 
$\operatorname{Com} \mathrm{F}_{\mathrm{A}} \neq 0$ e $\mathrm{F}_{\mathrm{B}}=0$

A região B na estrutura não contém forças aplicadas.

$\operatorname{De}(2.1):$

$$
\begin{aligned}
& \mathrm{S}_{\mathrm{BA}} \mathrm{U}_{\mathrm{A}}+\mathrm{S}_{\mathrm{BB}} \mathrm{U}_{\mathrm{B}}=0 \\
& \mathrm{~S}_{\mathrm{AA}} \mathrm{U}_{\mathrm{A}}+\mathrm{S}_{\mathrm{AB}} \mathrm{U}_{\mathrm{B}}=\mathrm{F}_{\mathrm{A}}
\end{aligned}
$$

$\operatorname{De}(2.2):$

$$
\mathrm{U}_{\mathrm{B}}=-\mathrm{S}_{\mathrm{BB}}^{-1} \mathrm{~S}_{\mathrm{BA}} \mathrm{U}_{\mathrm{A}}
$$

O termo $\mathrm{S}_{\mathrm{BA}} \mathrm{U}_{\mathrm{A}}$ em (2.4) resulta forças na região $\mathrm{B}$ devido aos deslocamentos na região $A$, e que estas forças causarão deslocamentos $\mathrm{U}_{\mathrm{B}}$, região $\mathrm{B}$, conforme a equação (2.4) completa.

Substituindo (2.4) em (2.3):

$$
\begin{aligned}
& \mathrm{F}_{\mathrm{A}}=\mathrm{S}_{\mathrm{AA}} \mathrm{U}_{\mathrm{A}}+\mathrm{S}_{\mathrm{AB}}\left(-\mathrm{S}_{\mathrm{BB}}^{-1} \mathrm{~S}_{\mathrm{BA}} \mathrm{U}_{\mathrm{A}}\right) \\
& \mathrm{F}_{\mathrm{A}}=\left(\mathrm{S}_{\mathrm{AA}}-\mathrm{S}_{\mathrm{AB}} \mathrm{S}_{\mathrm{BB}}^{-1} \mathrm{~S}_{\mathrm{BA}}\right) \mathrm{U}_{\mathrm{A}} \\
& \mathrm{F}_{\mathrm{A}}=\mathrm{S}_{\mathrm{C}} \mathrm{U}_{\mathrm{A}}
\end{aligned}
$$

Onde

$$
\mathrm{S}_{\mathrm{C}}=\left(\mathrm{S}_{\mathrm{AA}}-\mathrm{S}_{\mathrm{AB}} \mathrm{S}_{\mathrm{BB}}^{-1} \mathrm{~S}_{\mathrm{BA}}\right) \text { é a matriz de rigidez }
$$

condensada.

Finalmente, de (2.5):

$$
\mathrm{U}_{\mathrm{A}}=\mathrm{S}_{\mathrm{C}}^{-1} \mathrm{~F}_{\mathrm{A}}
$$


Resolvendo este sistema, podemos então completar a análise da estrutura para a região $A$, que é a de interesse.

A matriz $\mathrm{S}_{\mathrm{C}}$ engloba toda a atuação que a região $\mathrm{B}$ da estrutura exerce sobre as coordenadas em A. Sua dimensão é consideravelmente menor que a da matriz de rigidez da estrutura em sua forma completa.

o termo condensação refere-se, então, a uma contração no tamanho do sistema de equações, eliminando certos graus de liberdade durante o processo (região B), mas computando suas atuações na matriz $\mathrm{S}_{\mathrm{C}}$. Neste processo, nada é aproximado ou perdido.

Em uma situação geral, com aplicação de cargas na região B da FIGURA 2.2, temos a equação de equilíbrio particionada:

$$
\left[\begin{array}{ll}
\mathrm{S}_{\mathrm{AA}} & \mathrm{S}_{\mathrm{AB}} \\
\mathrm{S}_{\mathrm{BA}} & \mathrm{S}_{\mathrm{BB}}
\end{array}\right]\left[\begin{array}{l}
\mathrm{U}_{\mathrm{A}} \\
\mathrm{U}_{\mathrm{B}}
\end{array}\right]=\left[\begin{array}{l}
\mathrm{F}_{\mathrm{A}} \\
\mathrm{F}_{\mathrm{B}}
\end{array}\right]
$$

$\operatorname{Com} \quad \mathrm{F}_{\mathrm{A}} \neq 0$ e $\mathrm{F}_{\mathrm{B}} \neq 0$

Desmembrando:

$$
\begin{aligned}
& \mathrm{S}_{\mathrm{AA}} \mathrm{U}_{\mathrm{A}}+\mathrm{S}_{\mathrm{AB}} \mathrm{U}_{\mathrm{B}}=\mathrm{F}_{\mathrm{A}} \\
& \mathrm{S}_{\mathrm{BA}} \mathrm{U}_{\mathrm{A}}+\mathrm{S}_{\mathrm{BB}} \mathrm{U}_{\mathrm{B}}=\mathrm{F}_{\mathrm{B}}
\end{aligned}
$$

De (2.9):

$$
\mathrm{U}_{\mathrm{B}}=\mathrm{S}_{\mathrm{BB}}^{-1} \mathrm{~F}_{\mathrm{B}}-\mathrm{S}_{\mathrm{BB}}^{-1} \mathrm{~S}_{\mathrm{BA}} \mathrm{U}_{\mathrm{A}}
$$


Substituindo (2.10) em (2.8):

$$
\begin{aligned}
& \mathrm{F}_{\mathrm{A}}=\mathrm{S}_{\mathrm{AA}} \mathrm{U}_{\mathrm{A}}+\mathrm{S}_{\mathrm{AB}}\left(\mathrm{S}_{\mathrm{BB}}^{-1} \mathrm{~F}_{\mathrm{B}}-\mathrm{S}_{\mathrm{BB}}^{-1} \mathrm{~S}_{\mathrm{BA}} \mathrm{U}_{\mathrm{A}}\right) \\
& \mathrm{F}_{\mathrm{A}}-\mathrm{S}_{\mathrm{AB}} \mathrm{S}_{\mathrm{BB}}^{-1} \mathrm{~F}_{\mathrm{B}}=\left(\mathrm{S}_{\mathrm{AA}}-\mathrm{S}_{\mathrm{AB}} \mathrm{S}_{\mathrm{BB}}^{-1} \mathrm{~S}_{\mathrm{BA}}\right) \mathrm{U}_{\mathrm{A}}
\end{aligned}
$$

Sendo:

$$
\begin{aligned}
& \mathrm{S}_{\mathrm{C}}=\left(\mathrm{S}_{\mathrm{AA}}-\mathrm{S}_{\mathrm{AB}} \mathrm{S}_{\mathrm{BB}}^{-1} \mathrm{~S}_{\mathrm{BA}}\right) \\
& \mathrm{F}_{\mathrm{A}}^{*}=\mathrm{F}_{\mathrm{A}}-\mathrm{S}_{\mathrm{AB}} \mathrm{S}_{\mathrm{BB}}^{-1} \mathrm{~F}_{\mathrm{B}}
\end{aligned}
$$

A solução da estrutura completa se dá em duas etapas.

A primeira, para os deslocamentos na região A:

$$
\mathrm{U}_{\mathrm{A}}=\mathrm{S}_{\mathrm{C}}^{-1} \mathrm{~F}_{\mathrm{A}}^{*}
$$

A Segunda para a região B, utilizando (2.10):

$$
\mathrm{U}_{\mathrm{B}}=\mathrm{S}_{\mathrm{BB}}^{-1} \mathrm{~F}_{\mathrm{B}}-\mathrm{S}_{\mathrm{BB}}^{-1} \mathrm{~S}_{\mathrm{BA}} \mathrm{S}_{\mathrm{C}}^{-1} \mathrm{~F}_{\mathrm{A}}^{*}
$$

Todo este sistema de equações desenvolvido acima, mostra a solução de uma grande estrutura que foi dividida em 2 partes, particionando-se a equação de equilíbrio. Com isto, requer-se maior quantidade de operações aritméticas, comparadas à solução direta não particionada. Porém a dimensão das matrizes é menor.

o conceito de condensação foi utilizado, embora não identificado por este termo. 


\section{4 - Método "Full Release"}

A técnica da condensação, aplicada de forma organizada, propicia a formulação geral de métodos para divisão em subestruturas.

O método apresentado por PRZEMIENIECKI(1968), denominado "FULL RELEASE", considerado o método padrão, será desenvolvido aqui para fins ilustrativos e didáticos, utilizando o método dos deslocamentos na análise estrutural.

Considere o pórtico da FIGURA 2.3, dividido em 2 subestruturas :
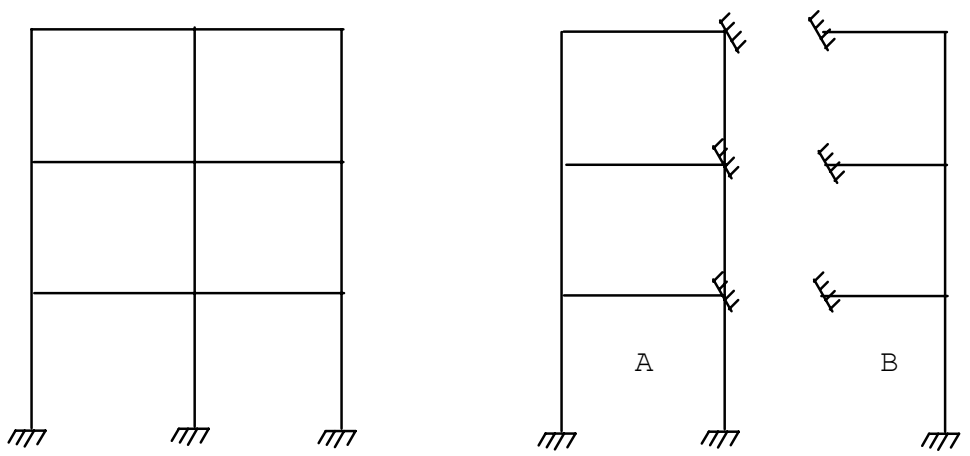

FIGURA 2.3 - Divisão em Subestruturas

Cada subestrutura é considerada isolada do sistema estrutural, como se fossem únicas, com seus nós de contorno restringidos a deslocamentos (engastados). São denominados vínculos artificiais.

Como primeira etapa, ela será analisada com seus carregamentos externos aplicados. A equação de equilíbrio é :

$$
\mathrm{S}^{\mathrm{n}} \mathrm{U}^{\mathrm{n}}=\mathrm{F}^{\mathrm{n}}+\mathrm{R}^{\mathrm{n}}
$$




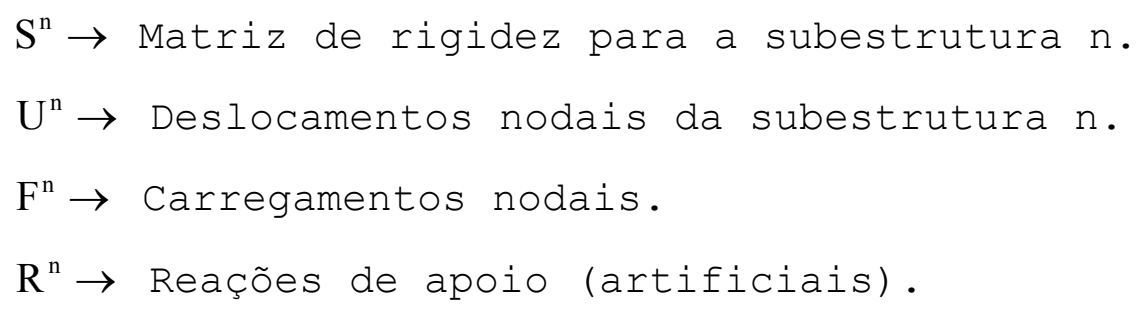

A equação de equilíbrio particionada, com relação aos nós restringido e aos nós livres, fica:

$$
\left[\begin{array}{ll}
\mathrm{S}_{\mathrm{II}}^{\mathrm{n}} & \mathrm{S}_{\mathrm{IE}}^{\mathrm{n}} \\
\mathrm{S}_{\mathrm{EI}}^{\mathrm{n}} & \mathrm{S}_{\mathrm{EE}}^{\mathrm{n}}
\end{array}\right]\left[\begin{array}{c}
\mathrm{U}_{\mathrm{If}}^{\mathrm{n}} \\
0
\end{array}\right]=\left[\begin{array}{c}
\mathrm{F}_{\mathrm{If}}^{\mathrm{n}} \\
\mathrm{F}_{\mathrm{Ef}}^{\mathrm{n}}
\end{array}\right]+\left[\begin{array}{c}
0 \\
\mathrm{R}_{\mathrm{Ef}}^{\mathrm{n}}
\end{array}\right]
$$

O índice I indica as coordenada internas (nós livres). O índice E indica as coordenadas externas, dos nós de união.

O índice f indica esta etapa do processo, onde os nós de união são fixados artificialmente (subestrutura fixada), e cargas externas aplicadas.

$$
\begin{aligned}
& \mathrm{U}_{\mathrm{If}}^{\mathrm{n}} \rightarrow \text { Deslocamentos nos nós livres. } \\
& \mathrm{R}_{\mathrm{EF}}^{\mathrm{n}} \rightarrow \text { Reações nos nós restringidos. }
\end{aligned}
$$

Expandindo a equação (2.16):

$$
\begin{aligned}
& S_{I I}^{n} U_{I f}^{n}=F_{I f}^{n} \\
& S_{E I}^{n} U_{I f}^{n}=F_{E f}^{n}+R_{E f}^{n}
\end{aligned}
$$

Resolvendo a equação (2.17) para os nós livres:

$$
\mathrm{U}_{\mathrm{If}}^{\mathrm{n}}=\mathrm{S}_{\mathrm{II}}^{\mathrm{n}-1} \mathrm{~F}_{\mathrm{If}}^{\mathrm{n}}
$$


Calculando as reações de apoio para a subestrutura fixada, (2.19) em (2.18):

$$
\mathrm{R}_{\mathrm{Ef}}^{\mathrm{n}}=\mathrm{S}_{\mathrm{EI}}^{\mathrm{n}} \mathrm{S}_{\mathrm{II}}^{\mathrm{n}^{-1}} \mathrm{~F}_{\mathrm{If}}^{\mathrm{n}}-\mathrm{F}_{\mathrm{Ef}}^{\mathrm{n}} \quad(2.20)
$$

Esta primeira etapa caracteriza-se pela determinação de $\mathrm{R}_{\mathrm{Ef}}^{\mathrm{n}}$ nos nós de contorno (vínculos artificiais), quando o carregamento é aplicado, FIGURA 2.4.

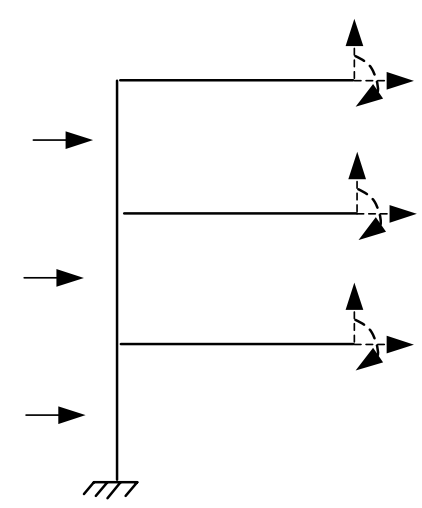

FIGURA 2.4 - Reações de apoio artificiais (tracejado)

Observando que desta análise não resultam ações e deslocamentos verdadeiros nos nós internos das subestruturas.

A segunda etapa do processo consiste em saber a resposta da subestrutura quando sujeita somente aos deslocamentos dos nós de contorno, sem aplicação de qualquer carregamento externo, ou seja:

$$
\mathrm{F}_{\mathrm{I}}^{\mathrm{n}}=\mathrm{F}_{\mathrm{E}}^{\mathrm{n}}=0
$$

A equação de equilíbrio particionada fica: 


$$
\left[\begin{array}{ll}
\mathrm{S}_{\mathrm{II}}^{\mathrm{n}} & \mathrm{S}_{\mathrm{IE}}^{\mathrm{n}} \\
\mathrm{S}_{\mathrm{EI}}^{\mathrm{n}} & \mathrm{S}_{\mathrm{EE}}^{\mathrm{n}}
\end{array}\right]\left[\begin{array}{c}
\mathrm{U}_{\mathrm{Id}}^{\mathrm{n}} \\
\mathrm{U}_{\mathrm{Ed}}^{\mathrm{n}}
\end{array}\right]=\left[\begin{array}{c}
0 \\
\mathrm{R}_{\mathrm{Ed}}^{\mathrm{n}}
\end{array}\right]
$$

O índice d indicando esta etapa do processo, ou seja, ocorrendo somente deslocamentos no contorno.

$$
\begin{aligned}
\mathrm{U}_{\mathrm{Id}}^{\mathrm{n}} \rightarrow & \text { Deslocamentos dos nós livres quando } \\
& \text { ocorre deslocamentos dos nós de união. } \\
\mathrm{U}_{\mathrm{Ed}}^{\mathrm{n}} \rightarrow & \text { Representa os deslocamentos reais dos } \\
& \text { nós de contorno que serão determinados em } \\
& \text { outra etapa. } \\
\mathrm{R}_{\mathrm{Ed}}^{\mathrm{n}} \rightarrow & \text { São as ações (reações) nos nós de } \\
& \text { contorno, para manter o equilíbrio da } \\
& \text { subestrutura quando ocorre } \\
& \text { os deslocamentos } \mathrm{U}_{\mathrm{ED}}^{\mathrm{n}} \cdot
\end{aligned}
$$

Expandindo a equação (2.21):

$$
\begin{aligned}
& \mathrm{S}_{\mathrm{II}}^{\mathrm{n}} \mathrm{U}_{\mathrm{Id}}^{\mathrm{n}}+\mathrm{S}_{\mathrm{IE}}^{\mathrm{n}} \mathrm{U}_{\mathrm{Ed}}^{\mathrm{n}}=0 \\
& \mathrm{~S}_{\mathrm{EI}}^{\mathrm{n}} \mathrm{U}_{\mathrm{Id}}^{\mathrm{n}}+\mathrm{S}_{\mathrm{EE}}^{\mathrm{n}} \mathrm{U}_{\mathrm{Ed}}^{\mathrm{n}}=\mathrm{R}_{\mathrm{Ed}}^{\mathrm{n}}
\end{aligned}
$$

Da equação (2.22):

$$
\mathrm{U}_{\mathrm{Id}}^{\mathrm{n}}=-\mathrm{S}_{\mathrm{II}}^{\mathrm{n}-1} \mathrm{~S}_{\mathrm{IE}}^{\mathrm{n}} \mathrm{U}_{\mathrm{Ed}}^{\mathrm{n}}
$$

Substituindo (2.24) em (2.23):

$$
\begin{aligned}
& -S_{E I}^{n} S_{I I}^{n-1} S_{I E}^{n} U_{E d}^{n}+S_{E E}^{n} U_{E d}^{n}=R_{E d}^{n} \\
& \left(S_{E E}^{n}-S_{E I}^{n} S_{I I}^{n-1} S_{I E}^{n}\right) U_{E d}^{n}=R_{E d}^{n}
\end{aligned}
$$


Sendo que:

$$
\mathrm{S}_{\mathrm{s}}^{\mathrm{n}}=\mathrm{S}_{\mathrm{EE}}^{\mathrm{n}}-\mathrm{S}_{\mathrm{EI}}^{\mathrm{n}} \mathrm{S}_{\mathrm{II}}^{\mathrm{n}-1} \mathrm{~S}_{\mathrm{IE}}^{\mathrm{n}}
$$

$\log 0$ :

$$
\mathrm{R}_{\mathrm{Ed}}^{\mathrm{n}}=\mathrm{S}_{\mathrm{s}}^{\mathrm{n}} \mathrm{U}_{\mathrm{Ed}}^{\mathrm{n}} \quad(2.26)
$$

Esta segunda etapa caracteriza-se pela determinação de $\mathrm{R}_{\mathrm{Ed}}^{\mathrm{n}}$ de cada subestrutura.

$\mathrm{S}_{\mathrm{s}}^{\mathrm{n}}$ indica a ação necessária para um deslocamento unitário nas coordenadas dos nós de união, levando em consideração a interação que há com o resto da subestrutura. É uma matriz condensada. Devido ao seu significado, ela é adequadamente denominada de Matriz de Rigidez da Subestrutura.

Uma vez definida a matriz de ações nos nós de contorno $\mathrm{R}_{\mathrm{Ef}}^{\mathrm{n}}$ e da $\mathrm{S}_{\mathrm{s}}^{\mathrm{n}}$, para todas as subestruturas, temos as condições de calcular os deslocamentos nestes nós de união, com a equação de equilíbrio:

$$
\mathrm{S}_{\mathrm{c}} \mathrm{U}_{\mathrm{c}}=\mathrm{F}_{\mathrm{c}}+\mathrm{R}_{\mathrm{c}}
$$

O índice c indica grandezas completas. Ou seja:

$$
\mathrm{S}_{\mathrm{c}}=\sum_{1}^{\mathrm{n}} \mathrm{S}_{\mathrm{s}}^{\mathrm{n}}
$$

Invertendo-se o sinal de $\mathrm{R}_{\mathrm{Ef}}^{\mathrm{n}}$, temos a matriz de carregamentos nodais equivalentes:

$$
\mathrm{F}_{\mathrm{c}}=-\sum_{1}^{\mathrm{n}} \mathrm{R}_{\mathrm{Ef}}^{\mathrm{n}}
$$


Todas estas grandezas são referentes ao sistema formado pelos nós de união, conforme FIGURA 2.5.

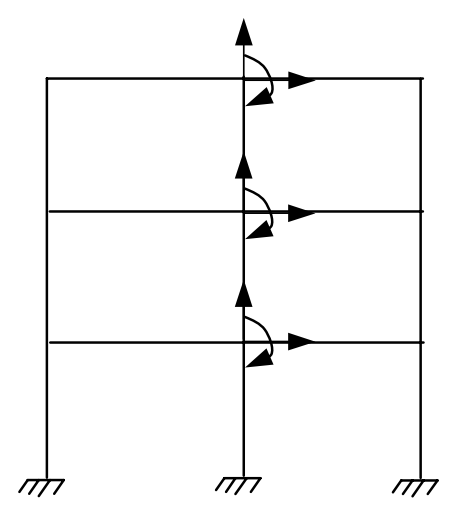

FIGURA 2.5 - Somatória das ações nos nós de união

Resolvendo (2.27):

$$
\mathrm{U}_{\mathrm{c}}=\mathrm{S}_{\mathrm{c}}^{-1} \mathrm{~F}_{\mathrm{c}}
$$

$\mathrm{U}_{\mathrm{c}} \rightarrow$ Deslocamentos que ocorrem nós de união.

Podemos agora calcular os deslocamentos dos nós internos (livres) de cada subestrutura, dado por:

$$
\mathrm{U}_{\mathrm{I}}^{\mathrm{n}}=\mathrm{U}_{\mathrm{If}}^{\mathrm{n}}+\mathrm{U}_{\mathrm{Id}}^{\mathrm{n}}
$$

Ou usando a equação (2.24):

$$
\mathrm{U}_{\mathrm{I}}^{\mathrm{n}}=\mathrm{U}_{\mathrm{If}}^{\mathrm{n}}-\mathrm{S}_{\mathrm{II}}^{\mathrm{n}-1} \mathrm{~S}_{\mathrm{IE}}^{\mathrm{n}} \mathrm{U}_{\mathrm{Ed}}^{\mathrm{n}}
$$


$\mathrm{U}_{\mathrm{ED}}^{\mathrm{n}}$ da subestrutura $\mathrm{n}$ é obtido automaticamente quando da determinação de $\mathrm{U}_{\mathrm{c}}$ •

A análise se completará, para cada subestrutura, determinando-se as ações de extremidade para cada elemento, e as reações de apoios nos vínculos verdadeiros (não artificiais) .

Neste método, verifica-se a presença de uma técnica de relaxação, onde os nós fixados são relaxados simultaneamente.

Esta técnica implica a liberação total dos graus de liberdade internos da subestrutura. A equação (2.19) é dispendiosa em termos computacionais.

As sequiências, aqui apresentadas, caracterizam a formulação do método da subestruturação em paralelo, que é utilizada neste trabalho. Apenas para informação, podese seguir por outra seqüência na formulação do método, denominada subestruturação em série, que não será abordada aqui.

\section{5 - Método "Cholesky Decomposition"}

Posteriormente ao método "FULL RELEASE", a obtenção da matriz de rigidez e vetor de cargas condensadas passou por uma reformulação matemática.

No método "CHOLESKY DECOMPOSITION", apresentado por RUBINSTEIN(1970), envolve a liberação parcial dos graus de liberdade dos nós internos, operado por uma série de transformações que proporcionam um novo conjunto de equações matriciais. 
Tem como grande vantagem a determinação dos deslocamentos dos nós internos depois que os deslocamentos externos são calculados, resultando num processo com menor número de operações numéricas.

Seja a equação matricial de equilíbrio de uma subestrutura:

$$
\left[\begin{array}{ll}
\mathrm{S}_{\mathrm{II}} & \mathrm{S}_{\mathrm{IE}} \\
\mathrm{S}_{\mathrm{EI}} & \mathrm{S}_{\mathrm{EE}}
\end{array}\right]\left[\begin{array}{c}
\mathrm{U}_{\mathrm{I}} \\
\mathrm{U}_{\mathrm{E}}
\end{array}\right]=\left[\begin{array}{c}
\mathrm{F}_{\mathrm{I}} \\
\mathrm{F}_{\mathrm{E}}
\end{array}\right]
$$

Este método pode ser formulado com a decomposição da equação (2.29) no seguinte triplo produto matricial:

$$
\left[\begin{array}{ll}
\mathrm{S}_{\mathrm{II}} & \mathrm{S}_{\mathrm{IE}} \\
\mathrm{S}_{\mathrm{EI}} & \mathrm{S}_{\mathrm{EE}}
\end{array}\right]=\left[\begin{array}{cc}
\mathrm{L} & 0 \\
\mathrm{~N} & \mathrm{I}
\end{array}\right]\left[\begin{array}{cc}
\mathrm{D} & 0 \\
0 & \mathrm{~S}_{\mathrm{EE}}^{*}
\end{array}\right]\left[\begin{array}{cc}
\mathrm{L}^{\mathrm{T}} & \mathrm{N}^{\mathrm{T}} \\
0 & \mathrm{I}
\end{array}\right]
$$

$\mathrm{L}=$ Matriz triangular inferior com diagonais iguais a 1 .

$\mathrm{N}=$ Matriz retangular.

0 = matriz nula.

I = Matriz identidade.

$\mathrm{D}=$ Matriz diagonal.

$\mathrm{S}_{\mathrm{EE}}^{*}=$ Matriz simétrica condensada.

Deste triplo produto matricial, são obtidas as seguintes relações:

$$
\begin{aligned}
& \mathrm{S}_{\mathrm{II}}=\mathrm{LDL}^{\mathrm{T}} \\
& \mathrm{S}_{\mathrm{EI}}=\mathrm{S}_{\mathrm{IE}}^{\mathrm{T}}=\mathrm{NDL}^{\mathrm{T}} \\
& \mathrm{S}_{\mathrm{EE}}=\mathrm{S}_{\mathrm{EE}}^{*}+\mathrm{NDN}^{\mathrm{T}}
\end{aligned}
$$


Relacionando (2.30) com (2.29), podem extrair-se os seguintes sistemas:

$$
\begin{aligned}
& {\left[\begin{array}{cc}
\mathrm{D} & 0 \\
0 & \mathrm{~S}_{\mathrm{EE}}^{*}
\end{array}\right]\left[\begin{array}{c}
\mathrm{U}_{\mathrm{I}}^{*} \\
\mathrm{U}_{\mathrm{E}}^{*}
\end{array}\right]=\left[\begin{array}{c}
\mathrm{F}_{\mathrm{I}}^{*} \\
\mathrm{~F}_{\mathrm{E}}^{*}
\end{array}\right] \quad \begin{array}{l}
\mathrm{DU}_{\mathrm{I}}^{*}=\mathrm{F}_{\mathrm{I}}^{*} \\
\mathrm{~S}_{\mathrm{EE}}^{*} \mathrm{U}_{\mathrm{E}}^{*}=\mathrm{F}_{\mathrm{E}}^{*}
\end{array} \ldots(2.34)} \\
& {\left[\begin{array}{c}
\mathrm{U}_{\mathrm{I}}^{*} \\
\mathrm{U}_{\mathrm{E}}^{*}
\end{array}\right]=\left[\begin{array}{cc}
\mathrm{L}^{\mathrm{T}} & \mathrm{N}^{\mathrm{T}} \\
0 & \mathrm{I}
\end{array}\right]\left[\begin{array}{c}
\mathrm{U}_{\mathrm{I}} \\
\mathrm{U}_{\mathrm{E}}
\end{array}\right] \quad \begin{array}{l}
\mathrm{U}_{\mathrm{I}}^{*}=\mathrm{L}^{\mathrm{T}} \mathrm{U}_{\mathrm{I}}+\mathrm{N}^{\mathrm{T}} \mathrm{U}_{\mathrm{E}} \\
\mathrm{U}_{\mathrm{E}}^{*}=\mathrm{U}_{\mathrm{E}}
\end{array}} \\
& {\left[\begin{array}{c}
\mathrm{F}_{\mathrm{I}} \\
\mathrm{F}_{\mathrm{E}}
\end{array}\right]=\left[\begin{array}{cc}
\mathrm{L} & 0 \\
\mathrm{~N} & \mathrm{I}
\end{array}\right]\left[\begin{array}{l}
\mathrm{F}_{\mathrm{I}}^{*} \\
\mathrm{~F}_{\mathrm{E}}^{*}
\end{array}\right] \quad \begin{array}{l}
\mathrm{F}_{\mathrm{I}}=\mathrm{LF}_{\mathrm{I}}^{*} \\
\mathrm{~F}_{\mathrm{E}}=\mathrm{NF}_{\mathrm{I}}^{*}+\mathrm{F}_{\mathrm{E}}^{*}
\end{array} \ldots(2.36)}
\end{aligned}
$$

Com as novas equações matriciais ...(2.34) a ...(2.36), opera-se o processo de condensação estática por este método, que podem condicionar-se a três etapas:

ETAPA 1: Substituição parcial, combinando as equações ...(2.34) e ...(2.35):

$$
\begin{aligned}
& {\left[\begin{array}{cc}
\mathrm{D} & 0 \\
0 & \mathrm{~S}_{\mathrm{EE}}^{*}
\end{array}\right]\left[\begin{array}{cc}
\mathrm{L}^{\mathrm{T}} & \mathrm{N}^{\mathrm{T}} \\
0 & \mathrm{I}
\end{array}\right]\left[\begin{array}{c}
\mathrm{U}_{\mathrm{I}} \\
\mathrm{U}_{\mathrm{E}}
\end{array}\right]=\left[\begin{array}{c}
\mathrm{F}_{\mathrm{I}}^{*} \\
\mathrm{~F}_{\mathrm{E}}^{*}
\end{array}\right]} \\
& {\left[\begin{array}{cc}
\mathrm{DL}^{\mathrm{T}} & \mathrm{DN}^{\mathrm{T}} \\
0 & \mathrm{~S}_{\mathrm{EE}}^{*}
\end{array}\right]\left[\begin{array}{c}
\mathrm{U}_{\mathrm{I}} \\
\mathrm{U}_{\mathrm{E}}
\end{array}\right]=\left[\begin{array}{c}
\mathrm{F}_{\mathrm{I}}^{*} \\
\mathrm{~F}_{\mathrm{E}}^{*}
\end{array}\right]}
\end{aligned}
$$

ETAPA 2: Solução do sistema de equações condensados, da equação (2.38):

$$
\mathrm{S}_{\mathrm{EE}}^{*} \mathrm{U}_{\mathrm{E}}=\mathrm{F}_{\mathrm{E}}^{*}
$$




$$
\mathrm{IU}_{\mathrm{E}}=\mathrm{U}_{\mathrm{E}}
$$

ETAPA 3: Retrosubstituição parcial, combinando (2.40) e (2.38), ou operando...(2.35), obtem-se:

$$
\begin{aligned}
& {\left[\begin{array}{cc}
\mathrm{DL}^{\mathrm{T}} & \mathrm{DN}^{\mathrm{T}} \\
0 & \mathrm{I}
\end{array}\right]\left[\begin{array}{c}
\mathrm{U}_{\mathrm{I}} \\
\mathrm{U}_{\mathrm{E}}
\end{array}\right]=\left[\begin{array}{c}
\mathrm{F}_{\mathrm{I}}^{*} \\
\mathrm{U}_{\mathrm{E}}
\end{array}\right]} \\
& {\left[\begin{array}{ll}
\mathrm{I} & 0 \\
0 & \mathrm{I}
\end{array}\right]\left[\begin{array}{c}
\mathrm{U}_{\mathrm{I}} \\
\mathrm{U}_{\mathrm{E}}
\end{array}\right]=\left[\begin{array}{c}
\mathrm{U}_{\mathrm{I}} \\
\mathrm{U}_{\mathrm{E}}
\end{array}\right]}
\end{aligned}
$$

\section{6 - Resumos}

As relações matriciais do método "Cholesky Decomposition", quando operadas em forma compacta, como nas ETAPAS 1, 2 e 3, conduzem a aplicação do processo de Gauss em forma parcial.

Adotando:

$$
\begin{aligned}
& \mathrm{DL}^{\mathrm{T}}=\mathrm{S}_{\mathrm{II}}^{*} \\
& \mathrm{DN}^{\mathrm{T}}=\mathrm{S}_{\mathrm{IE}}^{*} \\
& \mathrm{~S}_{\mathrm{EE}}^{*}=\mathrm{S}_{\mathrm{EE}}^{*}
\end{aligned}
$$

ETAPA 1: Substituição parcial até condensar a última equação das coordenadas internas.

$$
\left[\begin{array}{ll}
\mathrm{S}_{\mathrm{II}} & \mathrm{S}_{\mathrm{IE}} \\
\mathrm{S}_{\mathrm{EI}} & \mathrm{S}_{\mathrm{EE}}
\end{array}\right]\left[\begin{array}{c}
\mathrm{U}_{\mathrm{I}} \\
\mathrm{U}_{\mathrm{E}}
\end{array}\right]=\left[\begin{array}{c}
\mathrm{F}_{\mathrm{I}} \\
\mathrm{F}_{\mathrm{E}}
\end{array}\right] \underset{\mathrm{ETAPA} 1}{\Rightarrow}\left[\begin{array}{cc}
\mathrm{S}_{\mathrm{II}}^{*} & \mathrm{~S}_{\mathrm{IE}}^{*} \\
0 & \mathrm{~S}_{\mathrm{EE}}^{*}
\end{array}\right]\left[\begin{array}{c}
\mathrm{U}_{\mathrm{I}} \\
\mathrm{U}_{\mathrm{E}}
\end{array}\right]=\left[\begin{array}{c}
\mathrm{F}_{\mathrm{I}}^{*} \\
\mathrm{~F}_{\mathrm{E}}^{*}
\end{array}\right]
$$


Lembrando serem simétricas as matrizes $\mathrm{S}_{\mathrm{II}}$ e $\mathrm{S}_{\mathrm{EE}}$.

$$
\begin{aligned}
& S_{I I(j, k)}^{*}=\sum_{i=1}^{N i} \sum_{j=i+1}^{N i} \sum_{k=j}^{N i} S_{I I(j, k)}-\frac{S_{I I(i, j)}}{S_{I I(i, i)}} S_{I I(i, k)} \\
& S_{I E(j, k)}^{*}=\sum_{i=1}^{N i} \sum_{j=i+1}^{N i} \sum_{k=N i}^{N i+N e} S_{I E(j, k-N i)}-\frac{S_{I I(i, j)}}{S_{I I(i, i)}} S_{I E(i, k-N i)} \\
& S_{E E(j, k)}^{*}=\sum_{i=1}^{N i} \sum_{j=N i+1}^{N i+N e} \sum_{k=j}^{N i+N e} S_{E E(j-N i, k-N i)}-\frac{S_{I E(i, j-N i)}}{S_{I I(i, i)}} S_{I E(i, k-N i)} \\
& F_{I(j)}^{*}=\sum_{i=1}^{N i} \sum_{j=i+1}^{N i} F_{I(j)}-\frac{S_{I I(i, j)}}{S_{I I(i, i)}} F_{I(i)} \\
& F_{E(j)}^{*}=\sum_{i=1}^{N i} \sum_{j=N i+1}^{N i+N e} F_{E(j-N i)}-\frac{S_{I E(i, j-N i)}}{S_{I I(i, i)}} F_{I(i)}
\end{aligned}
$$

ETAPA 2: Cálculo dos deslocamentos externos, resolvendo o sistema de equações:

$$
\mathrm{S}_{\mathrm{EE}}^{*} \mathrm{U}_{\mathrm{E}}=\mathrm{F}_{\mathrm{E}}^{*} \underset{\mathrm{ETAPA} 2}{\Rightarrow} \mathrm{U}_{\mathrm{E}}
$$

ETAPA 3: Cálculo dos deslocamentos internos, por retrosubstituição parcial a partir da última equação interna condensada:

$$
\begin{aligned}
& {\left[\begin{array}{cc}
\mathrm{S}_{\mathrm{II}}^{*} & \mathrm{~S}_{\mathrm{IE}}^{*} \\
0 & \mathrm{I}
\end{array}\right]\left[\begin{array}{c}
\mathrm{U}_{\mathrm{I}} \\
\mathrm{U}_{\mathrm{E}}
\end{array}\right]=\left[\begin{array}{c}
\mathrm{F}_{\mathrm{I}}^{*} \\
\mathrm{U}_{\mathrm{E}}
\end{array}\right] \underset{\text { ETAPA 3 }}{\Rightarrow}\left[\begin{array}{c}
\mathrm{U}_{\mathrm{I}} \\
\mathrm{U}_{\mathrm{E}}
\end{array}\right]} \\
& \mathrm{U}_{\mathrm{I}(\mathrm{j})}=\sum_{\mathrm{j}=\mathrm{Ni}}^{1} \frac{1}{\mathrm{~S}_{\mathrm{II}(\mathrm{j}, \mathrm{j})}^{*}}\left(\mathrm{~F}_{\mathrm{I}(\mathrm{j})}^{*}-\sum_{\mathrm{k}=\mathrm{Ni}+1}^{\mathrm{Ni}+\mathrm{Ne}} \mathrm{S}_{\mathrm{IE}(\mathrm{j}, \mathrm{k}-\mathrm{Ni})}^{*} \mathrm{U}_{\mathrm{E}(\mathrm{k}-\mathrm{Ni})}\right.
\end{aligned}
$$




\section{7 - Exemplo de aplicação}

Calcular os deslocamentos da estrutura plana abaixo (pórtico e treliça), utilizando subestruturação em paralelo. Há 2 nós em comum entre elas.
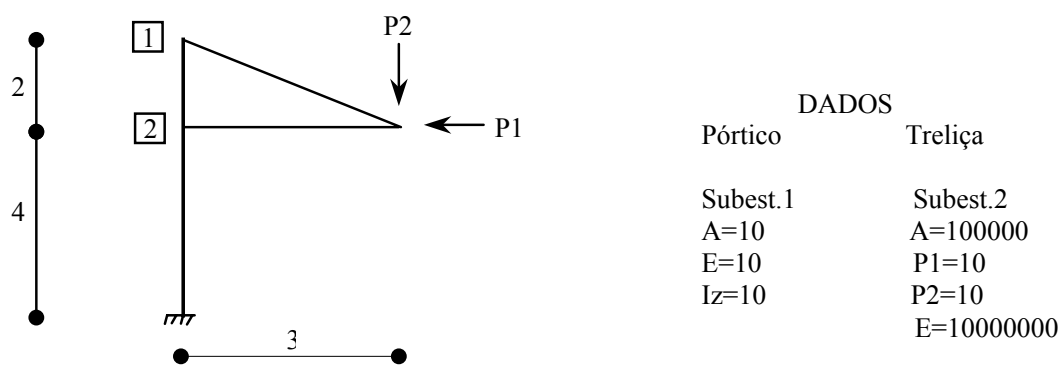

FIGURA 2.6 - Pilar e treliça

Formação das matrizes das subestruturas:

$$
\left[\begin{array}{ll}
\mathrm{S}_{\mathrm{II}}^{\mathrm{n}} & \mathrm{S}_{\mathrm{IE}}^{\mathrm{n}} \\
\mathrm{S}_{\mathrm{EI}}^{\mathrm{n}} & \mathrm{S}_{\mathrm{EE}}^{\mathrm{n}}
\end{array}\right]\left[\begin{array}{c}
\mathrm{U}_{\mathrm{I}}^{\mathrm{n}} \\
\mathrm{U}_{\mathrm{E}}^{\mathrm{n}}
\end{array}\right]=\left[\begin{array}{c}
\mathrm{F}_{\mathrm{I}}^{\mathrm{n}} \\
\mathrm{F}_{\mathrm{E}}^{\mathrm{n}}
\end{array}\right]
$$

Subestrutura 1 (Pilar):
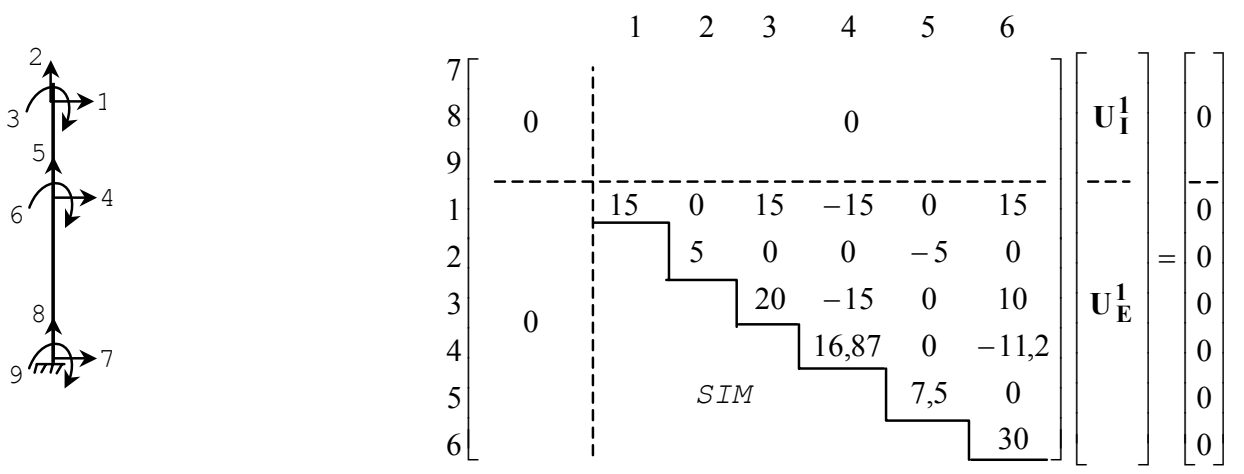

Subestrutura 2 (treliça) :
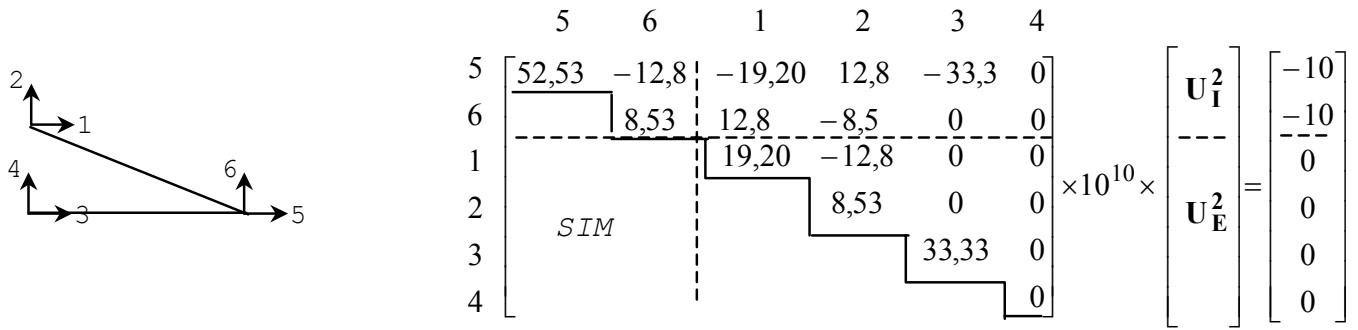

Impondo, nesta formação, as condições de vínculos verdadeiros e artificiais. 
Etapa 1:

$$
\left[\begin{array}{ll}
\mathrm{S}_{\mathrm{II}}^{\mathrm{n}} & \mathrm{S}_{\mathrm{IE}}^{\mathrm{n}} \\
\mathrm{S}_{\mathrm{EI}}^{\mathrm{n}} & \mathrm{S}_{\mathrm{EE}}^{\mathrm{n}}
\end{array}\right]\left[\begin{array}{c}
\mathrm{U}_{\mathrm{I}}^{\mathrm{n}} \\
\mathrm{U}_{\mathrm{E}}^{\mathrm{n}}
\end{array}\right]=\left[\begin{array}{c}
\mathrm{F}_{\mathrm{I}}^{\mathrm{n}} \\
\mathrm{F}_{\mathrm{E}}^{\mathrm{n}}
\end{array}\right] \Rightarrow\left[\begin{array}{cc}
\mathrm{S}_{\mathrm{II}}^{\mathrm{n}^{*}} & \mathrm{~S}_{\mathrm{IE}}^{\mathrm{n}^{*}} \\
0 & \mathrm{~S}_{\mathrm{EE}}^{\mathrm{n}^{*}}
\end{array}\right]\left[\begin{array}{c}
\mathrm{U}_{\mathrm{I}}^{\mathrm{n}} \\
\mathrm{U}_{\mathrm{E}}^{\mathrm{n}}
\end{array}\right]=\left[\begin{array}{l}
\mathrm{F}_{\mathrm{I}}^{\mathrm{n}^{*}} \\
\mathrm{~F}_{\mathrm{E}}^{\mathrm{n}^{*}}
\end{array}\right]
$$

Subestrutura 1 (pilar)
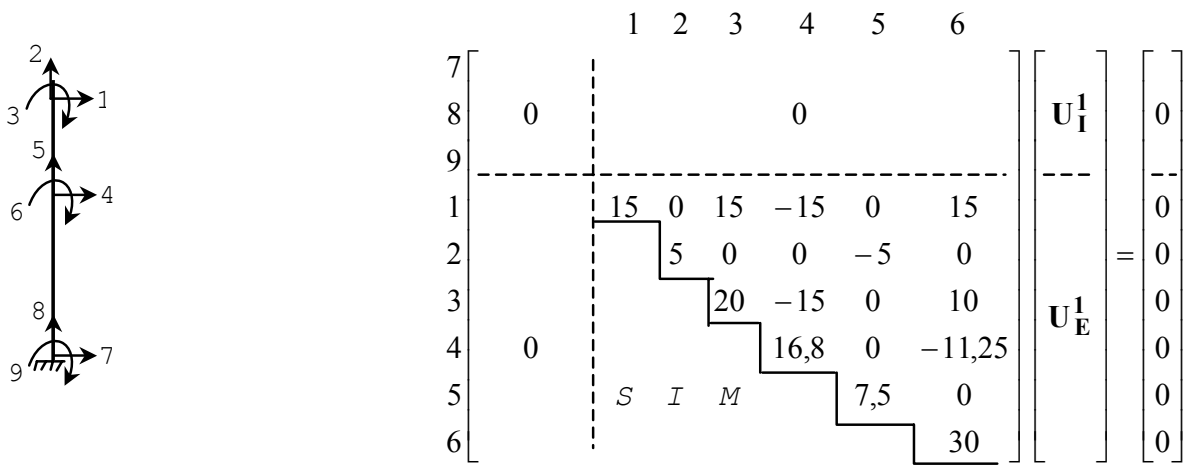

Subestrutura 2 (treliça) :
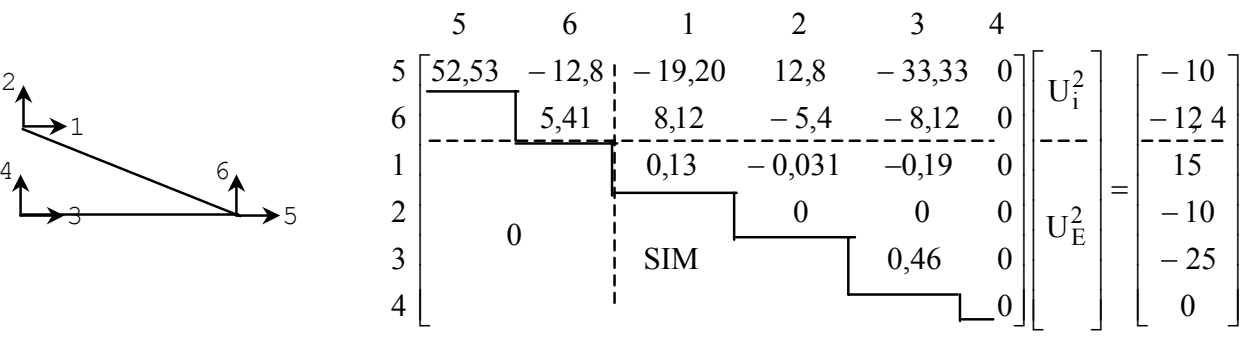

\section{Etapa 2:}

$$
\mathrm{S}_{\mathrm{C}}=\sum_{\mathrm{i}=1}^{2} \mathrm{~S}_{\mathrm{EEi}}^{*} \quad \mathrm{~F}_{\mathrm{C}}=\sum_{\mathrm{i}=1}^{2} \mathrm{~F}_{\mathrm{E}_{\mathrm{i}}}^{*} \quad \mathrm{~S}_{\mathrm{C}} \mathrm{U}_{\mathrm{C}}=\mathrm{F}_{\mathrm{C}}
$$

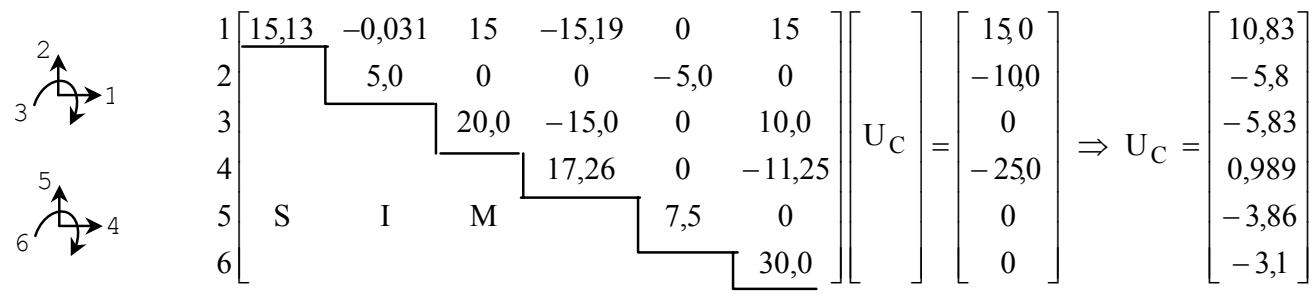

Formando $\mathrm{S}_{\mathrm{C}}$ com a somatória das coordenadas correspondentes entre as duas subestruturas 
Etapa 3:

$$
\left[\begin{array}{cc}
\mathrm{S}_{\mathrm{II}}^{\mathrm{i}^{*}} & \mathrm{~S}_{\mathrm{IE}}^{\mathrm{i}^{*}} \\
& \mathrm{I}^{\mathrm{i}}
\end{array}\right]\left[\begin{array}{c}
\mathrm{U}_{\mathrm{I}}^{\mathrm{i}} \\
\mathrm{U}_{\mathrm{E}}^{\mathrm{i}}
\end{array}\right]=\left[\begin{array}{c}
\mathrm{F}_{\mathrm{I}}^{\mathrm{i}^{*}} \\
\mathrm{U}_{\mathrm{E}}^{\mathrm{i}}
\end{array}\right] \Rightarrow\left[\begin{array}{c}
\mathrm{U}_{\mathrm{I}}^{\mathrm{i}} \\
\mathrm{U}_{\mathrm{E}}^{\mathrm{i}}
\end{array}\right]
$$

Subestrutura 1 (pilar):

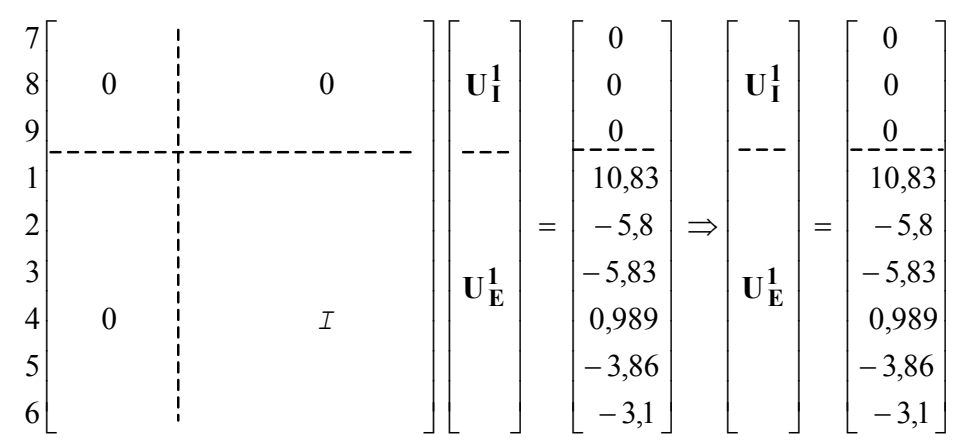

Subestrutura 2 (treliça):

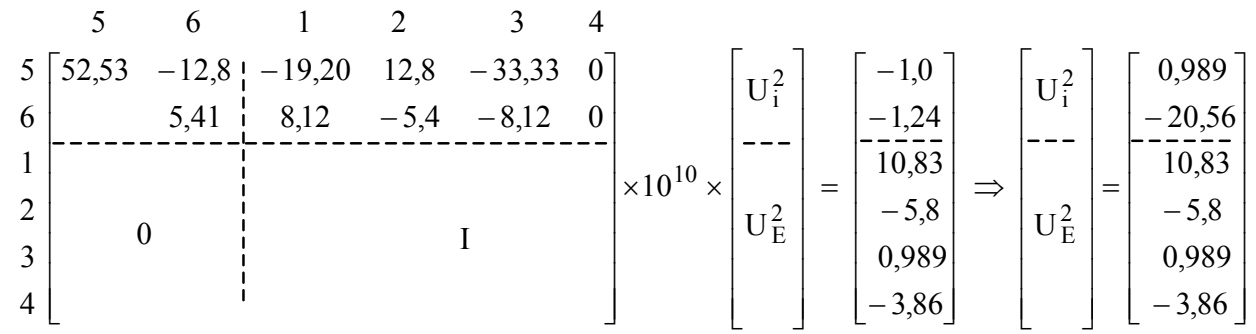

Após a determinação de todos os deslocamentos nodais, internos e externos, poderá se proceder à análise completa de cada subestrutura individualmente, obtendo-se os esforços de extremidade dos elementos e as reações de apoio. 


\title{
CAPÍTULO 3 - IMPLEMENTAÇÃO DO GERADOR GRÁFICO
}

\author{
Será considerado como sistema estrutural a estutura \\ tridimensional formada por subestruturas tridimensionais \\ e também por aquelas que se limitam a um plano. \\ - programa desenvolvido conterá subrotinas para o \\ tratamento dos tipos principais de subestruturas, \\ aporticadas e treliçadas. Irá permitir a compatibilização \\ dos deslocamentos comuns entre elas, permitindo a análise \\ da grande maioria dos sistemas estruturais formados por \\ barras.
}

\section{1 - Sistema de referência global}

O sistema estrutural terá como referência os eixos cartesianos X, Y e Z, com origem "O" situada num ponto qualquer, definido pelo usuário. As subestruturas formadas também serão referenciadas por este sistema. Todas as transformações, então, serão efetuadas com base neste sistema também. 


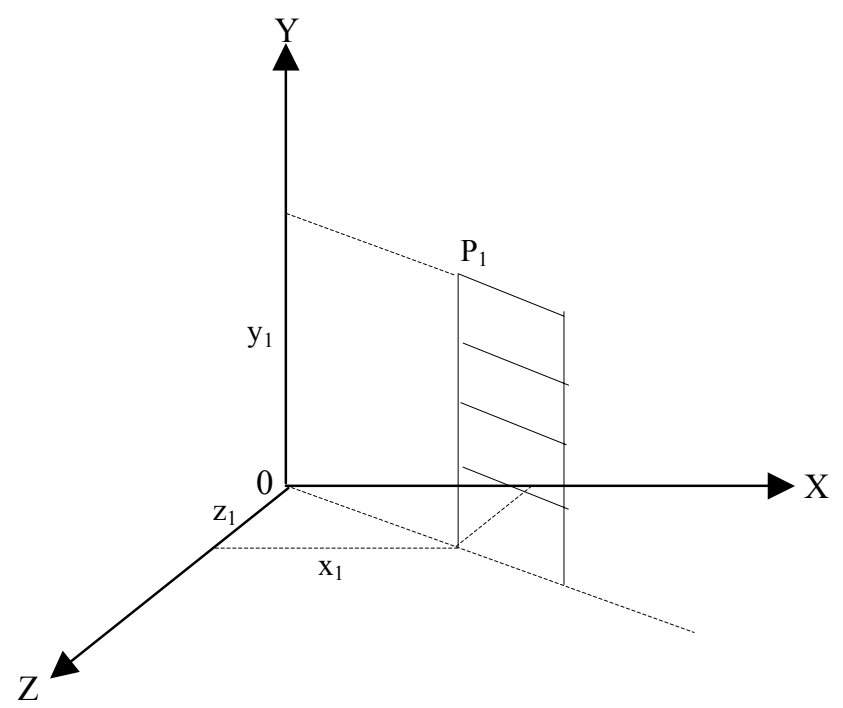

FIGURA 3.1 - Sistema de referência global

\section{2 - Características das subestruturas.}

Serão abordados alguns tópicos importantes na formulação matricial do método dos deslocamentos, adotados neste programa, e que envolvem a formação das matrizes de rigidez e de forças dos elementos estruturais.

Também será demonstrado a maneira como foi idealizada a transformação destas grandezas, do sistema de coordenadas locais para o sistema de coordenadas da estrutura global, atravéz das matrizes de rotação. 


\subsection{1 - Treliça Espacial}

Na Análise de uma treliça espacial, são tomadas em consideração as deformações axiais, com a possibilidade de três deslocamentos independentes em cada nó.

A FIGURA 3.2 mostra um elemento desta classe de estrutura, com seus nós, inicial e final $(j, k)$, e a numeração de suas coordenadas de membro.

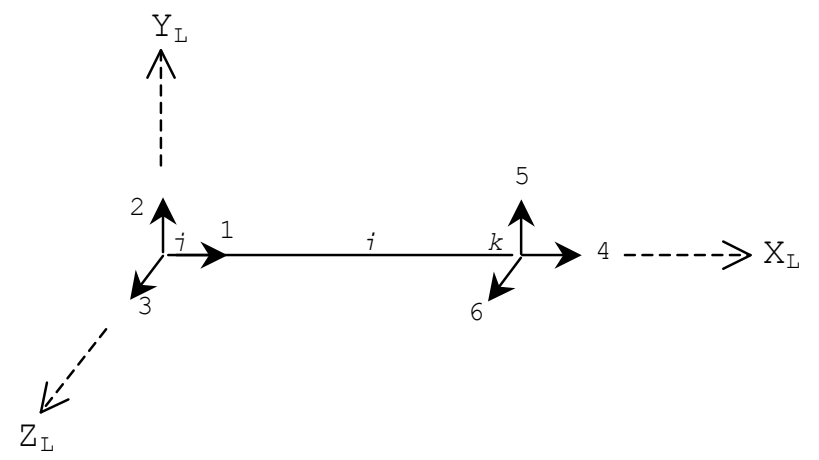

FIGURA 3.2 - Elemento de treliça espacial que se desenvolve pelo eixo $\mathrm{X}_{\mathrm{L}}$

A matriz de rigidez do elemento em relação aos eixos de membro é:

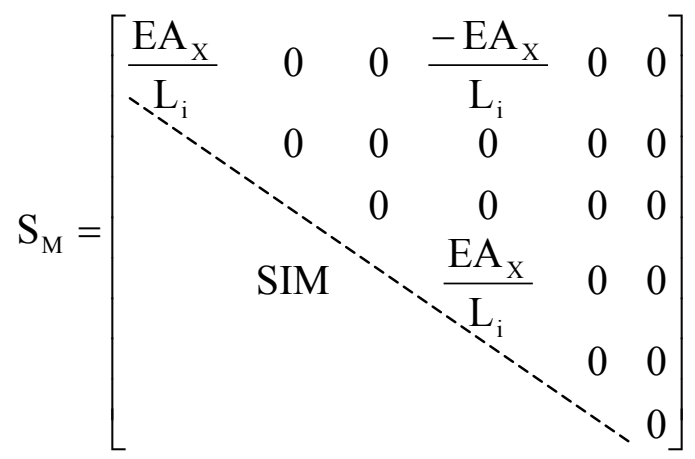

A matriz de rotação $R_{\text {ot }}$ será determinada e utilizada para a transformação de ações e deslocamentos dos eixos de membro para os eixos da estrutura, conforme FIGURA 3.3 


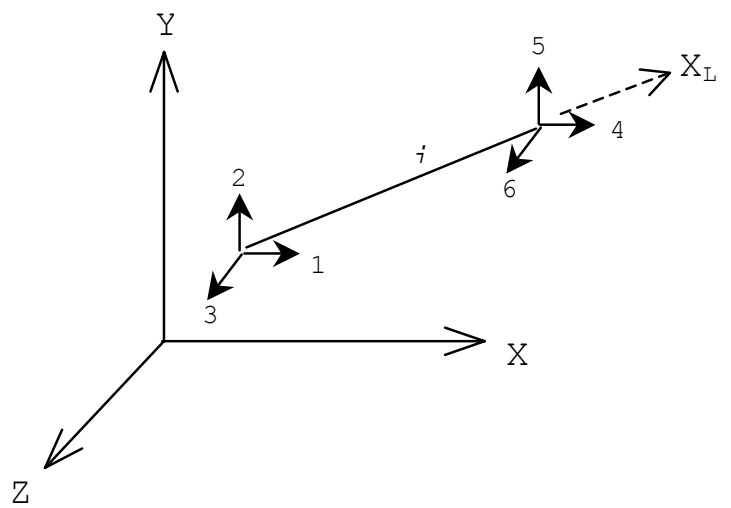

FIGURA 3.3 - Ações no elemento i em relação aos eixos do sistema estrutural (X,Y,Z).

No tratamento de ações e deslocamentos que atuam sobre o elemento, é necessário selecionar uma orientação específica para os três eixos de membro $\left(\mathrm{X}_{\mathrm{L}}, \mathrm{Y}_{\mathrm{L}}, \mathrm{Z}_{\mathrm{L}}\right)$, em relação ao sistema global.

Como o eixo $\mathrm{X}_{\mathrm{L}}$ já foi definido como eixo da barra, resta definir as direções dos restantes. O elemento típico i é novamente visualizado (FIGURA 3.4):

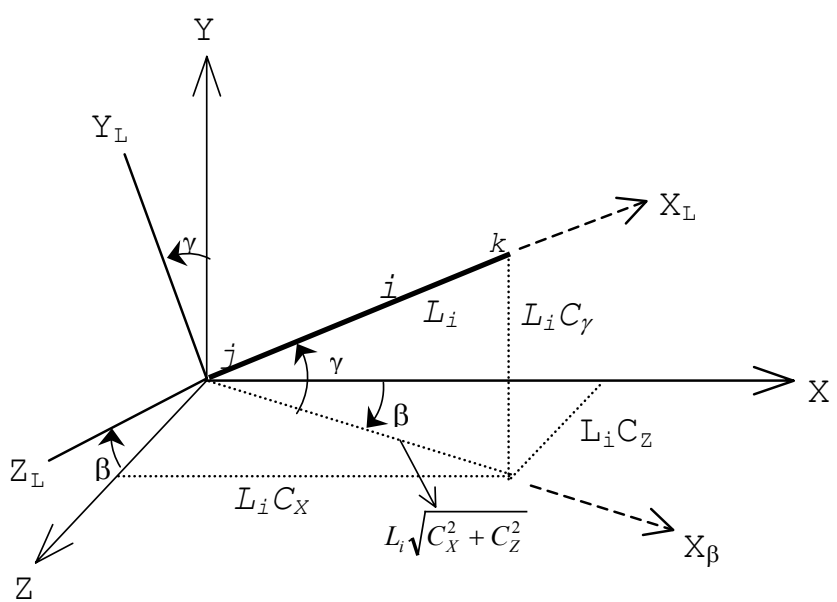

FIGURA 3.4 - Relação entre os eixos locais e os do sistema estrutural. 
Sendo:

$$
\mathrm{L}_{\mathrm{i}}=\sqrt{\left(\mathrm{x}_{\mathrm{k}}-\mathrm{x}_{\mathrm{j}}\right)^{2}+\left(\mathrm{y}_{\mathrm{k}}-\mathrm{y}_{\mathrm{j}}\right)^{2}+\left(\mathrm{z}_{\mathrm{k}}-\mathrm{z}_{\mathrm{j}}\right)^{2}}
$$

E os co-senos diretores:

$$
\mathrm{C}_{\mathrm{x}}=\frac{\mathrm{x}_{\mathrm{k}}-\mathrm{x}_{\mathrm{j}}}{\mathrm{L}_{\mathrm{i}}} \quad \mathrm{C}_{\mathrm{y}}=\frac{\mathrm{y}_{\mathrm{k}}-\mathrm{y}_{\mathrm{j}}}{\mathrm{L}_{\mathrm{i}}} \quad \mathrm{C}_{\mathrm{z}}=\frac{\mathrm{z}_{\mathrm{k}}-\mathrm{z}_{\mathrm{j}}}{\mathrm{L}_{\mathrm{i}}}
$$

Por conveniência, torna-se 0 eixo $Z_{L}$ como sendo horizontal (no plano $\mathrm{X}-\mathrm{Z}$ ), então $O$ eixo $\mathrm{Y}_{\mathrm{L}}$ estará num plano vertical que passa pelos eixos $X_{L}$ e $Y$.

Adota-se que $Z_{L}$ estará num plano horizontal acompanhando o eixo Z, conforme FIGURA 3.5, que visualiza duas possibilidades.
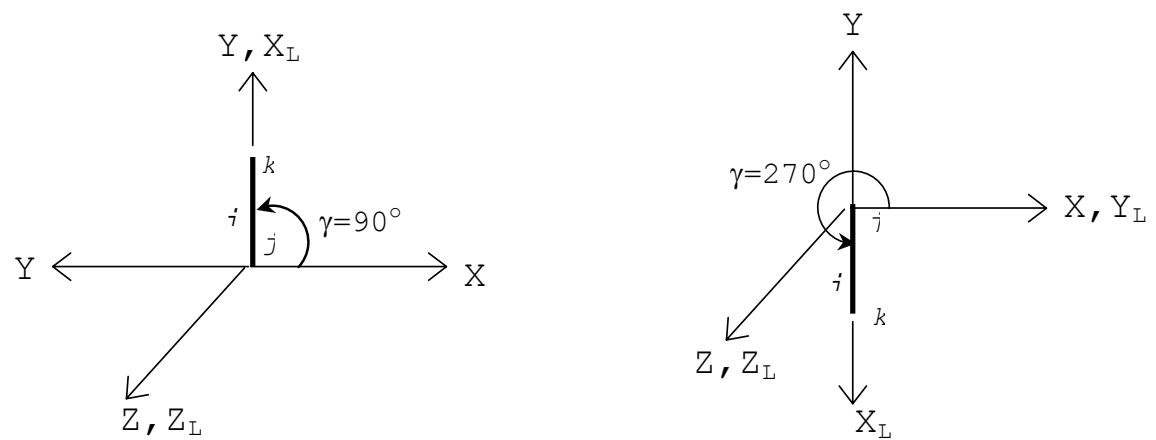

FIGURA 3.5 - Elemento vertical

A aplicação das transformações necessárias compõe-se por rotações sucessivas de eixos, tomada em duas etapas.

A primeira é um giro de angulo $\beta$ em torno de $Y_{L}$, colocando o eixo $X$ na posição $x_{\beta}$, que é a inclinação do plano $X-Z$ com $O$ plano $X_{L}-Y$. O eixo $Z_{L}$ também toma sua posição final, com angulo $\beta$ em relação a $Z$.

Esta rotação se dá: 


$$
\mathrm{R}_{\beta}=\left[\begin{array}{ccc}
\cos \beta & 0 & \operatorname{sen} \beta \\
0 & 1 & 0 \\
-\operatorname{sen} \beta & 0 & \cos \beta
\end{array}\right] \quad \operatorname{com} \quad \begin{aligned}
& \cos \beta=\frac{C_{x}}{\sqrt{C_{x}^{2}+C_{z}^{2}}} \\
& \operatorname{sen} \beta=\frac{C_{z}}{\sqrt{C_{x}^{2}+C_{z}^{2}}}
\end{aligned}
$$

Então:

$$
\mathrm{R}_{\beta}=\left[\begin{array}{ccc}
\frac{\mathrm{C}_{\mathrm{x}}}{\sqrt{\mathrm{C}_{\mathrm{x}}^{2}+\mathrm{C}_{\mathrm{z}}^{2}}} & 0 & \frac{\mathrm{C}_{\mathrm{z}}}{\sqrt{\mathrm{C}_{\mathrm{x}}^{2}+\mathrm{C}_{\mathrm{z}}^{2}}} \\
0 & 1 & 0 \\
-\frac{\mathrm{C}_{\mathrm{z}}}{\sqrt{\mathrm{C}_{\mathrm{x}}^{2}+\mathrm{C}_{\mathrm{z}}^{2}}} & 0 & \frac{\mathrm{C}_{\mathrm{x}}}{\sqrt{\mathrm{C}_{\mathrm{x}}^{2}+\mathrm{C}_{\mathrm{z}}^{2}}}
\end{array}\right]
$$

A Segunda etapa consiste em um giro de um angulo $\gamma$ em torno do eixo $Z_{L}$, colocando os eixos $X_{L} e Y_{L}$ em suas posições finais.

Sendo:

$$
\mathrm{R}_{\gamma}=\left[\begin{array}{ccc}
\cos \gamma & \operatorname{sen} \gamma & 0 \\
-\operatorname{sen} \gamma & \cos \gamma & 0 \\
0 & 0 & 1
\end{array}\right] \quad \operatorname{com} \quad \begin{gathered}
\cos \gamma=\sqrt{\mathrm{C}_{\mathrm{x}}^{2}+\mathrm{C}_{\mathrm{z}}^{2}} \\
\operatorname{sen} \gamma=\mathrm{C}_{\mathrm{y}}
\end{gathered}
$$

Então:

$$
R_{\gamma}=\left[\begin{array}{ccc}
\sqrt{C_{x}^{2}+C_{z}^{2}} & C_{y} & 0 \\
-C_{y} & \sqrt{C_{x}^{2}+C_{z}^{2}} & 0 \\
0 & 0 & 1
\end{array}\right]
$$

Os dois giros, em separado, são componentes de uma transformação completa dos eixos da estrutura, representada pela matriz $\mathrm{R}_{\text {ot }}$. Ou seja: 


$$
R_{o t}=\left[\begin{array}{ccc}
C_{x} & C_{y} & C_{z} \\
-\frac{C_{x} C_{y}}{\sqrt{C_{x}^{2}+C_{z}^{2}}} & \sqrt{C_{x}^{2}+C_{z}^{2}} & -\frac{C_{y} C_{z}}{\sqrt{C_{x}^{2}+C_{z}^{2}}} \\
-\frac{C_{z}}{\sqrt{C_{x}^{2}+C_{z}^{2}}} & 0 & \frac{C_{x}}{\sqrt{C_{x}^{2}+C_{z}^{2}}}
\end{array}\right]
$$

Quando o elemento é vertical, $X_{L}$ paralelo a Y, a matriz $\mathrm{R}_{\mathrm{ot}}$ passa a ser:

$$
\mathrm{R}_{\mathrm{ot}}=\left[\begin{array}{ccc}
0 & \mathrm{C}_{\mathrm{y}} & 0 \\
-\mathrm{C}_{\mathrm{y}} & 0 & 0 \\
0 & 0 & 1
\end{array}\right]
$$

Uma matriz de rigidez da barra $\mathrm{S}_{\mathrm{m}}$ contém elementos que relacionam duas grandezas físicas, força e deslocamento, e quando transformada para um sistema global de coordenadas toma a seguinte forma:

$$
\mathrm{S}_{\mathrm{MD}}=\mathrm{R}_{\mathrm{ot}}^{\mathrm{T}} \mathrm{S}_{\mathrm{m}} \mathrm{R}_{\mathrm{ot}}
$$

Logo:

$$
\mathrm{S}_{\mathrm{MD}}=\frac{\mathrm{EA}_{\mathrm{x}}}{\mathrm{L}_{\mathrm{i}}}\left[\begin{array}{cccccc}
\mathrm{C}_{\mathrm{x}}^{2} & \mathrm{C}_{\mathrm{y}} \mathrm{C}_{\mathrm{x}} & \mathrm{C}_{\mathrm{z}} \mathrm{C}_{\mathrm{x}} & -\mathrm{C}_{\mathrm{x}}^{2} & -\mathrm{C}_{\mathrm{y}} \mathrm{C}_{\mathrm{x}} & -\mathrm{C}_{\mathrm{z}} \mathrm{C}_{\mathrm{x}} \\
\mathrm{C}_{\mathrm{x}} \mathrm{C}_{\mathrm{y}} & \mathrm{C}_{\mathrm{y}}^{2} & \mathrm{C}_{\mathrm{z}} \mathrm{C}_{\mathrm{y}} & -\mathrm{C}_{\mathrm{x}} \mathrm{C}_{\mathrm{y}} & -\mathrm{C}_{\mathrm{y}}^{2} & -\mathrm{C}_{\mathrm{z}} \mathrm{C}_{\mathrm{y}} \\
\mathrm{C}_{\mathrm{x}} \mathrm{C}_{\mathrm{z}} & \mathrm{C}_{\mathrm{y}} \mathrm{C}_{\mathrm{z}} & \mathrm{C}_{\mathrm{z}}^{2} & -\mathrm{C}_{\mathrm{x}} \mathrm{C}_{\mathrm{z}} & -\mathrm{C}_{\mathrm{y}} \mathrm{C}_{\mathrm{z}} & \mathrm{C}_{\mathrm{z}}^{2} \\
-\mathrm{C}_{\mathrm{x}}^{2} & -\mathrm{C}_{\mathrm{y}} \mathrm{C}_{\mathrm{x}} & -\mathrm{C}_{\mathrm{z}} \mathrm{C}_{\mathrm{x}} & -\mathrm{C}_{\mathrm{x}}^{2} & \mathrm{C}_{\mathrm{y}} \mathrm{C}_{\mathrm{x}} & \mathrm{C}_{\mathrm{z}} \mathrm{C}_{\mathrm{x}} \\
-\mathrm{C}_{\mathrm{x}} \mathrm{C}_{\mathrm{y}} & -\mathrm{C}_{\mathrm{y}}^{2} & -\mathrm{C}_{\mathrm{z}} \mathrm{C}_{\mathrm{y}} & \mathrm{C}_{\mathrm{x}} \mathrm{C}_{\mathrm{y}} & \mathrm{C}_{\mathrm{y}}^{2} & \mathrm{C}_{\mathrm{z}} \mathrm{C}_{\mathrm{y}} \\
-\mathrm{C}_{\mathrm{x}} \mathrm{C}_{\mathrm{z}} & -\mathrm{C}_{\mathrm{y}} \mathrm{C}_{\mathrm{z}} & -\mathrm{C}_{\mathrm{z}}^{2} & \mathrm{C}_{\mathrm{x}} \mathrm{C}_{\mathrm{z}} & \mathrm{C}_{\mathrm{y}} \mathrm{C}_{\mathrm{z}} & \mathrm{C}_{\mathrm{z}}^{2}
\end{array}\right]
$$


Para este tipo de estrutura, o carregamento será aplicado nos nós, diretamente relacionados ao sistema da estrutura, formando o vetor $\mathrm{F}$ de cargas nodais.

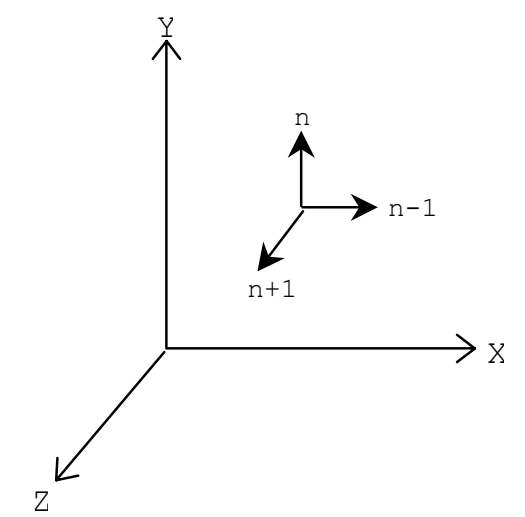

$$
\mathrm{F}=\left[\begin{array}{c}
\cdot \\
\cdot \\
\mathrm{n}-1 \\
\mathrm{n} \\
\mathrm{n}+1 \\
\cdot \\
\cdot
\end{array}\right]
$$

FIGURA 3.6 - Forças aplicadas em nós de treliça

\subsection{2 - Pórtico espacial}

Na análise de um pórtico espacial, são considerados 6 possíveis deslocamentos independentes em cada nó.

A FIGURA 3.7 mostra um elemento deste tipo estrutural, orientado segundo seu sistema de coordenadas de barras, com a numeração dos 12 deslocamentos possíveis:

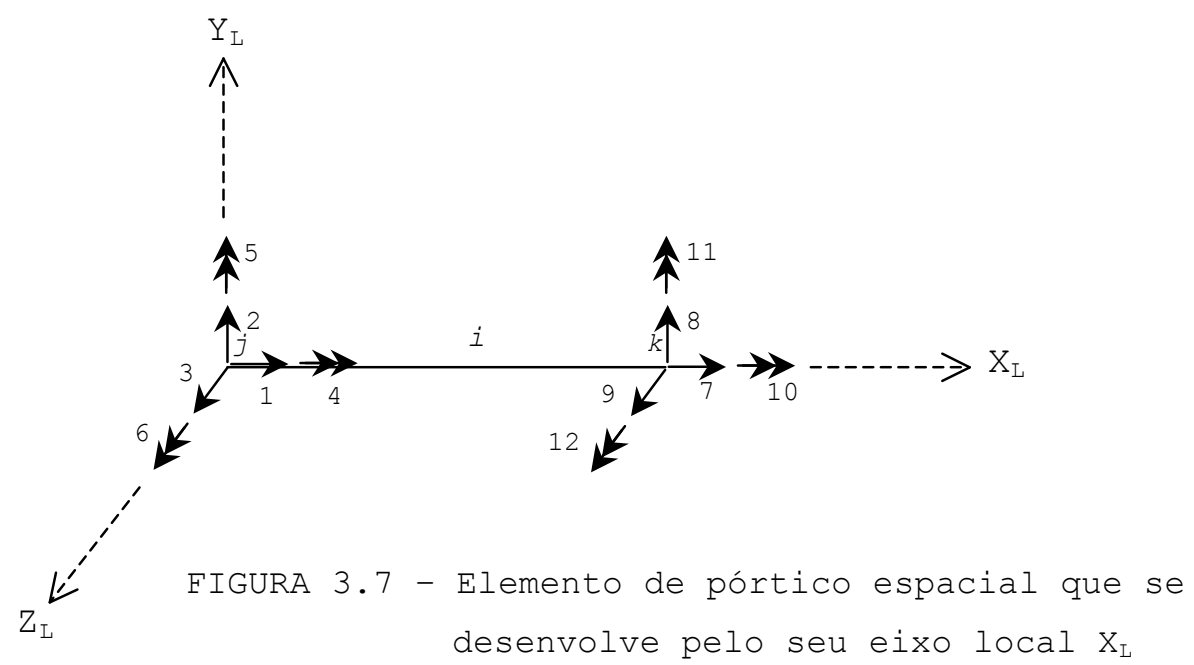


A matriz de rigidez, em relação ao eixo da barra é:

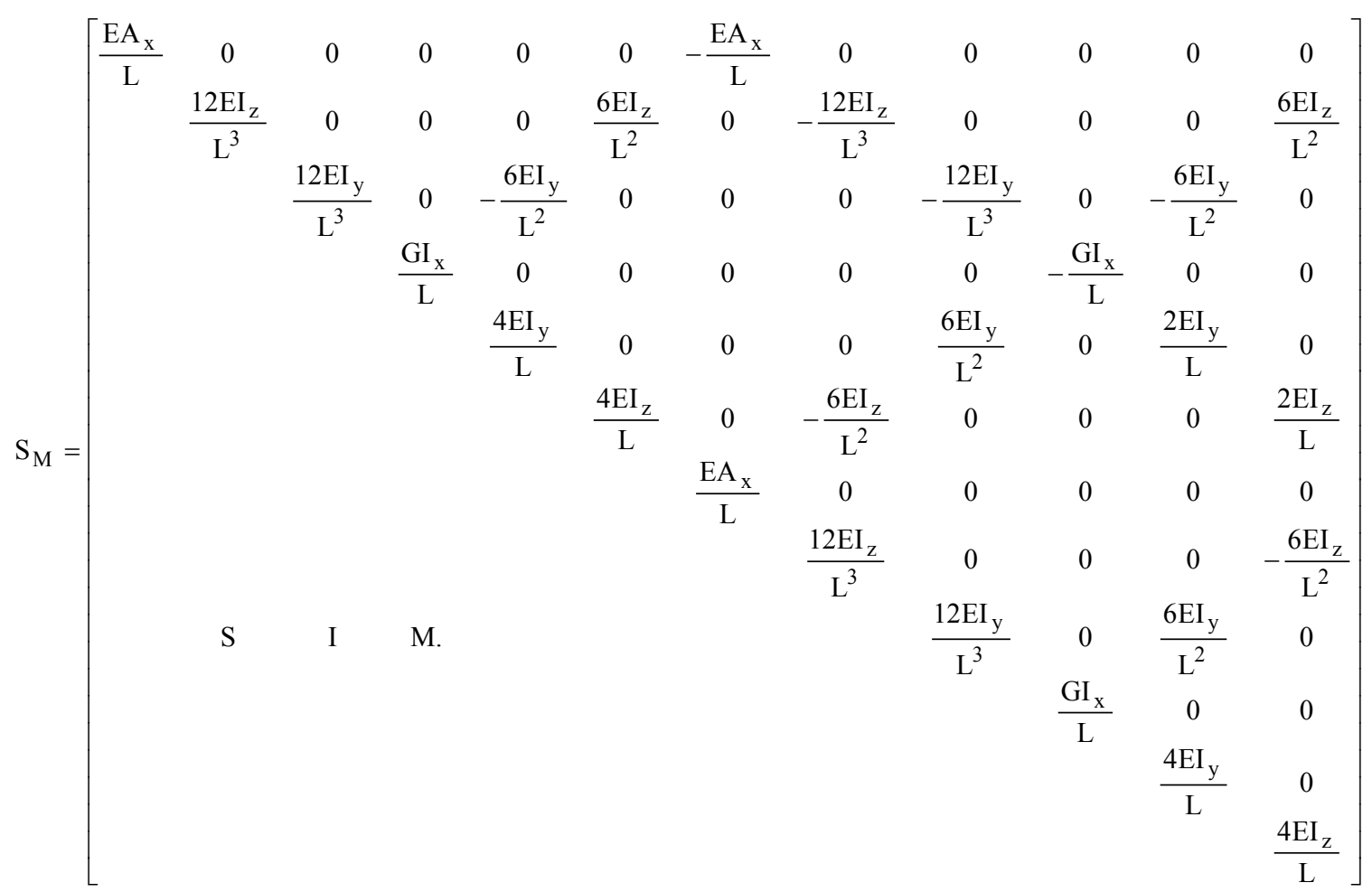

A rotação das grandezas, que envolve um elemento de pórtico tridimensional, do sistema de coordenadas local para o sistema global, é uma continuidade ao processo anterior para treliças.

Um membro de um pórtico será orientado para que seus eixos principais da seção transversal existam nos planos, horizontal e vertical. Nesta situação, os eixos $Y_{L}$ e $Z_{I}$ são escolhidos de forma semelhante a um membro de treliça.

Em outras vezes, o elemento de pórtico pode ter seus eixos principais $Y_{L}$ e $Z_{L}$ em direções oblíquas, com um giro de ângulo $\alpha$ em torno do eixo da barra $\mathrm{X}_{\mathrm{L}}$, conforme FIGURA 3.8 . 

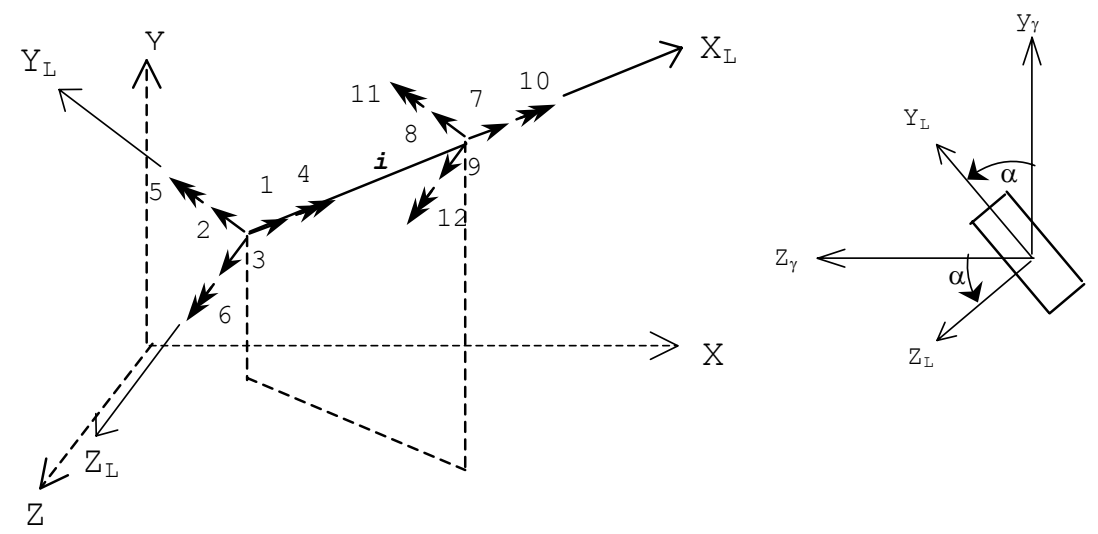

$$
\begin{aligned}
\text { FIGURA3.8 - } & \text { Relação entre eixos locais e eixos da } \\
& \text { estrutura, incluindo angulo } \alpha \text { de rotação }
\end{aligned}
$$
da seção transversal em torno de $\mathrm{X}_{\mathrm{L}}$

A transformação de coordenadas deste elemento consistirá em três rotações, acompanhadas a partir da FIGURA 3.9 :

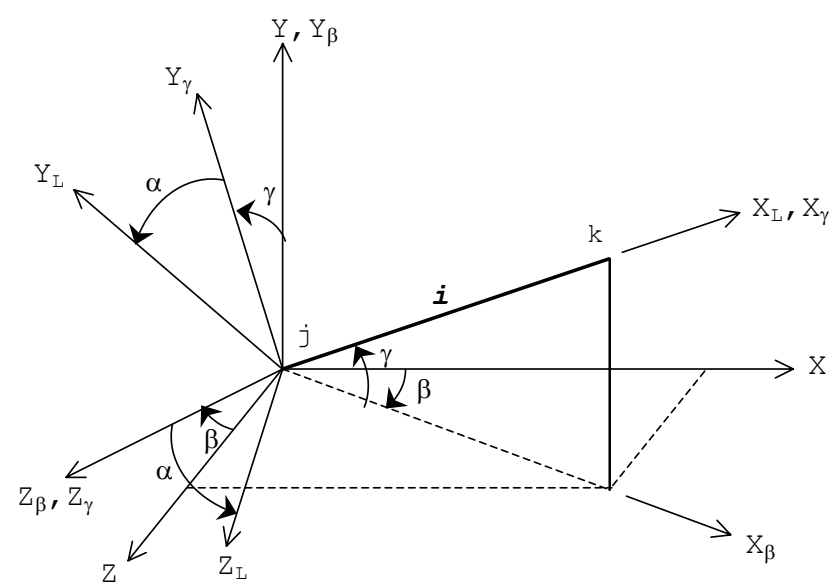

FIGURA 3.9 - Relaçao entre eixos locais e eixos da estrutura para elemento de pórtico espacial 
As duas primeiras rotações de ângulos $\beta$ e $\gamma$, em torno de $Y$ e $Z_{\beta}$, são as mesmas que da FIGURA 3.4 para treliça.

A terceira transformação consiste numa rotação do angulo $\alpha$ em torno de $\mathrm{X}_{\mathrm{L}}$, fazendo que os eixos $\mathrm{Y}_{\mathrm{L}}$ e $\mathrm{Z}_{\mathrm{L}}$ coincidam com os eixos principais da seção transversal.

O plano $\mathrm{X}_{\mathrm{L}}$ e $\mathrm{Y}_{\gamma}$ é vertical e que passa pelo eixo da barra, e o angulo $\alpha$ é medido deste plano a um dos eixos principais da seção transversal. Requer uma matriz de rotação $R_{\alpha}$, com seus elementos sendo co-senos diretores dos eixos locais $\left(X_{L}, Y_{L}, Z_{L}\right)$ em relação aos eixos $X_{\gamma}, Y_{\gamma}, Z_{\gamma}$.

Esta matriz será:

$$
\mathrm{R}_{\alpha}=\left[\begin{array}{ccc}
1 & 0 & 0 \\
0 & \cos \alpha & \operatorname{sen} \alpha \\
0 & -\operatorname{sen} \alpha & \cos \alpha
\end{array}\right]
$$

A matriz de rotação para elementos de pórtico espacial será então:

$$
\mathrm{R}_{\mathrm{ot}}=\mathrm{R}_{\alpha} \mathrm{R}_{\gamma} \mathrm{R}_{\beta}
$$

Lembrando que as matrizes $\mathrm{R}_{\gamma}$ e $\mathrm{R}_{\beta}$ foram discutidas anteriormente, em rotação para barras de treliça.

A matriz resultante será:

$$
R_{o t}=\left[\begin{array}{ccc}
C_{x} & C_{y} & C_{z} \\
\frac{-C_{x} C_{y} \cos \alpha-C_{z} \operatorname{sen} \alpha}{\sqrt{C_{x}^{2}+C_{z}^{2}}} & \sqrt{C_{x}^{2}+C_{z}^{2}} \cos \alpha & \frac{-C_{y} C_{z} \cos \alpha+C_{x} \operatorname{sen} \alpha}{\sqrt{C_{x}^{2}+C_{z}^{2}}} \\
\frac{C_{x} C_{y} \operatorname{sen} \alpha-C_{z} \cos \alpha}{\sqrt{C_{x}^{2}+C_{z}^{2}}} & -\sqrt{C_{x}^{2}+C_{z}^{2}} \operatorname{sen} \alpha & \frac{C_{y} C_{z} \operatorname{sen} \alpha+C_{x} \cos \alpha}{\sqrt{C_{x}^{2}+C_{z}^{2}}}
\end{array}\right]
$$


Quando o ângulo $\alpha$ é igual a zero, a matriz $R_{o t}$ se reduz à forma anterior, dada para barra de treliça espacial.

De uma maneira sucinta, quando o elemento estrutural é vertical, temos então:

$$
R_{o t}=\left[\begin{array}{ccc}
0 & C_{y} & 0 \\
-C_{y} \cos \alpha & 0 & \operatorname{sen} \alpha \\
C_{y} \operatorname{sen} \alpha & 0 & \cos \alpha
\end{array}\right]
$$

Da mesma forma, quando $\alpha$ é igual a zero, a matriz de rotação se reduz à forma anterior, para treliças.

A rotação da matriz de rigidez se dará por:

$$
\mathrm{S}_{\mathrm{MD}}=\mathrm{R}_{\mathrm{ot}}^{\mathrm{T}} \mathrm{SR}_{\mathrm{ot}}
$$

Os carregamentos impostos serão aplicados em duas formas distintas, ou seja:

Aplicados diretamente nos nós, relacionados ao sistema da estrutura.
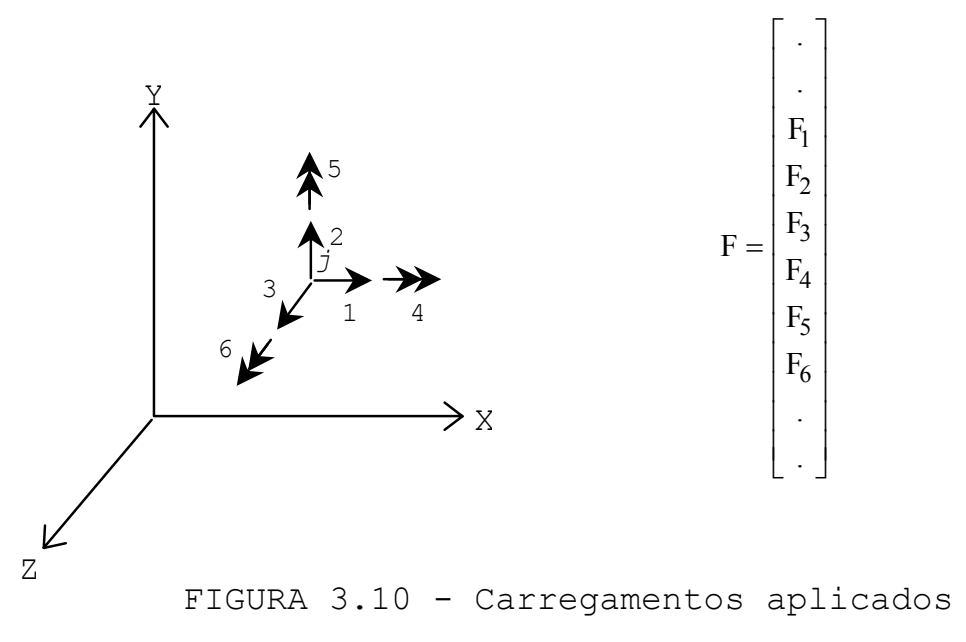
diretamente no nó j. 
Os carregamentos aplicados às barras da estrutura, referenciados pelo sistema local de coordenadas, serão transformados em cargas nodais equivalentes, e que estarão contidas no vetor $\mathbf{f}$.

Em cada elemento estrutural, definido por 2 nós, ocorrem 12 possíveis ações, dependendo do tipo e direção do carregamento aplicado.

Para um carregamento no plano $\mathrm{X}_{\mathrm{L}}-\mathrm{Y}_{\mathrm{L}}$ :

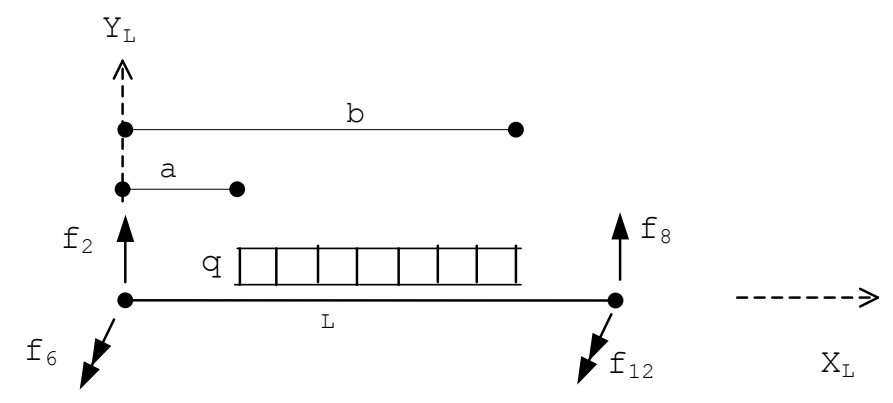

FIGURA 3.11 - Carregamento no plano $\mathrm{X}_{\mathrm{L}}-\mathrm{Y}_{\mathrm{L}}$

Com:

$$
\begin{aligned}
& \mathrm{a} 1=\mathrm{b}-\mathrm{a} \quad \mathrm{a} 2=(\mathrm{a}+\mathrm{b}) / 2 \quad \mathrm{a} 3=\mathrm{L}-\mathrm{a} 2 \quad \mathrm{a} 4=12 \sqrt{\mathrm{L}_{\mathrm{i}}} \\
& \begin{aligned}
\mathrm{L}_{\mathrm{i}}= & \text { comprimento da barra } \\
\mathrm{f} 2 & =(\mathrm{q} \cdot \mathrm{a} 1 \cdot \mathrm{a} 3+\mathrm{f} 6+\mathrm{f} 12) / \mathrm{L}_{\mathrm{i}} \\
\mathrm{f} 6 & =\text { q.a1. }\left((12 \cdot \mathrm{a} 2 \cdot \sqrt{\mathrm{a} 3})+\sqrt{\mathrm{a} 1} \cdot\left(\mathrm{L}_{\mathrm{i}}-3 \cdot \mathrm{a} 2\right)\right) / \mathrm{a} 4 \\
& \mathrm{f} 8=(\mathrm{q} \cdot \mathrm{a} 1 \cdot \mathrm{a} 2-\mathrm{f} 6-\mathrm{f} 12) / \mathrm{L}_{\mathrm{i}} \\
& \mathrm{f} 12=- \text { q.a1. }\left((12 \cdot \mathrm{a} 3 \cdot \sqrt{\mathrm{a} 2})+\sqrt{\mathrm{a} 1} \cdot\left(\mathrm{L}_{\mathrm{i}}-3 \cdot \mathrm{a} 2\right)\right) / \mathrm{a} 4
\end{aligned}
\end{aligned}
$$


O elemento acima, sujeito ao mesmo carregamento direcionado no plano $\mathrm{X}_{\mathrm{L}}-\mathrm{Z}_{\mathrm{L}}$ :

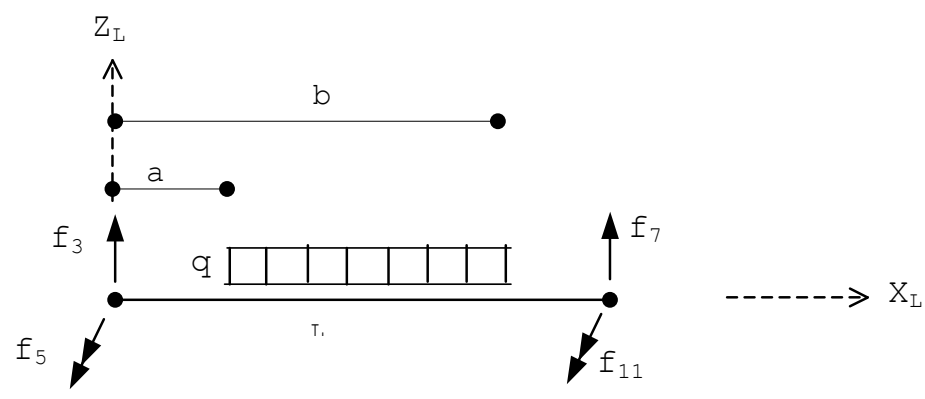

FIGURA 3.12 - Carregamento no plano $\mathrm{X}_{\mathrm{L}}-\mathrm{Z}_{\mathrm{I}}$

$$
\begin{aligned}
& \mathrm{f} 3=(\mathrm{q} \cdot \mathrm{a} 1 \cdot \mathrm{a} 3+\mathrm{f} 6+\mathrm{f} 12) / \mathrm{L}_{\mathrm{i}} \\
& \mathrm{f} 5=\mathrm{q} \cdot \mathrm{a} 1 \cdot\left((12 \cdot \mathrm{a} 2 \cdot \sqrt{\mathrm{a} 3})+\sqrt{\mathrm{a} 1} \cdot\left(\mathrm{L}_{\mathrm{i}}-3 \cdot \mathrm{a} 2\right)\right) / \mathrm{a} 4 \\
& \mathrm{f} 7=(\mathrm{q} \cdot \mathrm{a} 1 \cdot \mathrm{a} 2-\mathrm{f} 6-\mathrm{f} 12) / \mathrm{L}_{\mathrm{i}} \\
& \mathrm{f} 11=-\mathrm{q} \cdot \mathrm{a} 1 \cdot\left((12 \cdot \mathrm{a} 3 \cdot \sqrt{\mathrm{a} 2})+\sqrt{\mathrm{a} 1} \cdot\left(\mathrm{L}_{\mathrm{i}}-3 \cdot \mathrm{a} 2\right)\right) / \mathrm{a} 4
\end{aligned}
$$

Estas forças nodais são rotacionadas para os eixos da estrutura através de:

$$
\mathrm{f}_{\mathrm{R}}=\mathrm{R}_{\mathrm{ot}} \mathrm{f}
$$

E finalmente acopladas em $F$, que é o vetor de forças da estrutura, em relação aos eixos globais. 


\section{3 - Formação das subestruturas}

Dado um sistema estrutural, a sua divisão em subestruturas será condicionada aos fatores:

- Tipos estruturais: Quando um elemento de um tipo estrutural se une a outro, diferente, cada um pertencerá a subestruturas distintas.

- Quando uma subestrutura se torna muito grande, ou quando for conveniente, será dividida em outras subestruturas, unidas por elementos de mesmo tipo estrutural.

Cada subestrutura será discretizada individualmente, como se fosse uma única estrutura existente. Considere a figura 3.13, união de pilares (pórtico) com treliças:

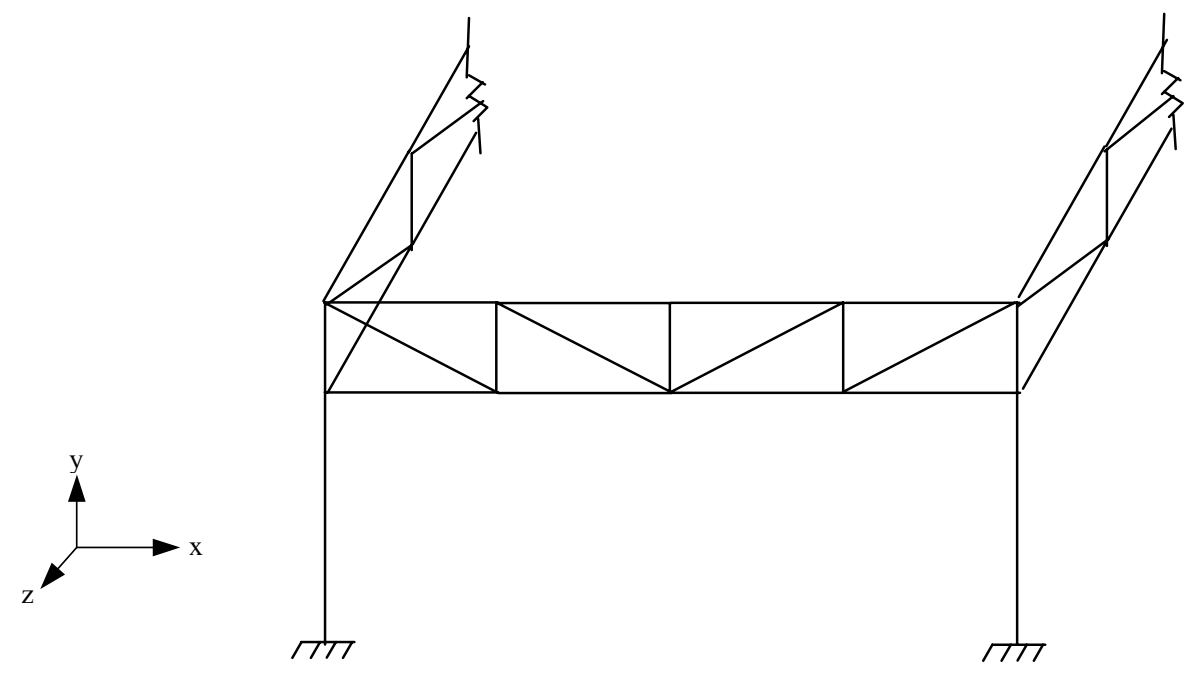

FIGURA 3.13 - Sistema estrutural com pórtico e treliça 
Separando em subestruturas, P1, P2, T1, T2 e T3:

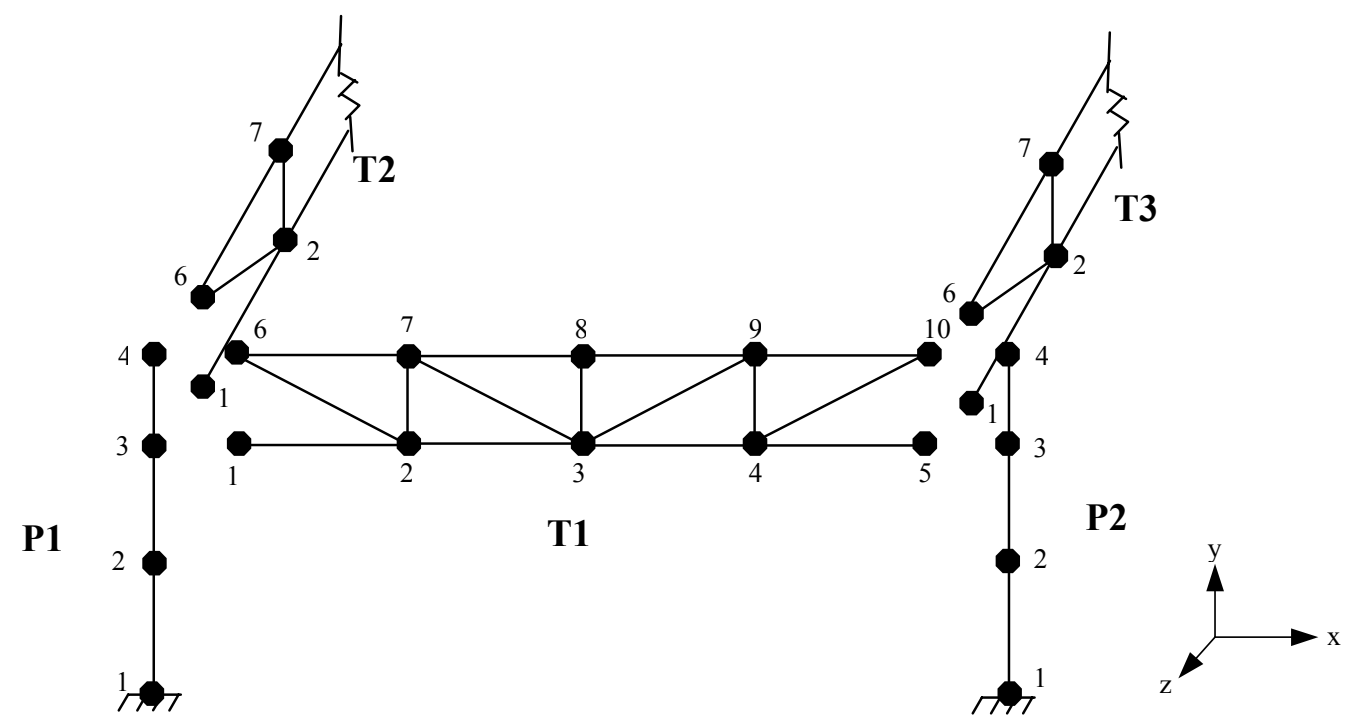

FIGURA 3.14 - Sistema estrutural dividido em subestruturas

e numeração dos nós individualmente.

Cada subestrutura conterá em seus dados:

- Nome da subestrutura.

- Tipo estrutural: pórtico ou treliça.

- Coordenadas nodais numeradas.

- Numeração das barras e suas incidências nodais.

- Características físicas e geométricas: E, G, $I_{x}, I_{y}, I_{z}, A$, ângulo $\alpha$.

- Carregamentos.

- Nós de apoio (restritos).

Os dados de cada subestrutura serão armazenados em arquivos próprios.

Até esta etapa, nada difere do lançamento de uma estrutura por um programa tradicional. Também, não há necessidade de indicar quais os nós que são de união entre as subestruturas. 
Posteriormente, todas as coordenadas nodais de todas as subestruturas são comparadas. Os nós com coordenadas iguais serão identificados como nós de união.

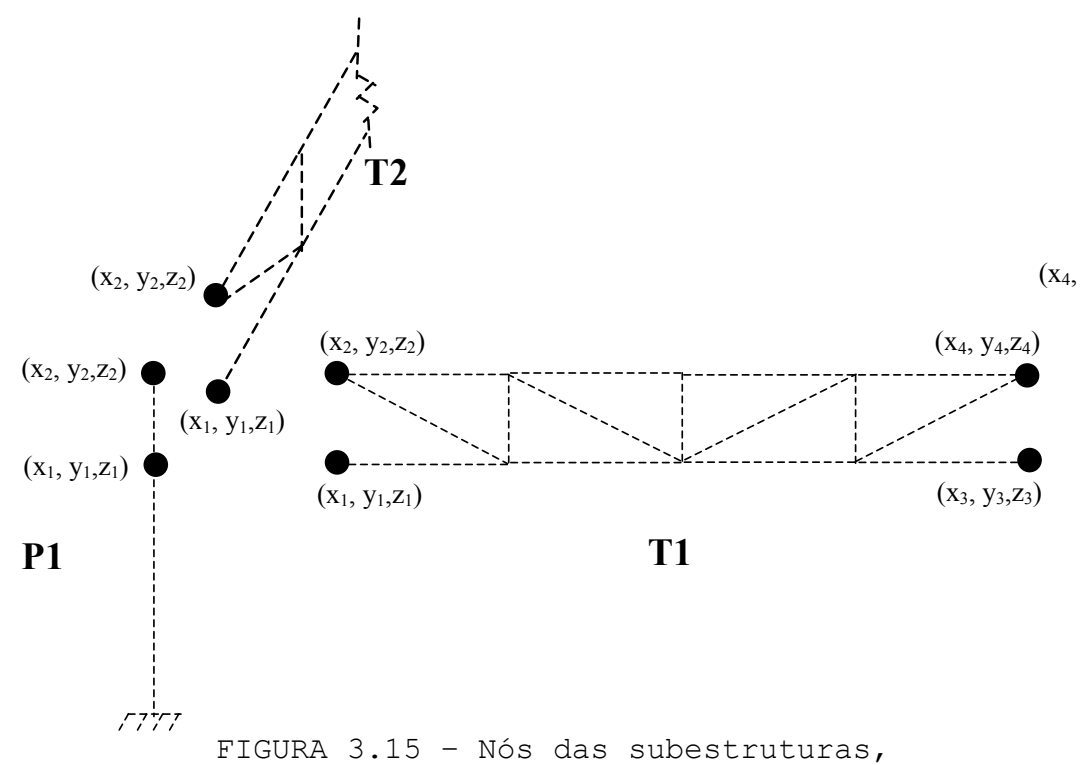

com coordenadas iguais

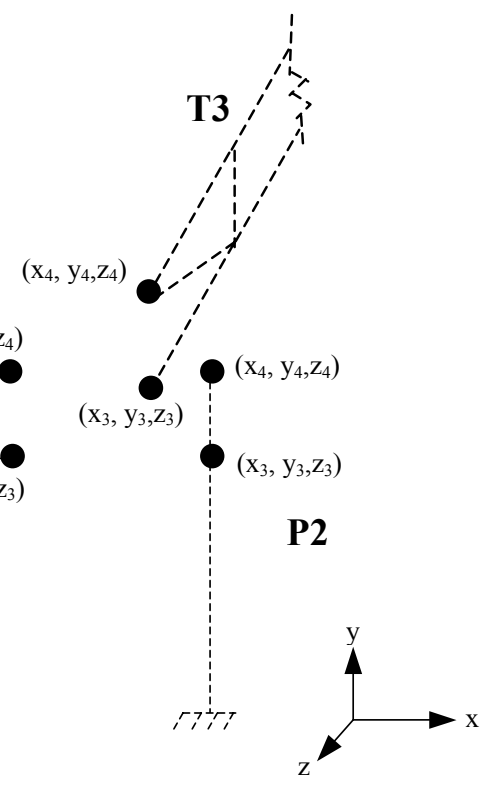

Deste processo resultam vetores e matrizes que serão constantemente consultados durante todo o programa

\section{4 - Renumeração das coordenadas}

Com a determinação dos nós de união, as coordenadas de cada subestrutura são renumeradas em função do grau de liberdade (apoio ou livre) e da condição de seu contorno (unidas ou não à outra subestrutura).

Para cada subestrutura são montados vetores auxiliares: Nrt, Srt, Nrb, Crb, onde:

N: $\quad$ No total de coordenadas internas livres.

Ncrb: $\quad \mathrm{N}^{\circ}$ total de coordenadas externas livres. 
Nrt: $\quad$ Matriz indicativo de coordenada

$$
\text { restringida ou não: }
$$

nrt $=1 \Rightarrow$ restringida.

nrt $=0 \Rightarrow$ livre para se deslocar.

Srt: Matriz indicativa da quantidade de coordenadas restringidas.

Nrb: Semelhante à Nrt, mas indicando as coordenadas de união às outras subestruturas:

$$
\begin{aligned}
& \mathrm{n} r \mathrm{~b}=1 \Rightarrow \text { coordenada externa. } \\
& \mathrm{n} r \mathrm{~b}=0 \Rightarrow \text { coordenada interna. }
\end{aligned}
$$

Crb: Semelhante a Srt, mas indicando a somatória de coordenadas externas.

jj[1..6]: Contém a numeração das coordenadas de cada nó.

- processo consiste em iniciar a renumeração a partir das coordenadas internas, dando sequência às coordenadas externas, e por último às coordenadas restringidas, conforme algoritmo abaixo.

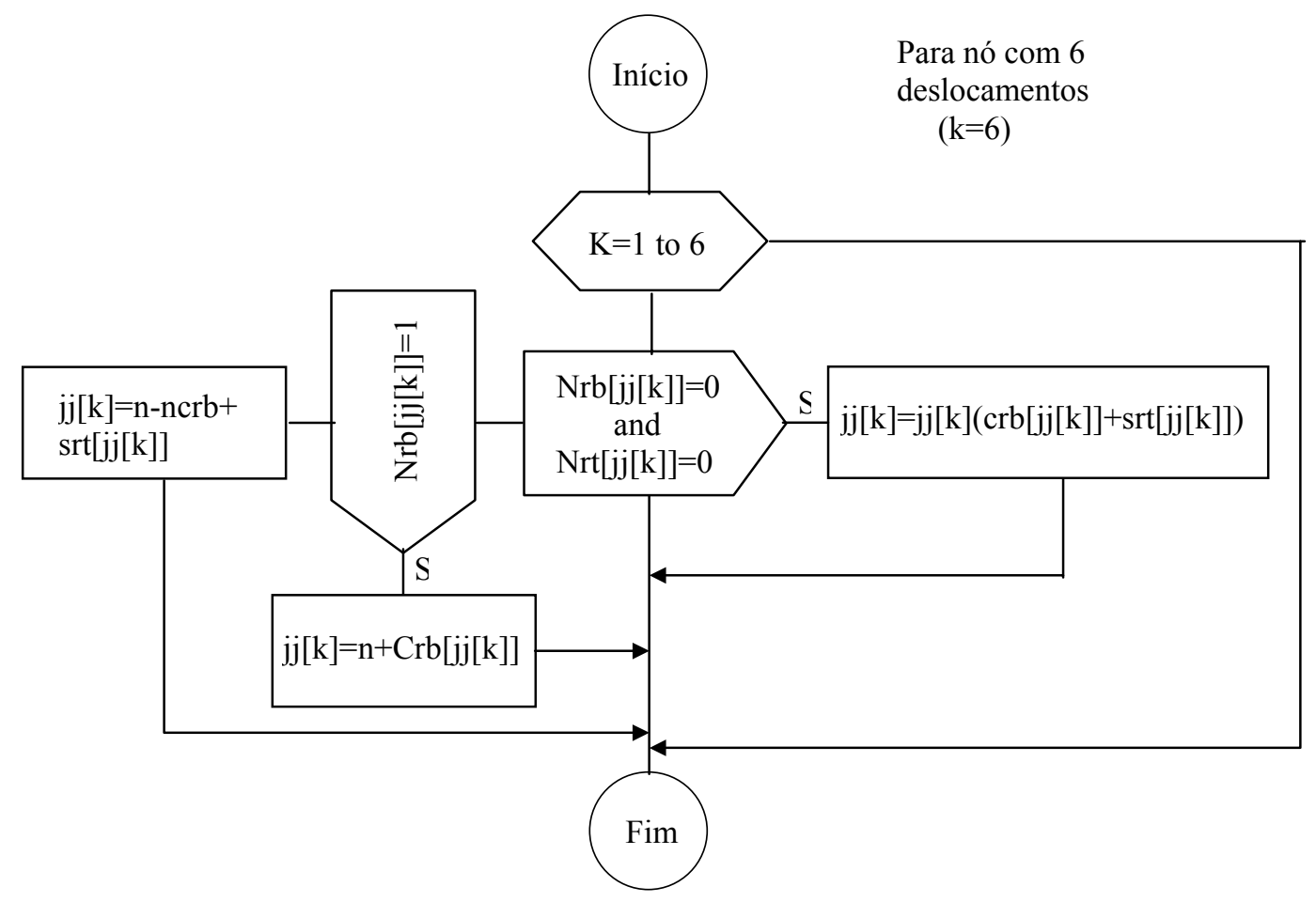


A subestrutura P1, como pórtico espacial, exemplifica o processo:
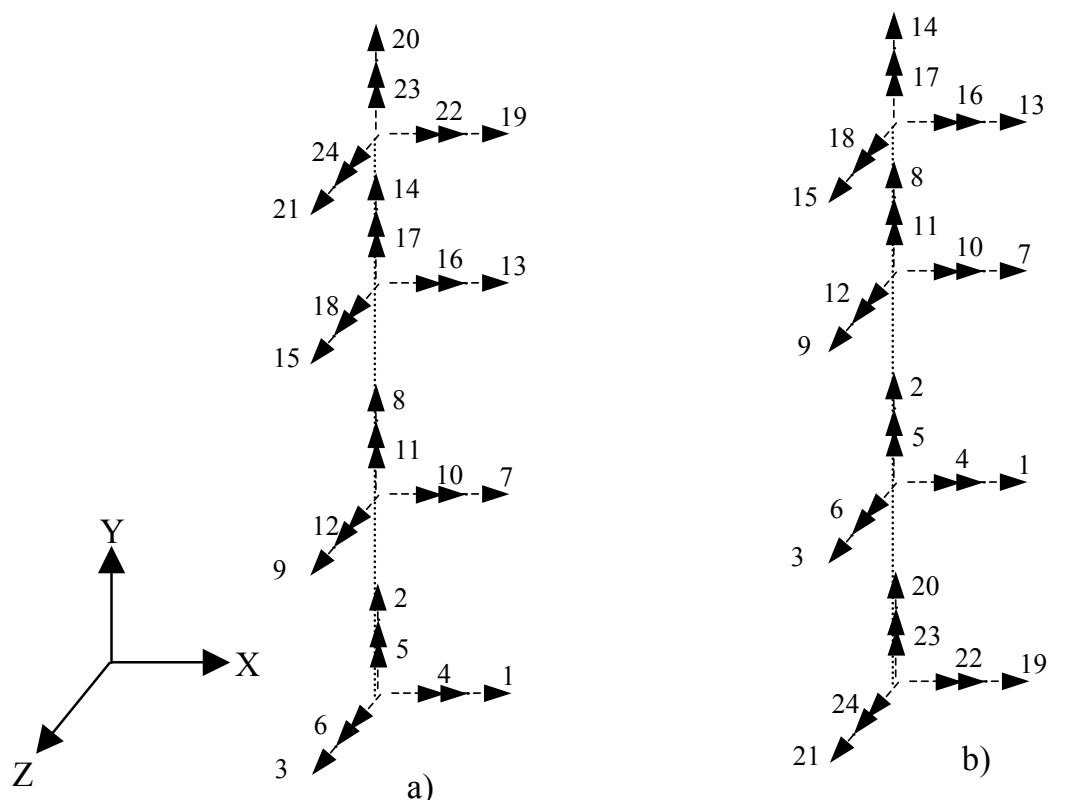

FIGURA 3.16 - Renumeração das coordenadas do pilar P1, da original (a) para a nova (b) .

\begin{tabular}{|l|c|c|c|c|c|c|c|c|c|c|c|c|c|c|c|c|c|c|c|c|c|c|c|c|}
\hline Coord & 1 & 2 & 3 & 4 & 5 & 6 & 7 & 8 & 9 & 10 & 11 & 12 & 13 & 14 & 15 & 16 & 17 & 18 & 19 & 20 & 21 & 22 & 23 & 24 \\
\hline Nrt & 1 & 1 & 1 & 1 & 1 & 1 & 0 & 0 & 0 & 0 & 0 & 0 & 0 & 0 & 0 & 0 & 0 & 0 & 0 & 0 & 0 & 0 & 0 & 0 \\
\hline Srt & 1 & 2 & 3 & 4 & 5 & 6 & 6 & 6 & 6 & 6 & 6 & 6 & 6 & 6 & 6 & 6 & 6 & 6 & 6 & 6 & 6 & 6 & 6 & 6 \\
\hline Nrb & 0 & 0 & 0 & 0 & 0 & 0 & 0 & 0 & 0 & 0 & 0 & 0 & 1 & 1 & 1 & 1 & 1 & 1 & 1 & 1 & 1 & 1 & 1 & 1 \\
\hline Crb & 0 & 0 & 0 & 0 & 0 & 0 & 0 & 0 & 0 & 0 & 0 & 0 & 1 & 2 & 3 & 4 & 5 & 6 & 7 & 8 & 9 & 10 & 11 & 12 \\
\hline
\end{tabular}

TABELA 3.1 - Valores dos vetores auxiliares para a subestrutura P1.

Com esta renumeração, cria-se condições para inserir corretamente os dados nas matrizes de rigidez correspondentes. 


\section{5 - Matrizes condensadas}

Com a etapa anterior definida, as matrizes de rigidez, $\mathbf{S}_{\mathbf{I I}}, \mathbf{S}_{\mathbf{I E}}, \mathbf{S}_{\mathbf{E E}}$, e os vetores de forças, $\mathbf{F}_{\mathbf{I}}$ e $\mathbf{F}_{\mathbf{E}}$, da subestrutura são montados.

Inicia-se, então, a primeira etapa do processo de condensação estática.

$$
\left[\begin{array}{cc}
\mathrm{S}_{\mathrm{II}} & \mathrm{S}_{\mathrm{IE}} \\
\mathrm{S}_{\mathrm{EI}} & \mathrm{S}_{\mathrm{EE}}
\end{array}\right]\left[\begin{array}{c}
\mathrm{U}_{\mathrm{I}} \\
\mathrm{U}_{\mathrm{E}}
\end{array}\right]=\left[\begin{array}{c}
\mathrm{F}_{\mathrm{I}} \\
\mathrm{F}_{\mathrm{E}}
\end{array}\right] \underset{\mathrm{ETAPA} 1}{\Rightarrow}\left[\begin{array}{cc}
\mathrm{S}_{\mathrm{II}}^{*} & \mathrm{~S}_{\mathrm{IE}}^{*} \\
0 & \mathrm{~S}_{\mathrm{EE}}^{*}
\end{array}\right]\left[\begin{array}{c}
\mathrm{U}_{\mathrm{I}} \\
\mathrm{U}_{\mathrm{E}}
\end{array}\right]=\left[\begin{array}{c}
\mathrm{F}_{\mathrm{I}}^{*} \\
\mathrm{~F}_{\mathrm{E}}^{*}
\end{array}\right]
$$

Onde são aplicadas as formulações 2.43 a 2.47 .

Estas matrizes são armazenadas, visando etapas posteriores.

Cabe ressaltar que todo o procedimento, descrito até aqui, é executado em uma única etapa para cada subestrutura, utilizando subrotinas adequadas ao tipo estrutural a que pertencem.

\section{6 - Solução dos deslocamentos externos}

A Segunda etapa do método das subestruturas consiste na solução do sistema de equações $\mathbf{S}_{\mathbf{C}} \mathbf{U}_{\mathbf{C}}=\mathbf{F}_{\mathbf{C}}$, formado pelas matrizes envolvendo os nós de união. 

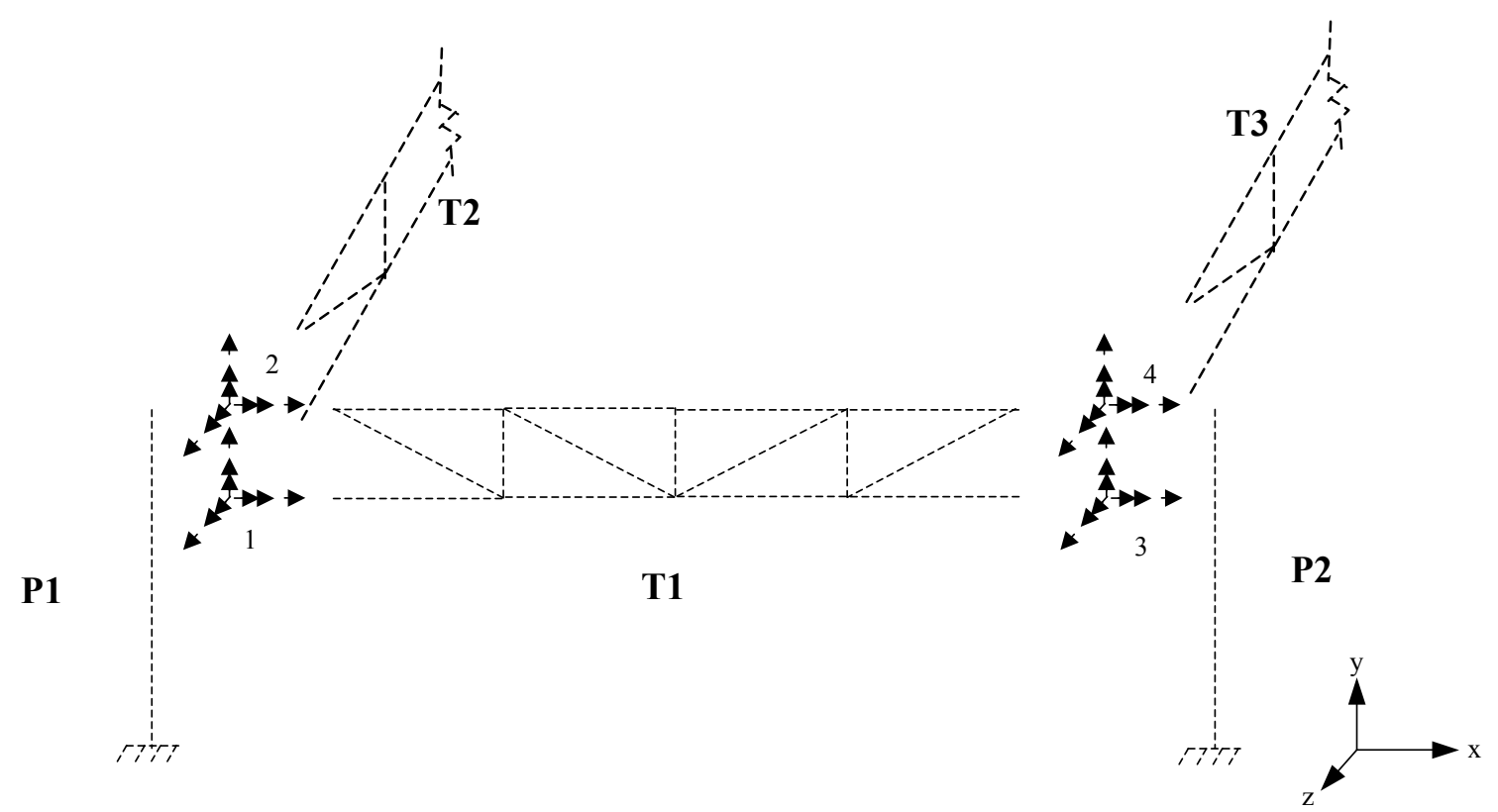

Figura 3.17 - Nós de união numerados para formação da $2^{\text {a }}$ etapa do processo.

A formação destas matrizes requer $\circ$ acoplamento correto das rigidezes e forças das subestruturas, contidas em $\mathrm{S}_{\mathrm{EE}}^{*}$ e $\mathrm{F}_{\mathrm{E}}^{*}$, considerando na somatória os elementos que correspondam aos mesmos deslocamentos e suas interações.

As matrizes $\mathbf{S}_{\mathbf{C}}, \quad \mathbf{F}_{\mathbf{C}}$ e $\mathbf{U}_{\mathbf{C}}$ são idealizadas para trabalharem com 6 deslocamentos por nó.

Exemplificando somente os dois primeiros nós de união do sistema: 

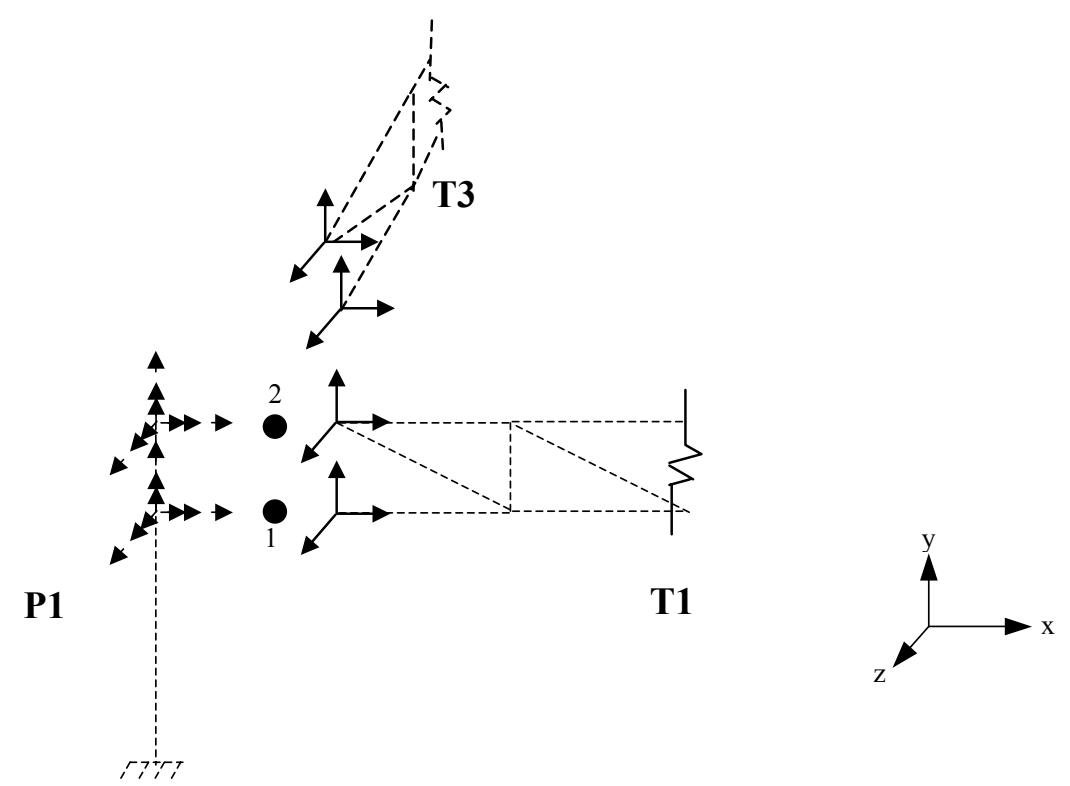

FIGURA 3.18 - Coordenadas das subestruturas a serem acopladas ao sistema de equações.

As rigidezes e forças, devidos aos nós de pórtico, serão acopladas ao sistema de equações em correspondência com a numeração adotada, considerando 6 deslocamentos por nó. As treliças serão acopladas em correspondência a 3 deslocamentos nodais.

Nesta fase, o programa realiza uma interação entre todas as subestruturas, no qual os dados são lidos dos arquivos previamente gravados, analisados e selecionados para serem inseridos no sistema de equações.

$\mathrm{Na}$ equação abaixo, as marcações com ' $x$ ' nas matrizes indicam as posições em que serão somadas as contribuições da treliça, considerando os 2 nós iniciais. 


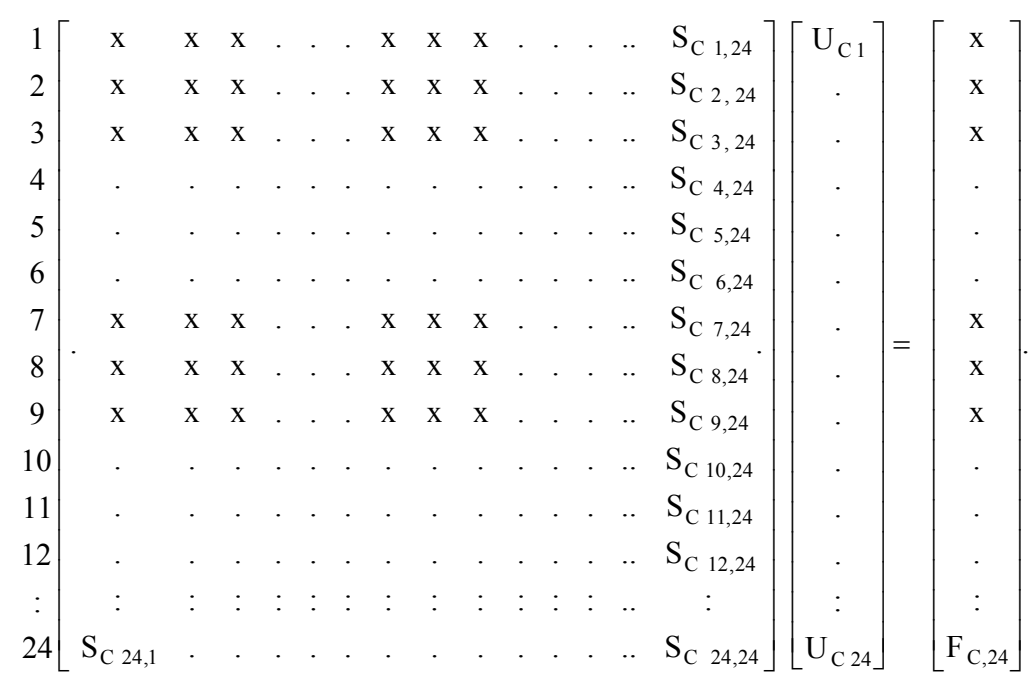

Da solução deste sistema linear, surgem os deslocamentos dos nós de união.

Salienta-se a importância desta fase em possibilitar a união de tipos estruturais diferentes. A introdução correta das grandezas e de suas interações, de cada subestrutura, no sistema de equações irá permitir a compatibilização correta dos deslocamentos nodais.

O fato de cada subestrutura ser analisada com suas subrotinas próprias, permite ampliar as possibilidades de se trabalhar com vários tipos estruturais diferentes, desde que esta fase seja adequadamente conduzida.

\section{7 - Deslocamentos Internos}

Correspondente à Etapa 3 do método das subestruturas. O programa volta a trabalhar com as subrotinas desenvolvidas para cada tipo estrutural.

Para cada subestrutura, os deslocamentos externos, obtidos de $\mathbf{U}_{\mathbf{C}}$ são inseridos adequadamente às matrizes $\mathbf{U}_{\mathbf{E}}$, e calculados os deslocamentos referentes às coordenadas 
internas, utilizando as formulações de 2.49 a 2.50, para a solução do sistema:

$$
\left[\begin{array}{cc}
\mathrm{S}_{\mathrm{II}}^{*} & \mathrm{~S}_{\mathrm{IE}}^{*} \\
0 & \mathrm{I}
\end{array}\right]\left[\begin{array}{c}
\mathrm{U}_{\mathrm{I}} \\
\mathrm{U}_{\mathrm{E}}
\end{array}\right]=\left[\begin{array}{c}
\mathrm{F}_{\mathrm{I}}^{*} \\
\mathrm{U}_{\mathrm{E}}
\end{array}\right] \underset{\text { ETAPA 3 }}{\Rightarrow}\left[\begin{array}{c}
\mathrm{U}_{\mathrm{I}} \\
\mathrm{U}_{\mathrm{E}}
\end{array}\right]
$$

Com todos os deslocamentos obtidos, finaliza-se a análise com a determinação das ações de extremidade das barras e as reações de apoios, inerentes ao método dos deslocamentos. O programa emite um relatório final, para cada subestrutura, contendo todos os dados introduzidos, juntamente com os resultados da análise. 


\section{8 - Técnicas computacionais.}

Dentre algumas técnicas computacionais para otimização do processamento, optou-se pelo armazenamento em linha das matrizes com características simétricas, possibilitando armazenar apenas a diagonal principal e a parte triangular superior delas.

Foram desenvolvidas subrotinas adequadas para a formação das matrizes de rigidez em forma particionada, levando em consideração as características de cada tipo de subestrutura.

As matrizes simétricas $\mathrm{S}_{\mathrm{II}}, \mathrm{S}_{\mathrm{EE}} \quad \mathrm{e} \quad \mathrm{S}_{\mathrm{C}}$ serão armazenadas num vetor em linha, e a matriz retangular $\mathrm{S}_{\mathrm{IE}}$ em arranjo normal, ou seja, retangular.

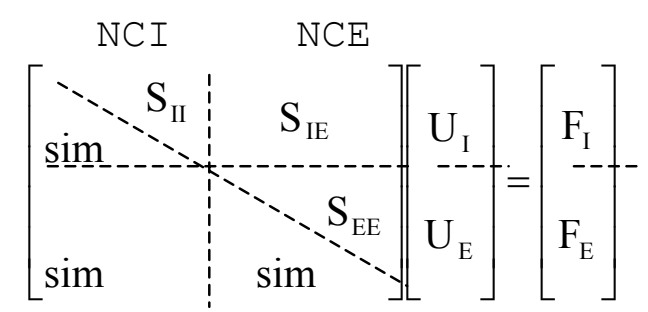

Matriz de rigidez particionada

$\mathrm{NCI}=\mathrm{N}^{\circ}$ de coordenadas internas

$\mathrm{NCE}=\mathrm{N}$ - de coordenadas externas

modelo:

O arranjo das matrizes simétricas em linha seguirá o 


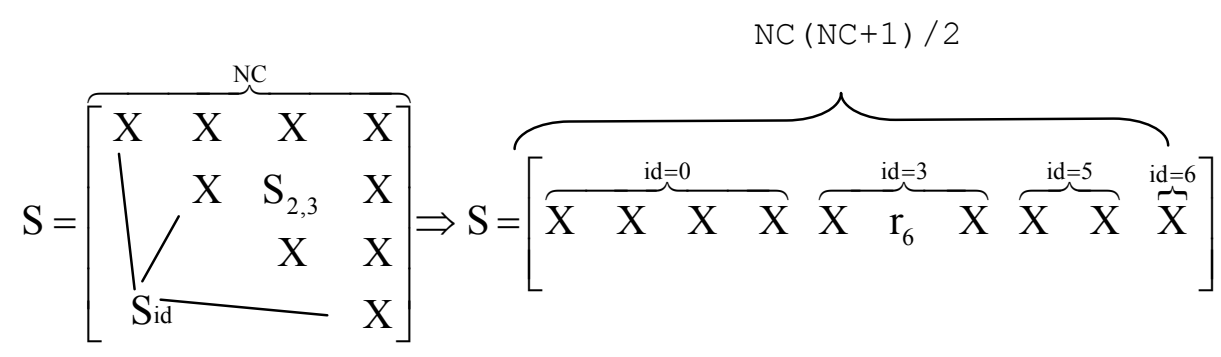

$$
\begin{aligned}
& i d=(i-1)(2 \mathrm{NC}-i) / 2
\end{aligned}
$$

Posição no vetor será dado por $S_{i d+j}$

Para as matrizes particionadas, ficam as dimensões:

$$
\begin{aligned}
& \mathrm{S}_{\mathrm{II}}=(\mathrm{NCI}(\mathrm{NCI}+1) / 2) \\
& \mathrm{S}_{\mathrm{EE}}=(\mathrm{NCE}(\mathrm{NCE}+1) / 2) \\
& \mathrm{S}_{\mathrm{IE}}=(\mathrm{NCI}, \mathrm{NCE})
\end{aligned}
$$

As subrotinas desenvolvidas para processarem tipos diferentes de elementos estruturais estão, na realidade, inseridas em apenas um código computacional.

As grandezas que envolvem pórtico tridimensional são relacionadas por índices que variam de 1..6, para treliça espacial variam de $1 . .3$.

A seqüência das grandezas seguem a ordenação:

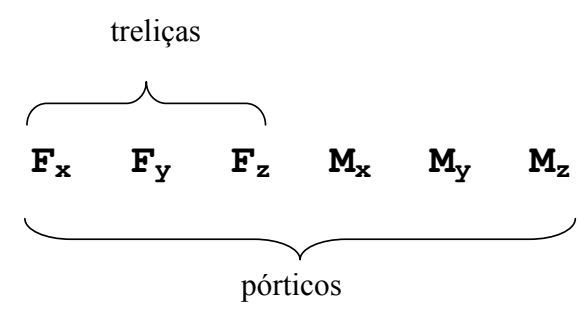

Devido a esta ordenação, o acesso aos dados dentro de matrizes ou vetores se dá com respeito aos índices definidos, diminuindo de sobremaneira a quantidade de códigos computacionais necessários. 


\section{9 - Subrotinas Principais e Fluxograma.}

A subrotinas principais que compõe o programa são descritas aqui, de uma forma sucinta. São elas:

- ORGAN-1: Este programa detecta as coordenadas comuns (de contorno) entre as subestruturas, comparando as coordenadas cartesianas de cada nó e também verificando as restrições dos nós. Somente coordenadas com grau de liberdade serão consideradas.

- MONTA_RIG: Na formação das matrizes $\mathrm{S}_{\mathrm{II}}, \mathrm{S}_{\mathrm{EE}}$ ou $\mathrm{S}_{\mathrm{IE}}$, de cada subestrutura, a matriz de rigidez de cada barra é verificada e seus elementos inseridos nestas matrizes, conforme a ocorrência dos graus de liberdade e de contorno. O vetor de forças nodais de cada barra também é inserido no vetor $F_{I}$ ou $F_{E}$, correspondentemente.

- DECOMP-1: Realiza o processo de decomposição para obter as matrizes condensadas.

$$
\left[\begin{array}{cc}
\mathrm{S}_{\mathrm{II}} & \mathrm{S}_{\mathrm{IE}} \\
\operatorname{sim} & \mathrm{S}_{\mathrm{EE}}
\end{array}\right]\left[\begin{array}{c}
\mathrm{U}_{\mathrm{I}} \\
\mathrm{U}_{\mathrm{E}}
\end{array}\right]=\left[\begin{array}{c}
\mathrm{F}_{\mathrm{I}} \\
\mathrm{F}_{\mathrm{E}}
\end{array}\right] \underset{\text { DECOMP-1 }}{\Rightarrow}\left[\begin{array}{cc}
\mathrm{S}_{\mathrm{II}}^{*} & \mathrm{~S}_{\mathrm{IE}}^{*} \\
& \mathrm{~S}_{\mathrm{EE}}^{*}
\end{array}\right]\left[\begin{array}{c}
\mathrm{U}_{\mathrm{I}} \\
\mathrm{U}_{\mathrm{E}}
\end{array}\right]=\left[\begin{array}{c}
\mathrm{F}_{\mathrm{I}}^{*} \\
\mathrm{~F}_{\mathrm{E}}^{*}
\end{array}\right]
$$

- ORGAN-2: Forma as matrizes $\mathrm{S}_{\mathrm{C}}$ e $\mathrm{F}_{\mathrm{C}}$, que são montadas a partir das matrizes $\mathrm{S}_{\mathrm{EE}}{ }^{*}$ e $\mathrm{F}_{\mathrm{E}}^{*}$ de cada subestrutura. A matriz $\mathrm{S}_{\mathrm{C}}$ trabalha com 6 deslocamentos 
por nó. Esta sub-rotina acopla adequadamente as matrizes de uma treliça, que trabalham com 3 deslocamentos por nó.

- Solsis: Resolve o sistema final de equações da estrutura condensada, composta por $\mathrm{S}_{\mathrm{C}}$ e $\mathrm{F}_{\mathrm{C}}$, obtendo os deslocamentos das coordenadas comuns, e que são armazenados em $\mathrm{U}_{\mathrm{C}}$.

$$
\overbrace{\left[\mathrm{S}_{\mathrm{C}}\right]}^{\text {NCRV }}\left[\mathrm{U}_{\mathrm{C}}\right]=\left[\mathrm{F}_{\mathrm{C}}\right] \underset{\text { SOLSIS }}{\Rightarrow}\left[\mathrm{U}_{\mathrm{C}}\right]
$$

- DECOMP-2: Uma subrotina interna, "FCFE", espalha os deslocamentos externos, obtidos de "SOLSIS", de volta às respectivas subestruturas. Depois, por um processo de retrosubstituição, os deslocamentos das coordenadas internas $\mathrm{U}_{\mathrm{I}}$, para cada subestrutura, são determinados.

$$
\left[\begin{array}{cc}
\overbrace{\mathrm{S}_{\mathrm{II}}^{*}}^{\mathrm{N}} & \overbrace{\mathrm{SEE}}^{\mathrm{NCRB}} \\
& \mathrm{I}
\end{array}\right]\left[\begin{array}{c}
\mathrm{U}_{\mathrm{I}} \\
\mathrm{U}_{\mathrm{E}}
\end{array}\right]=\left[\begin{array}{c}
\mathrm{F}_{\mathrm{I}}^{*} \\
\mathrm{U}_{\mathrm{E}}
\end{array}\right] \underset{\text { DECOMP-2 }}{\Rightarrow}\left[\begin{array}{c}
\mathrm{U}_{\mathrm{I}} \\
\mathrm{U}_{\mathrm{E}}
\end{array}\right]
$$


o fluxograma relativo

à

seqüência adotada

no programa é:

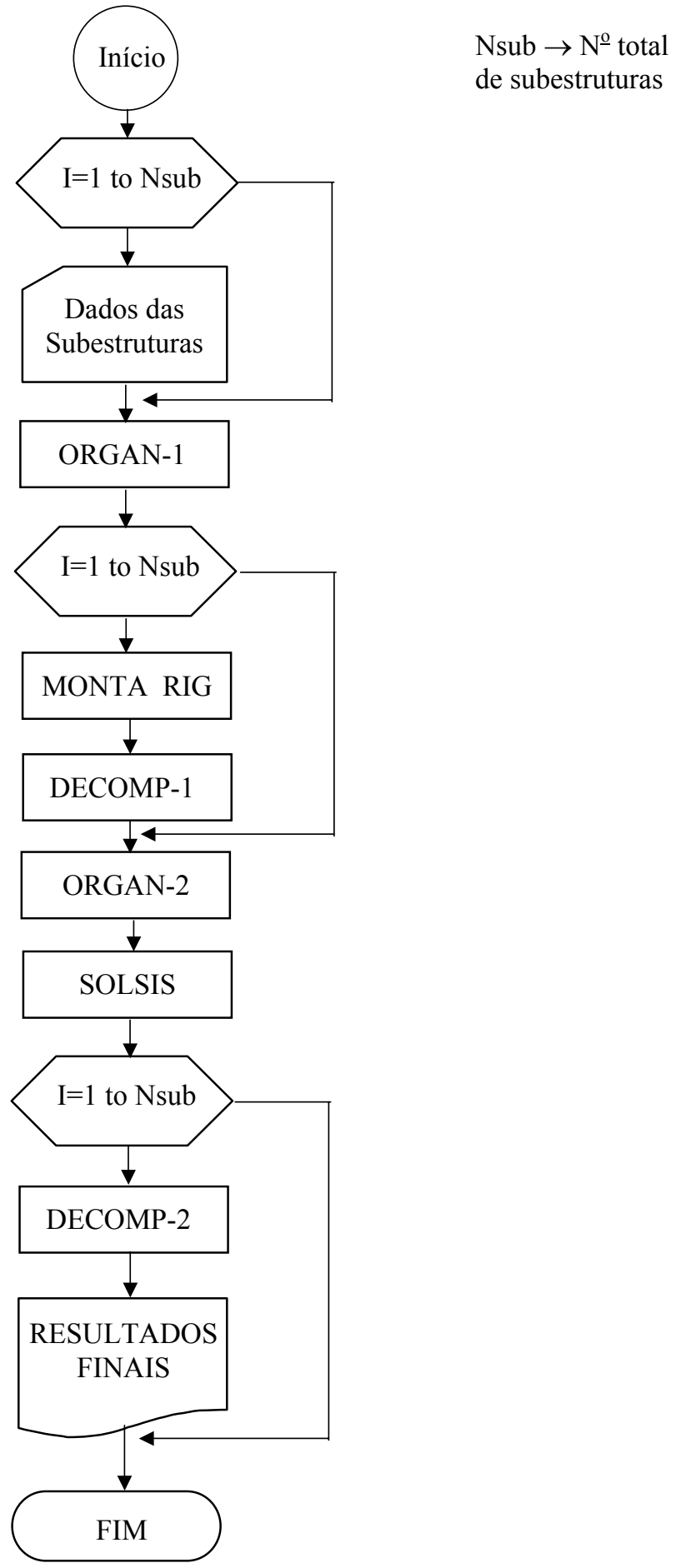

FIGURA 3.19 - Fluxograma 


\subsection{0 - Interface gráfica para aquisição de dados}

Para automatizar os procedimentos de cálculo descritos nos capítulos anteriores, visando a análise de estruturas particionadas, foram desenvolvidos programas que utilizam a linguagem PASCAL, para a análise estrutural, e AutoLisp para a interface gráfica.

Cada subestrutura será tratada individualmente, desde a introdução dos dados até ao final do processo, com os resultados da análise somente dela. Característica inerente ao método da subestruturação.

Foram desenvolvidas subrotinas em linguagem Autolisp, que serão utilizadas conjuntamente com o editor gráfico AutoCad. Desta forma, torna-se disponível uma extensa biblioteca de comandos gráficos já implementados.

Tem como maior vantagem a visualização imediata durante a discretização estrutural, permitindo correções ou modificações em tempo real.

Neste ambiente a estrutura inteira é desenhada em forma de linhas, como se fosse um desenho comum. As funções (comandos) desenvolvidas em Autolisp, e representadas por ícones, conforme FIGURA 3.20, irão acrescentar informações neste desenho, transformando-o em um objeto representativo de uma estrutura. 


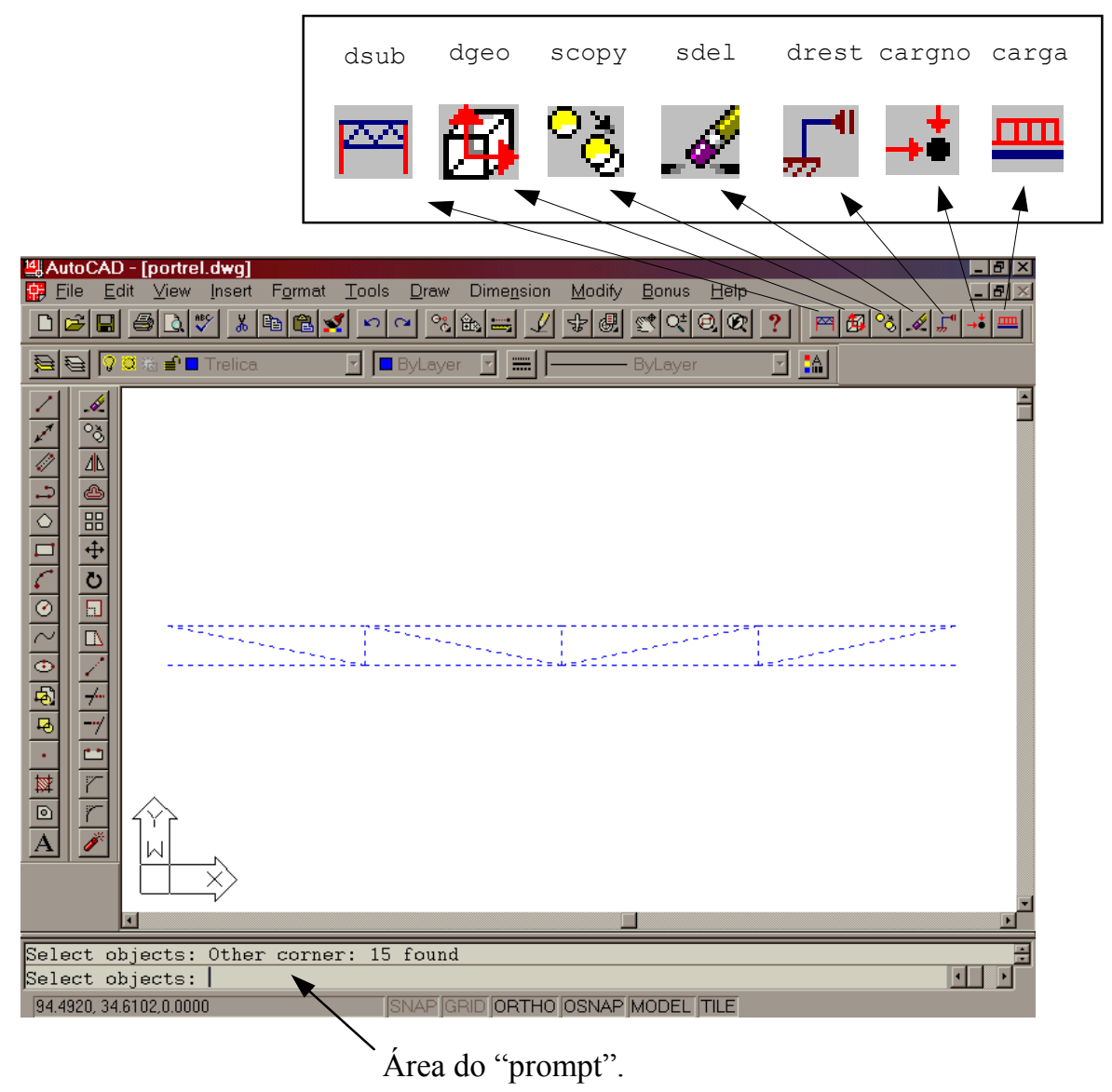

FIGURA 3.20 - Ícones(comandos), em destaque, acrescidos ao programa Autocad, e subestrutura
selecionada (tracejada).

\subsection{0 .1 - Discretização de uma subestrutura}

Uma subestrutura é desenhada na tela em forma de linhas, e em suas dimensões reais, conforme FIGURA 3.20.

- Autocad considera uma linha como sendo uma entidade, criando internamente um banco de dados contendo todos os atributos dela, como: cor, layer, coordenadas iniciais, etc... 
Ao mesmo tempo, também cria um "nome" para esta entidade. Este "nome", em termos computacionais, é um "handle" (semelhante a um endereço hexadecimal) que direciona para o banco de dados da entidade. Através deste "nome" é que se pode acessar os dados desta entidade.

As característica da entidade, contidas neste banco de dados, são acessadas por índices, como os índices de um vetor. Por exemplo, os índices 10 e 11 fornecem, respectivamente, as coordenadas do nó inicial e do nó final da linha desenhada, que são os principais interesses para este programa. Este banco de dados é pesquisado por funções específicas dentro do Autolisp.

É oportuno observar, ainda, que o Autocad sempre atualiza as informações contidas neste banco de dados. Por exemplo, quando uma linha é movida para outra posição, os dados referenciados pelos índices 10 e 11 são atualizados automaticamente.

Outro procedimento realizado pelo Autocad, e explorado intensamente pelo programa, é o de selecionar entidades, de modo que funções sejam aplicadas somente nelas.

Neste processo de seleção, os "nomes" das entidades selecionadas são armazenadas numa lista (vetor), podendo ser acessadas por comandos em AutoLisp.

A formação de uma subestrutura será realizada por uma função, desenvolvida em Autolisp, chamada de "dsub". Inicialmente, são selecionadas linhas (barras), desenhadas anteriormente, e que irão compor a subestrutura, conforme FIGURA 3.20. Entidades selecionadas sempre se mostram com linhas tracejadas. 
Os nomes de todas as linhas serão armazenadas em uma lista. Listas, em AutoLisp, são estruturas computacionais semelhantes a vetores ou matrizes.

Posteriormente, a função solicita os valores do módulo de elasticidade transversal e longitudinal (G e E) e o nome da subestrutura, para referências futuras.

Estes dados são solicitados e digitados na região de "prompt" da tela.

Desta maneira, fica definida uma subestrutura, com seus dados dispostos em uma lista, da seguinte forma:

$$
(<\text { "nome das barras" }>\text {, nomesub , } \mathrm{E}, \mathrm{G})
$$

Conforme novas subestruturas vão sendo definidas, novas listas são montadas.

Da mesma forma, estas listas são armazenadas em outra lista, que conterá todas as subestruturas. Semelhante a um vetor, onde cada elemento deste vetor é uma lista (subestrutura). Toma a seguinte forma:

( (<nomebarras>, nomesub1, E, G) (<nomebarras $>$, nomesub2, E, G) ....)

Cada subestrutura é referenciada por um índice dentro desta lista.

\subsection{2 - Características das barras}

A função "dgeo", desenvolvida em Autolisp, irá acrescentar às barras as suas propriedades, que são: Área 
da seção transversal, momentos de Inércia $\left(I_{x}, I_{y}\right.$ e $\left.I_{z}\right)$, e ângulo $\alpha$.

As barras com mesmas características são selecionadas através de cliques com o mouse (processo de seleção do AutoCad). Cada barra, então, é identificada pelo seu "nome" (handle). Forma-se uma lista para cada barra, contendo:

$$
\left(<\text { nomebarra }>, A, I_{x}, I_{y}, I_{z}, \alpha\right)
$$

Da mesma forma anterior, cria-se uma lista contendo todas as listas das barras:

\footnotetext{
( (<nomebarra1 $\left.>, A, \quad I_{x}, I_{y}, I_{z}, \alpha\right)$ (<nomebarra2>, A, $\left.I_{x}, I_{y}, I_{z}, \alpha\right) \ldots$ )

Todas as barras, de todas as subestruturas, e suas características, estarão contidas nesta lista.

Os dados são solicitados e introduzidos na região de "prompt" da tela.
}

\subsection{3 - Cargas aplicadas nos nós.}

Outra função desenvolvida em Autolisp, denominada "cargno", conforme ícone na FIGURA 3.20, de forma semelhante às outras funções, irá obter informações da geometria do desenho para serem acrescidas aos dados referentes às subestruturas.

Inicialmente, a função solicita o valor das forças $\left(F_{x}, F_{y}, F_{z}\right)$ a serem aplicadas. Estes valores formam uma 
lista. Em seguida, solicita os momentos $\left(M_{x}, M_{y}, M_{z}\right)$, formando outra lista.

Os nós, a receberem estes carregamentos, são selecionados por cliques do mouse, conforme FIGURA 3.21 .

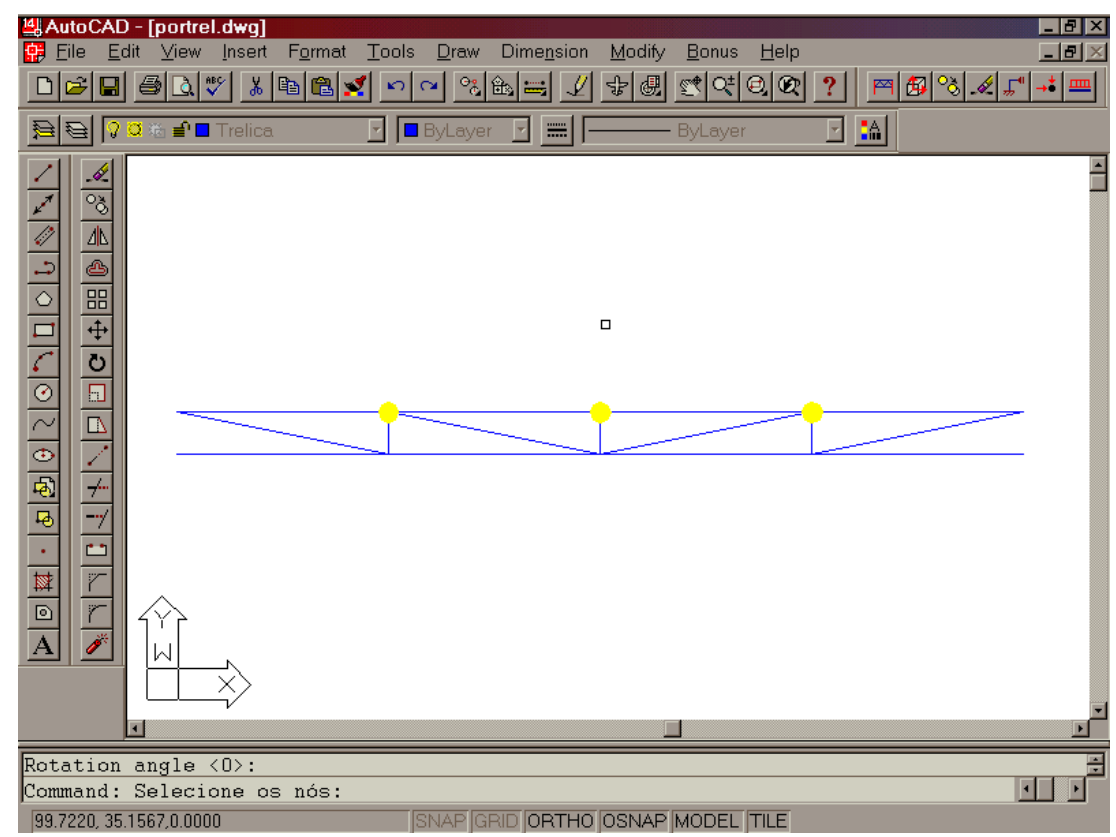

$\begin{aligned} \text { FIGURA 3.21 - } & \text { Nós selecionados em amarelo } \\ & \text { para receberem carregamentos. }\end{aligned}$

Como mencionado anteriormente, é importante que os dados sejam relacionados ao "nome" (handle) da entidade. - programa consulta o banco de dados do Autocad, especificamente os índices 10 e 11 de cada linha, verificando a igualdade entre as coordenadas dos pontos clicados e as coordenadas iniciais das entidades. Detectada a igualdade, o nó selecionado para o carregamento fica identificado pelo "nome" da barra e pelo seu índice (10 ou 11).

Para cada barra será criada uma lista, na forma:

$\left(<\right.$ nomebarra $>$, índice, $\left.\left(\mathrm{F}_{\mathrm{x}}, \mathrm{F}_{\mathrm{y}}, \mathrm{F}_{\mathrm{z}}\right),\left(\mathrm{M}_{\mathrm{x}}, \mathrm{M}_{\mathrm{y}}, \mathrm{M}_{\mathrm{z}}\right)\right)$ 
Uma lista contendo informações de todas as barras, será:

( (<nomebar1>, código, $\left.\left(\mathrm{F}_{\mathrm{x}}, \mathrm{F}_{\mathrm{Y}}, \mathrm{F}_{\mathrm{z}}\right), \quad\left(\mathrm{M}_{\mathrm{X}}, \mathrm{M}_{\mathrm{Y}}, \mathrm{M}_{\mathrm{z}}\right)\right)$ (<nomebar2>, código, $\left.\left.\left(\mathrm{F}_{\mathrm{x}}, \mathrm{F}_{\mathrm{y}}, \mathrm{F}_{\mathrm{z}}\right),\left(\mathrm{M}_{\mathrm{x}}, \mathrm{M}_{\mathrm{y}}, \mathrm{M}_{\mathrm{z}}\right)\right) \ldots\right)$

O código será o número 10 ou 11, indicando que a coordenada selecionada será o nó inicial ou final da entidade.

As forças são solicitadas e introduzidas na região de "prompt" da tela.

Ao longo de todo o programa, todos os dados introduzidos são associados às entidades através do seu "nome". Percebe-se, com isto, uma interação significativa entre os dados de uma subestrutura com a estrutura interna de dados do Autocad.

\subsection{4 - Cargas aplicadas nas barras}

A função "carga", de forma semelhante a anterior, irá formar a lista de carregamentos aplicados nas barras, também relacionadas ao "nome" da entidade.

- programa solicita o valor do carregamento, a distância, relativa à barra, no qual ele inicia e a distância final, por onde ele se estende, como visto no item 3.2.2, à página 41 .

As barras que irão receber o carregamento são selecionadas pelo mouse, conforme FIGURA 3.22. 


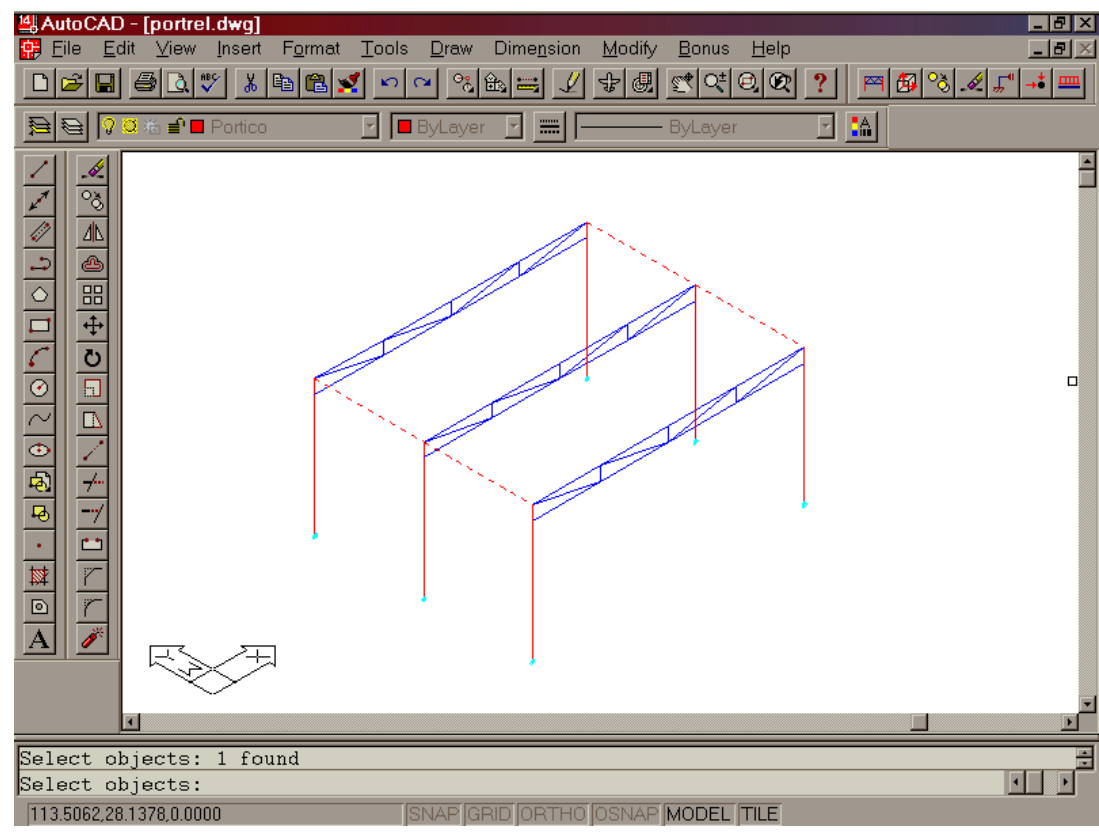

FIGURA 3.22- Barras selecionadas (tracejadas) para receberem carregamentos.

Para cada barra é montada uma lista, contendo:

(<nomebarra $>$, di, df, carga)

di: distância inicial.

df: distância final.

carga: valor do carregamento.

nomebarra: "nome" da entidade.

A lista contendo todas as barras carregadas será:

((<nomebarra1>, di, df, carga) (<nomebarra2>, di, df, carga)...) 


\subsection{0 .5 - Restrições de apoio}

A função "drest" monta uma lista contendo os nós que estarão com deslocamentos impedidos (vínculos de apoio).

- programa solicita os dados referentes aos deslocamentos correspondentes a $F_{x}, F_{y}, F_{z}$, depois os dados referentes à $M_{x}, M_{y}, M_{z}$.

Introduzindo o número 1 , para estes dados, indica restrição ao deslocamento, o número 0 indica grau de liberdade.

Os nós são selecionados pelo mouse, conforme FIGURA 3.23, onde também aparece uma nova subestrutura, tipo pórtico, que já foi discretizada.

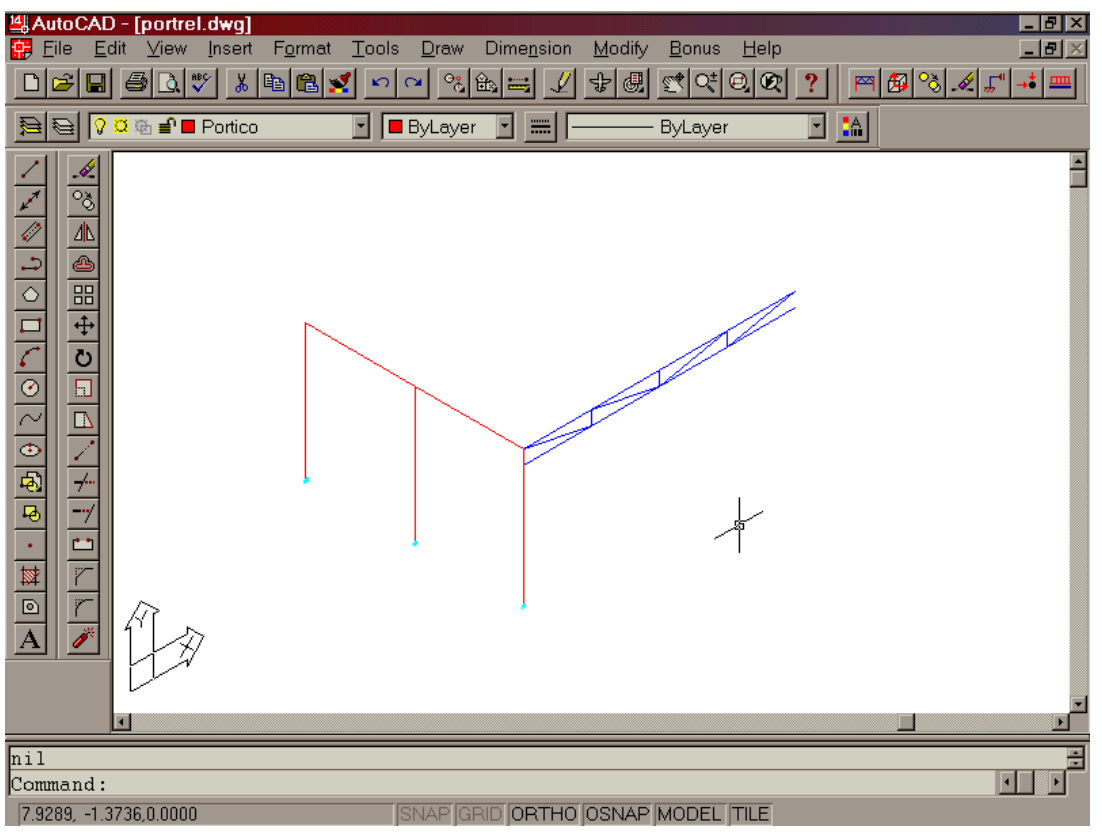

FIGURA 3.23 - Nós de apoio setados em azul claro 
A lista montada para cada nó é da forma:

("nomebarra", índice, $\mathrm{F}_{\mathrm{x}}, \mathrm{F}_{\mathrm{y}}, \mathrm{F}_{\mathrm{z}}, \mathrm{M}_{\mathrm{x}}, \mathrm{M}_{\mathrm{y}}, \mathrm{M}_{\mathrm{z}}$ )

A lista contém o "nome" da entidade, e o índice será - número 10 ou 11, indicando ser uma coordenada inicial ou final da entidade. De forma semelhante à lista criada pela função "cargno", vista anteriormente.

A lista contendo todos os nós restritos é:

( ("nomebar1", código, $F_{\mathrm{x}}, \mathrm{F}_{\mathrm{y}}, \mathrm{F}_{\mathrm{z}}, \mathrm{M}_{\mathrm{x}}, \mathrm{M}_{\mathrm{y}}, \mathrm{M}_{\mathrm{z}}$ ) ("nomebar2", código, $\left.F_{x}, F_{y}, F_{z}, M_{x}, M_{y}, M_{z}\right) \ldots$ )

\subsection{0 .6 - Outras funções}

As funções vistas até aqui são consideradas básicas para a completa discretização de uma subestrutura. Novas subestruturas acrescidas passam por todo este processo.

Visando automatizar melhor 0 lançamento das subestruturas, foi desenvolvida a função "scopy", que copia subestruturas inteiras para outras posições.

A subestrutura a ser copiada é selecionada por um simples clique do mouse em qualquer uma de suas barras. O nome desta barra é detectado. Em seguida é feita uma busca na lista de subestruturas para saber qual delas contém a barra.

A subestrutura detectada contém, em sua lista, o "nome" de todas as barras que a compõe. Através do "nome" é que o Autocad reconhece as entidades (linhas) e então seleciona-as para o processo de cópia. 


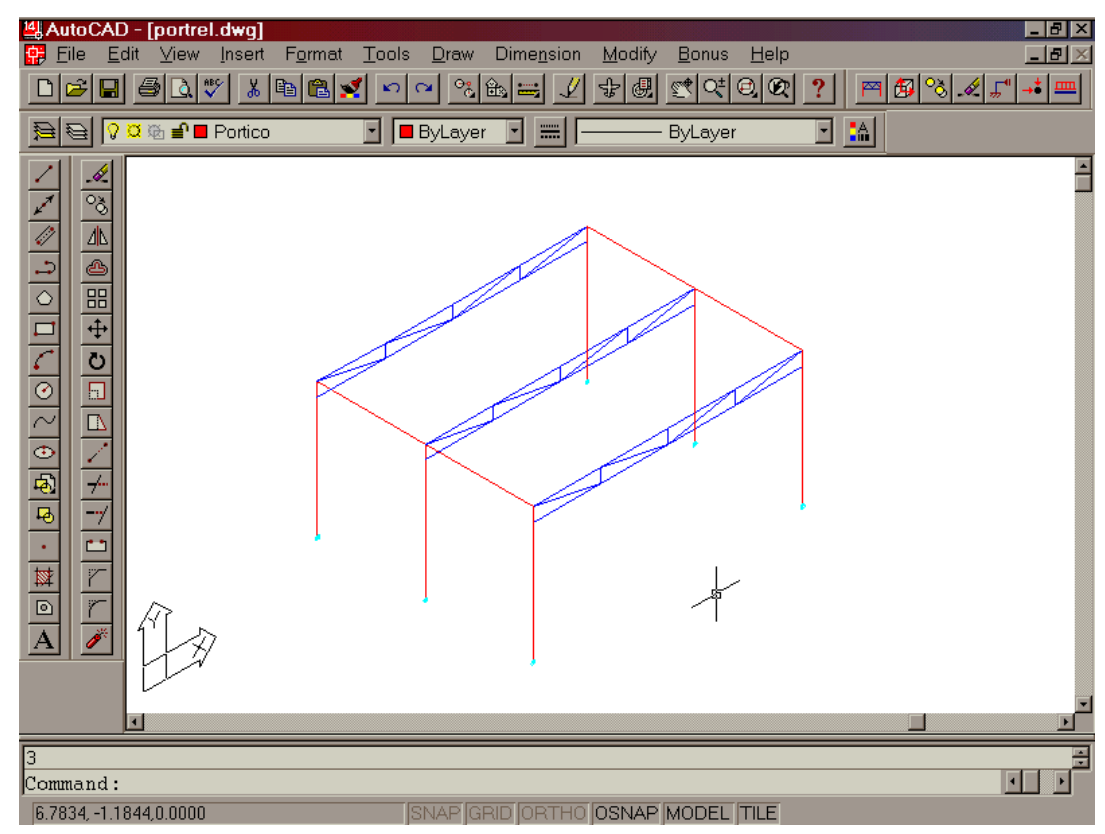

FIGURA 3.24 - Novas subestruturas que foram criadas pelo processo de "scopy".

Após a cópia, conforme FIGURA 3.24, o Autocad criou novas entidades (linhas). A função "scopy" as detecta e armazena seus "nomes" numa lista. Esta lista, então, é adicionada à lista de subestruturas.

Nesta nova subestrutura deverão ser aplicados todos os passos anteriores, como aplicação de cargas, restrição de nós, etc....

Caso a nova subestrutura tenha alguns aspectos semelhantes à que originou, a função "scopy" também poderá:

- copiar as características das seções transversais das barras, adicionando-as à lista criada em "dgeo", de forma automática.

- copiar os carregamentos nodais, acrescentando-os 
automaticamente à lista criada para cargas nodais.

- copiar os carregamentos das barras e acrescentalos à lista criada para cargas nas barras.

Desta forma, o processo de discretização da estrutura completa torna-se mais versátil.

A função "sdel" deleta uma subestrutura completa que já tenha sido criada. Ela é selecionada clicando-se com o mouse em uma de suas barras. Em seguida, a função interage com o Autocad para que o seu desenho seja eliminado da tela. Depois elimina todos os dados referentes aos "nomes" daquelas entidades nas listas criadas.

Foram desenvolvidas também outras funções que permitem correções de quaisquer dados introduzidos em quaisquer uma das fases, e que não serão descritas em seus pormenores.

\subsection{0 .7 - Arquivos de dados}

Quando todas as subestruturas se encontrarem discretizadas, o programa irá gravar os dados em em arquivos, de forma adequada, para posterior leitura e processamento pelo programa responsável pela análise estrutural.

Como já visto, cada subestrutura terá seus arquivos próprios, contendo todos os dados introduzidos referentes a ela e às suas barras. 
Será gravado também um arquivo que conterá a quantidade e os nomes das subestruturas, para que 0 programa de análise estrutural possa se orientar.

A distinção entre treliça e pórtico será feita pela cor do desenho. Nas figuras anteriores, percebe-se os pórticos desenhados em vermelho e as treliças em azul.

\subsection{1 - Editor em modo texto}

Apesar da interface gráfica predominar nos meios computacionais, os dados da estrutura quando introduzidos por meio de texto processado também condicionam a uma maneira confortável de trabalhar. Apenas não se visualiza em tempo real a estrutura discretizada.

Acompanhando o mesmo procedimento anterior, irá se editar uma estrutura por vez.

O editor elaborado para este programa tem sua tela dividida em duas partes. A da esquerda é para a introdução dos dados da estrutura. A da direita é reservada para a "Ajuda" (Help) durante a digitação dos dados.

O texto deverá conter palavras chaves seqüenciais para definir as características dos dados introduzidos. São elas:

- Gident: Define a sessão para introduzir a identificação da estrutura, como: nome, no de barras e nós, tipo estrutural, módulos de elasticidade longitudinal e transversal. 
- Gnos: Significando gerador de nós. São introduzidos os números dos nós e suas coordenadas. Possibilita gerar vários nós em um comando.

- Gtipo: Onde é definido, por um número, as características de uma barra, como: Momentos de Inércia, área e ângulo de giro $\alpha$ da seção transversal.

- Gbar: Onde as barras são caracterizadas por suas incidências (nós finais) e ao tipo que pertencem, definido anteriormente em Gident. Possibilita a geração de várias barras com mesmas características em um único comando.

- Grest: Refere-se às restrições nos apoios. Define o número do nó e os deslocamentos a serem restringidos. O número 1 indica restrição e 0 para liberar.

Gcar: Gerador de cargas. Iniciando com a letra "n", que significa nó, o número do nó e as cargas são digitadas em seguida. Caso contrário, o primeiro número indica a barra, digitando-se em seguida o carregamento. No final da linha deve-se indicar se a carga é no plano XY ou no plano YZ.

Em cada seção, o editor percebe a palavra chave digitada e de imediato mostra na tela, à direita, a 
sequiência a ser obedecida para se introduzir os dados. A FIGURA 3.25 mostra a ajuda durante a seção Gnos.

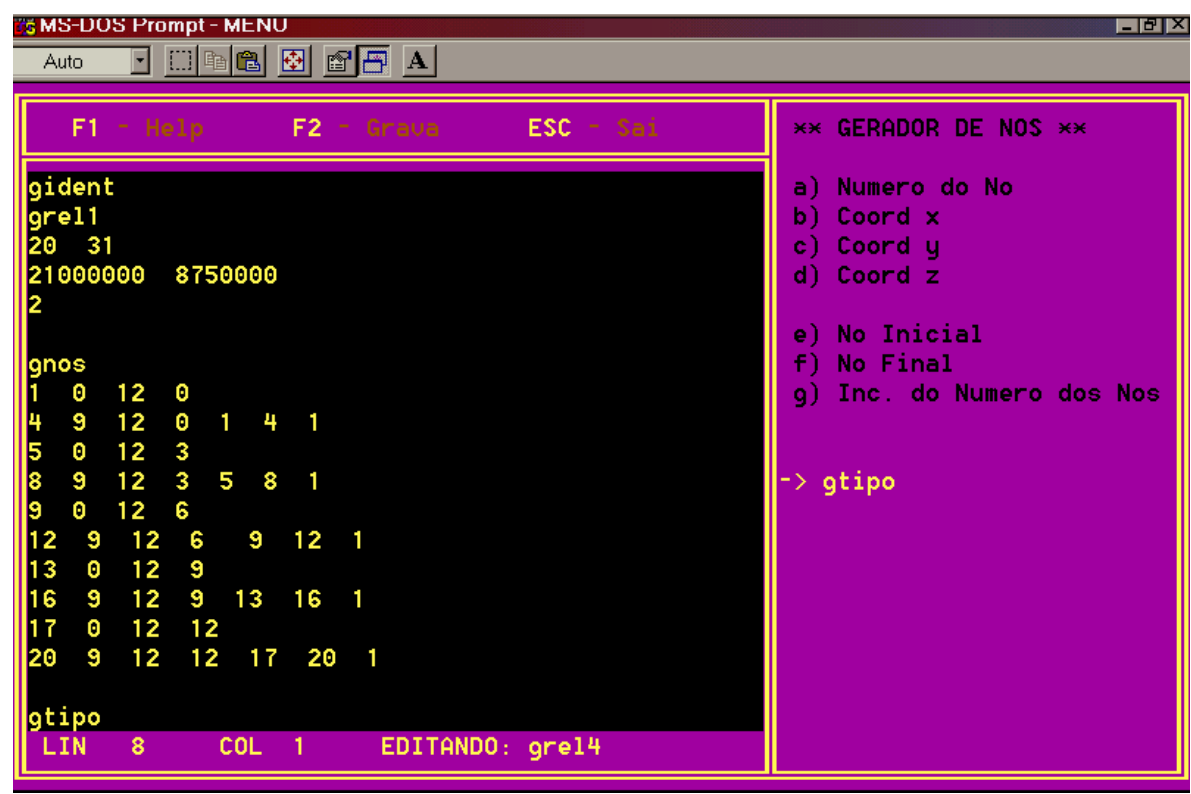

FIGURA 3.25 - Editor de texto e Ajuda durante a edição em Gnos

Cada subestrutura será digitada neste editor $e$, posteriormente, todos os dados introduzidos serão adequadamente gravados em arquivos, para que o programa de análise estrutural possa interpretá-los e processalos. 


\section{CAPÍTULO 4 - EXEMPLOS}

O software desenvolvido tem como objetivo principal a utilização da Técnica das subestruturas em análise linear.

Este capítulo visa demonstrar, através de exemplos, a versatilidade desta técnica, no sentido de permitir uma melhor organização na idealização estrutural, como também no dimensionamento da memória utilizada pelo computador, conforme objetivos propostos para este trabalho.

Em cada exemplo será apresentada a discretização individual de cada subestrutura, como também a estrutura principal que elas formam. Os exemplos estarão acompanhados com detalhes do editor gráfico.

o exemplo-1 simplesmente confere a união de 2 tipos estruturais diferentes. No exemplo-2 predomina o quesito de menor dimensão na memória utilizada pelo computador, como também a facilidade de geração gráfica para estes tipos estruturais.

O exemplo-3 mostra uma união mais complexa de diferentes tipos estruturais. 


\section{1 - Exemplo-1}

Consiste em uma estrutura plana, formada por duas treliças e três pilares (elementos de pórtico).

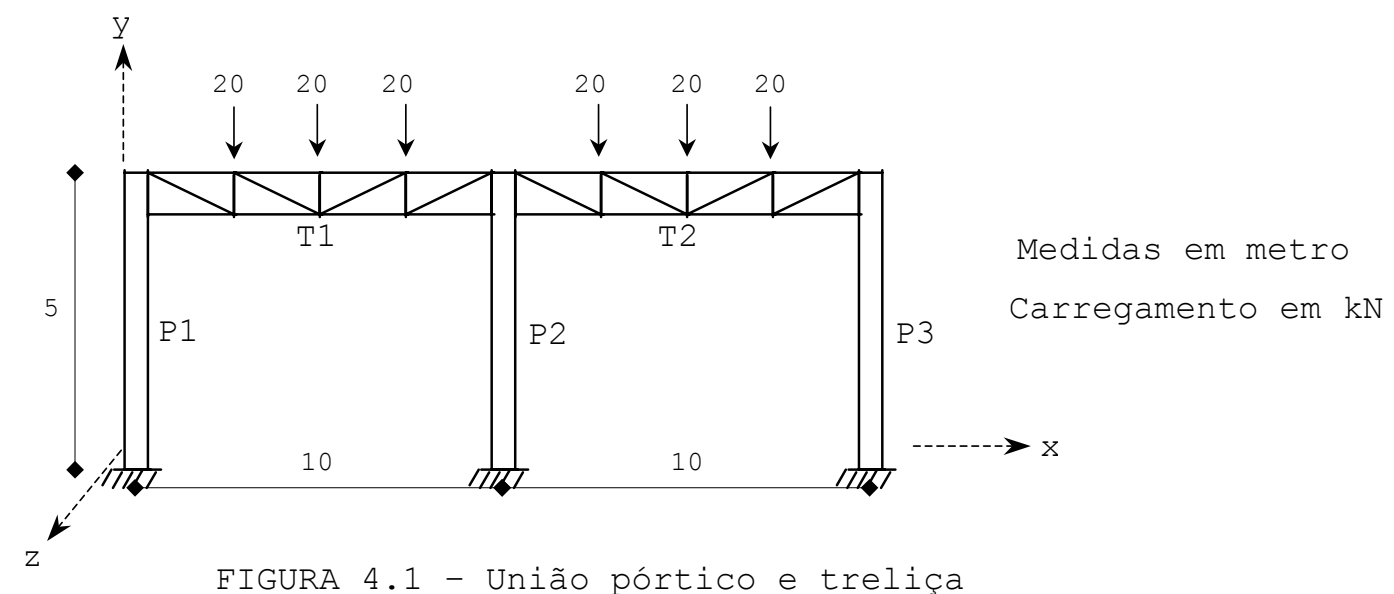

Subestruturas 1, 2 e 3, são respectivamente os pilares P1, P2 e P3:

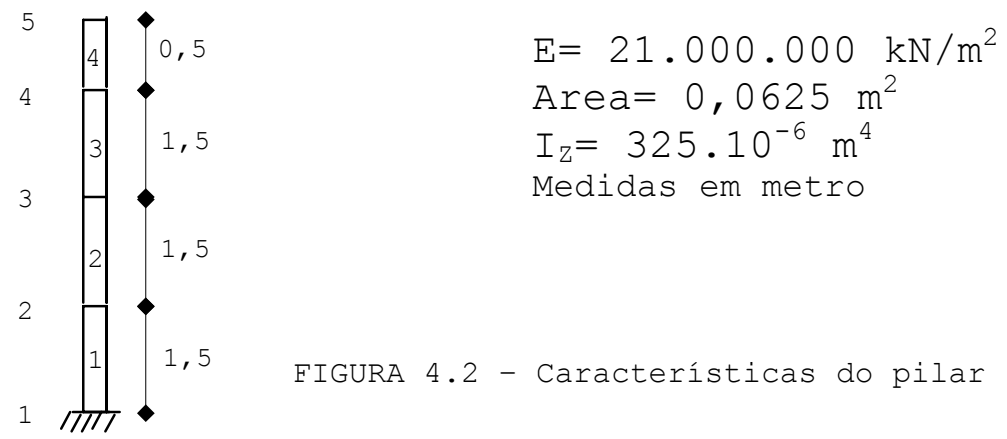

Subestruturas 4 e 5 são, respectivamente, as treliças T1 e T2:

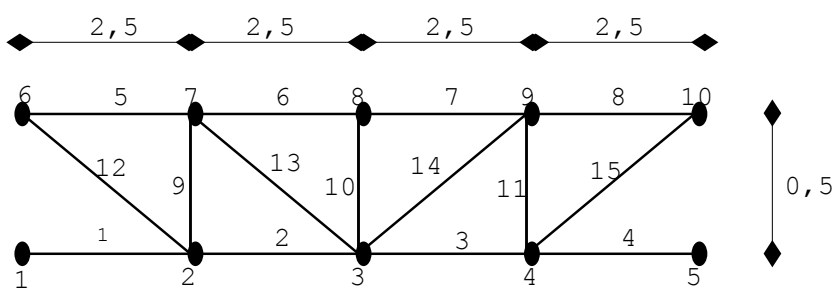

$\mathrm{E}=210.000 .000 \mathrm{kN} / \mathrm{m}^{2}$

$\mathrm{A}_{\text {barras }}=1$

Medidas em metro

FIGURA 4.3 - Características da treliça 
As subestruturas básicas que compõe a estrutura principal são desenhadas inicialmente, conforme FIGURA 4.4 .

Para a definição da treliça (em azul), o comando "dsub", do programa AutoLisp, irá solicitar que suas barras sejam selecionadas. Em seguida irá acrescentá-las a uma lista (vetor) correspondente à esta subestrutura. O mesmo procedimento é adotado para a definição do pilar (em vermelho) .

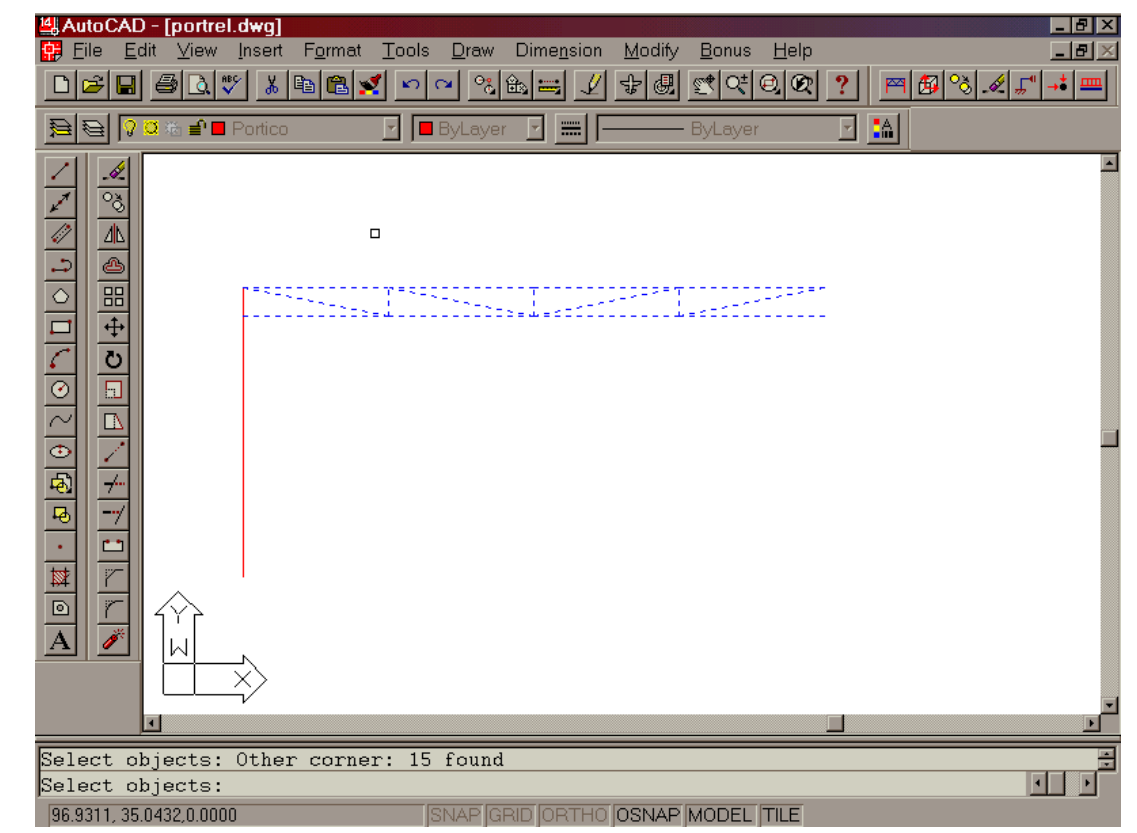

$\begin{aligned} \text { FIGURA 4.4- } & \text { Barras selecionadas para definição da subestrutura } \\ & \text { treliça }\end{aligned}$

Em seguida, o comando "dgeo" irá solicitar que barras sejam selecionadas para acrescentar a elas suas características (Área, $I_{x}, I_{y}, I_{z}, \alpha$ ).

Em todas as funções desenvolvidas, os dados solicitados e as informações emitidas pelo programa são visualizados na barra inferior do AutoCad.

Os carregamentos, nos nós da treliça, são introduzidos através do comando "cargno", que irá 
solicitar a seleção dos nós a receberem os carregamentos, conforme FIGURA 4.5.

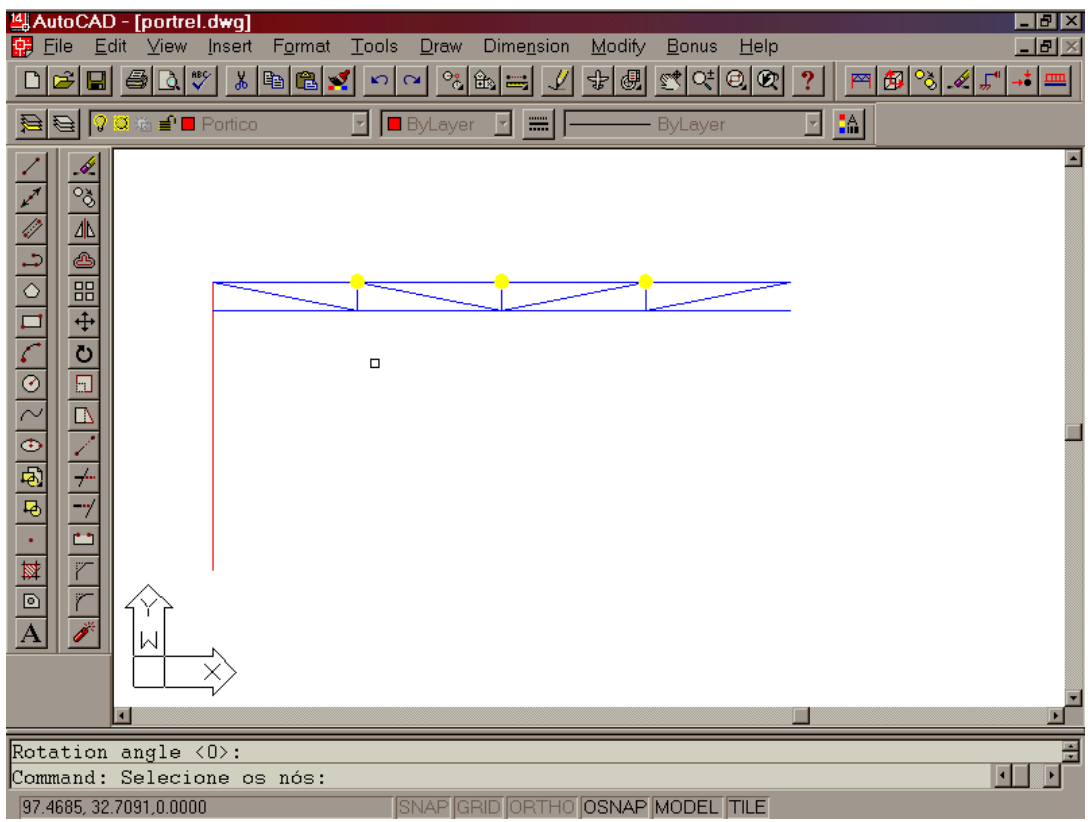

FIGURA 4.5 - Nós selecionados para receberem carregamentos.

Para compor a estrutura completa, através destas subestruturas já definidas, utiliza-se o comando "scopy". Ele irá copiar uma subestrutura já definida para outra posição, acrescentando-a à lista que contém subestruturas definidas. Irá copiar também as características das barras e os carregamentos já implantados.

Após a cópia das treliças e pilares, a estrutura final é: 


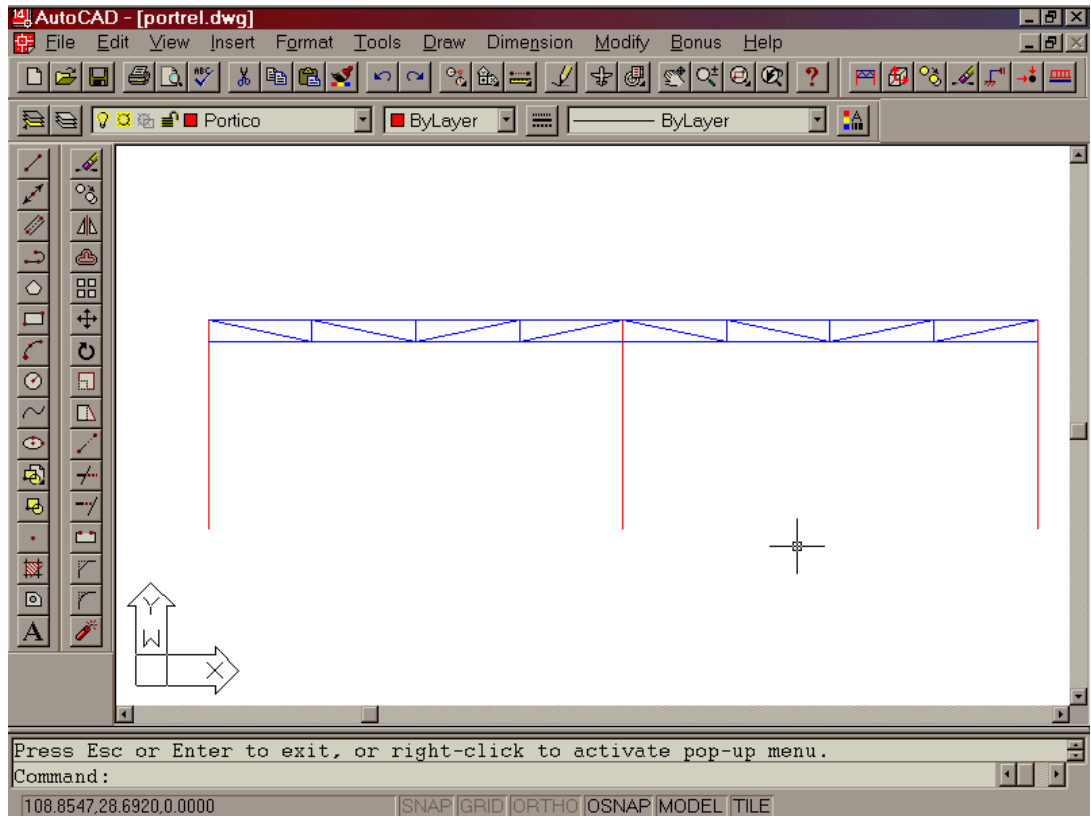

FIGURA 4.6 - Estrutura completa idealizada com a união de subestruturas já discretizadas.

Finalizando, o programa grava todos os dados para posterior durante a análise estrutural pelo programa desenvolvido em linguagem pascal.

Somente os deslocamentos $F_{x}$ e $F_{y}$ dos nós de união serão compatibilizados, apesar do programa tratá-los com estruturas tridimensionais, pois os carregamentos estão contidos no plano da estrutura.

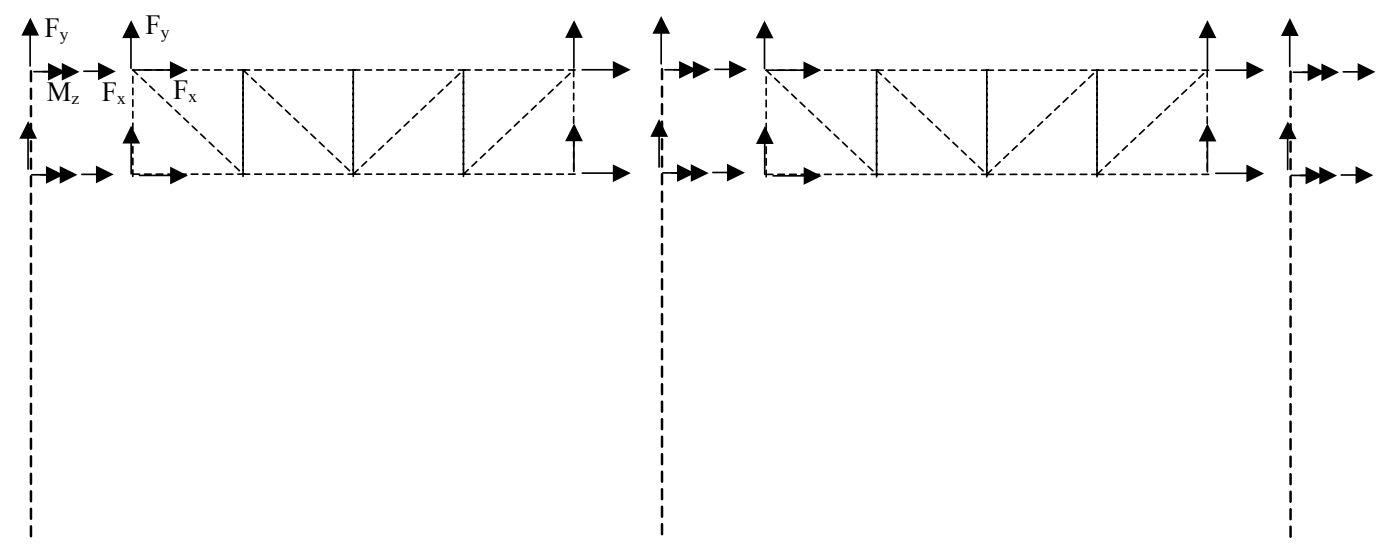

Figura 4.7 - Deslocamentos característicos de cada subestrutura nos nós de união. 
Cada subestrutura será analisada individualmente pelo programa, e as coordenadas comuns entre elas serão detectadas automaticamente.

Esta estrutura possui 27 nós, num total de 162 deslocamentos ( 6 por nó) a serem determinados por um programa de análise tridimensional genérico.

Com a divisão em subestruturas, cada pilar sendo uma subestrutura com 5 nós, irá tratar 30 deslocamentos, 6 deslocamentos por nó ao ser analisado pela subrotina para pórticos. A subrotina para tratamento de treliças irá lidar com 30 deslocamentos (3 por nó) ao analisar cada treliça, com 10 nós cada.

A matriz $\mathrm{S}_{\mathrm{C}}$ irá trabalhar com 36 deslocamentos ( 6 nós de união).

Após a análise da estrutura, temos os resultados para cada subestrutura:

\begin{tabular}{|c|c|c|c|c|c|c|c|}
\hline \multicolumn{2}{|c|}{ Sub.1 } & (P1) & \multicolumn{3}{|c|}{ Solicitações } & \multicolumn{3}{c|}{ Deslocamentos } \\
\hline Bar & Nó & $\mathrm{N}(\mathrm{kN})$ & $\mathrm{Q}(\mathrm{kN})$ & $\mathrm{M}(\mathrm{kN} . \mathrm{m})$ & $\mathrm{D}_{\mathrm{X}}(\mathrm{m})$ & $\mathrm{D}_{\mathrm{Y}}(\mathrm{m})$ & $\mathrm{D}_{\mathrm{Z}}(\mathrm{rad})$ \\
\hline \multirow{2}{*}{1} & 1 & 23,13 & $-0,19$ & $-0,28$ & 0 & 0 & 0 \\
\cline { 2 - 8 } & 2 & $-23,13$ & 0,19 & 0 & $-311 \mathrm{E}-7$ & $-264 \mathrm{E}-7$ & $312 \mathrm{E}-7$ \\
\hline 2 & 2 & 23,13 & $-0,19$ & 0 & $-311 \mathrm{E}-7$ & $-264 \mathrm{E}-7$ & $312 \mathrm{E}-7$ \\
\cline { 2 - 8 } & 3 & $-23,13$ & 0,19 & $-0,28$ & $-627 \mathrm{E}-7$ & $-529 \mathrm{E}-7$ & $7 \mathrm{E}-7$ \\
\hline 3 & 3 & 23,13 & $-0,19$ & 0,28 & $-627 \mathrm{E}-7$ & $-529 \mathrm{E}-7$ & $7 \mathrm{E}-7$ \\
\cline { 2 - 8 } & 4 & $-23,13$ & 0,19 & $-0,56$ & $-24 \mathrm{E}-7$ & $-793 \mathrm{E}-7$ & $-913 \mathrm{E}-7$ \\
\hline 4 & 4 & 23,13 & 1,12 & 0,56 & $-24 \mathrm{E}-7$ & $-793 \mathrm{E}-7$ & $-913 \mathrm{E}-7$ \\
\cline { 2 - 8 } & 5 & $-23,13$ & $-1,12$ & 0 & $501 \mathrm{E}-7$ & $-881 \mathrm{E}-7$ & $-112 \mathrm{E}-6$ \\
\hline
\end{tabular}

TABELA 4.1 - Resultados PILAR1 


\begin{tabular}{|c|c|c|c|c|c|c|c|}
\hline \multicolumn{2}{|c|}{ Sub.2 (P2) } & \multicolumn{3}{|c|}{ Solicitações } & \multicolumn{3}{c|}{ Deslocamentos } \\
\hline Bar & Nó & $\mathrm{N}(\mathrm{kN})$ & $\mathrm{Q}(\mathrm{kN})$ & $\mathrm{M}(\mathrm{kN} \cdot \mathrm{m})$ & $\mathrm{D}_{\mathrm{X}}(\mathrm{m})$ & $\mathrm{D}_{\mathrm{Y}}(\mathrm{m})$ & $\mathrm{D}_{\mathrm{Z}}(\mathrm{rad})$ \\
\hline 1 & 1 & 73,73 & 0 & 0 & 0 & 0 & 0 \\
\cline { 2 - 8 } & 2 & $-73,73$ & 0 & 0 & 0 & $-843 \mathrm{E}-7$ & 0 \\
\hline 2 & 2 & 73,73 & 0 & 0 & 0 & $-843 \mathrm{E}-7$ & 0 \\
\cline { 2 - 9 } & 3 & $-73,73$ & 0 & 0 & 0 & $-168 \mathrm{E}-6$ & 0 \\
\hline 3 & 3 & 73,73 & 0 & 0 & 0 & $-168 \mathrm{E}-6$ & 0 \\
\cline { 2 - 8 } & 4 & $-73,73$ & 0 & 0 & 0 & $-253 \mathrm{E}-6$ & 0 \\
\hline 4 & 4 & 73,73 & 0 & 0 & 0 & $-253 \mathrm{E}-6$ & 0 \\
\cline { 2 - 8 } & 5 & $-73,73$ & 0 & 0 & 0 & $-281 \mathrm{E}-6$ & 0 \\
\hline
\end{tabular}

TABELA 4.2 - Resultados PILAR2

\begin{tabular}{|c|c|c|c|c|c|c|c|}
\hline Sub.3 (P3) & \multicolumn{3}{|c|}{ Solicitações } & \multicolumn{3}{c|}{ Deslocamentos } \\
\hline \multirow{2}{*}{ Bar } & Nó & $\mathrm{N}(\mathrm{kN})$ & $\mathrm{Q}(\mathrm{kN})$ & $\mathrm{M}(\mathrm{kN} \cdot \mathrm{m})$ & $\mathrm{D}_{\mathrm{X}}(\mathrm{m})$ & $\mathrm{D}_{\mathrm{Y}}(\mathrm{m})$ & $\mathrm{M}_{\mathrm{Z}}(\mathrm{rad})$ \\
\hline \multirow{2}{*}{1} & 1 & 23,13 & 0,19 & 0,28 & 0 & 0 & 0 \\
\cline { 2 - 8 } & 2 & $-23,13$ & $-0,19$ & 0 & $311 \mathrm{E}-7$ & $-264 \mathrm{E}-7$ & $-312 \mathrm{E}-7$ \\
\hline \multirow{2}{*}{2} & 2 & 23,13 & 0,19 & 0 & $311 \mathrm{E}-7$ & $-264 \mathrm{E}-7$ & $-312 \mathrm{E}-7$ \\
\cline { 2 - 8 } & 3 & $-23,13$ & $-0,19$ & 0,28 & $627 \mathrm{E}-7$ & $-529 \mathrm{E}-7$ & $-7 \mathrm{E}-7$ \\
\hline \multirow{2}{*}{3} & 3 & 23,13 & 0,19 & $-0,28$ & $627 \mathrm{E}-7$ & $-529 \mathrm{E}-7$ & $-7 \mathrm{E}-7$ \\
\cline { 2 - 8 } & 4 & $-23,13$ & $-0,19$ & 0,56 & $24 \mathrm{E}-7$ & $-793 \mathrm{E}-7$ & $913 \mathrm{E}-7$ \\
\hline \multirow{2}{*}{4} & 4 & 23,13 & $-1,12$ & $-0,56$ & $24 \mathrm{E}-7$ & $-793 \mathrm{E}-7$ & $913 \mathrm{E}-7$ \\
\cline { 2 - 8 } & 5 & $-23,13$ & 1,12 & 0 & $-501 \mathrm{E}-7$ & $-881 \mathrm{E}-7$ & $112 \mathrm{E}-6$ \\
\hline
\end{tabular}

TABELA 4.3 - Resultados PILAR3

As tabelas acima mostram os resultados dos pilares, que o programa emitiu.

As tabelas abaixo mostram os resultados para as treliças. 


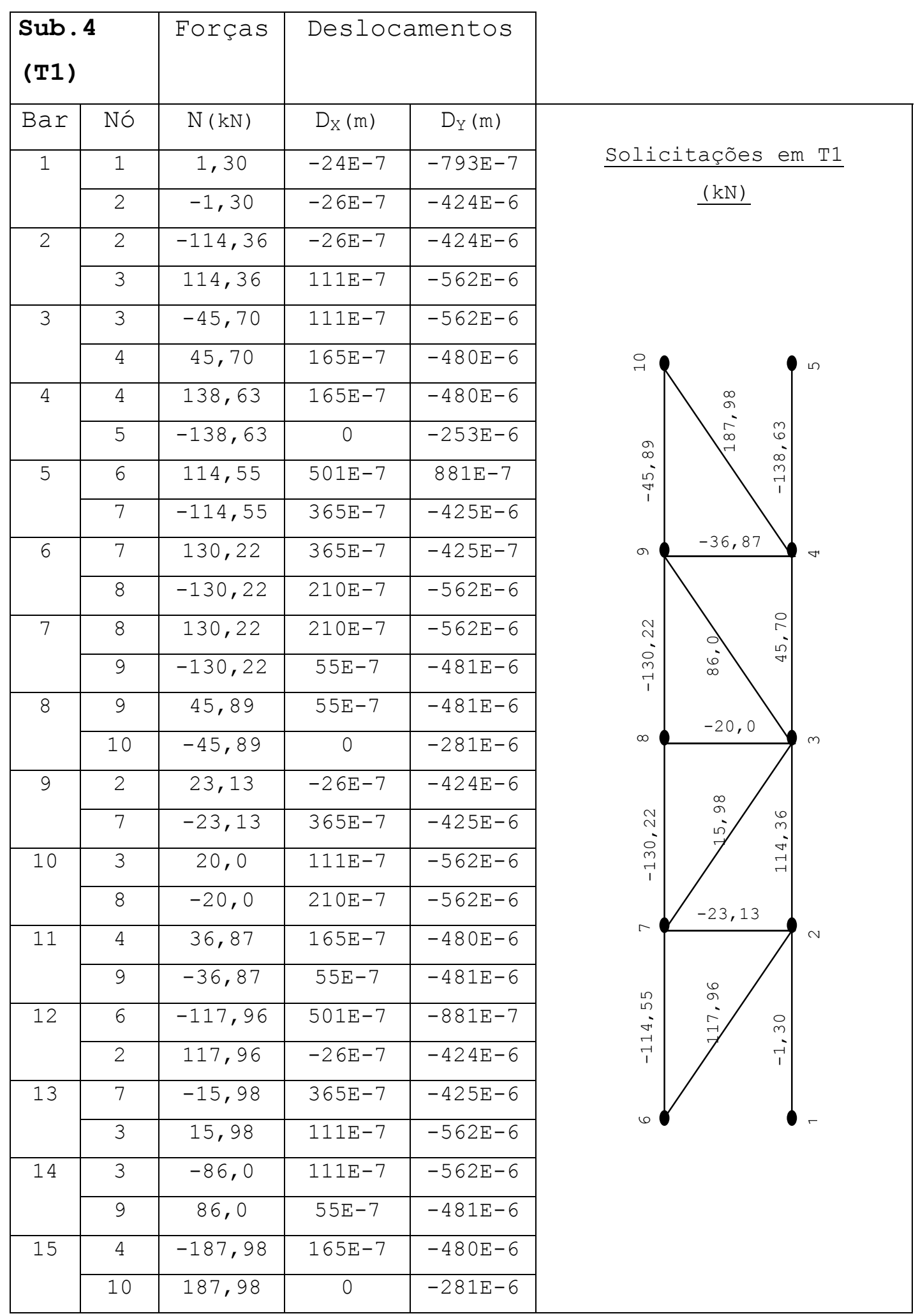

TABELA 4.4 - Resultados TRELIÇA1 


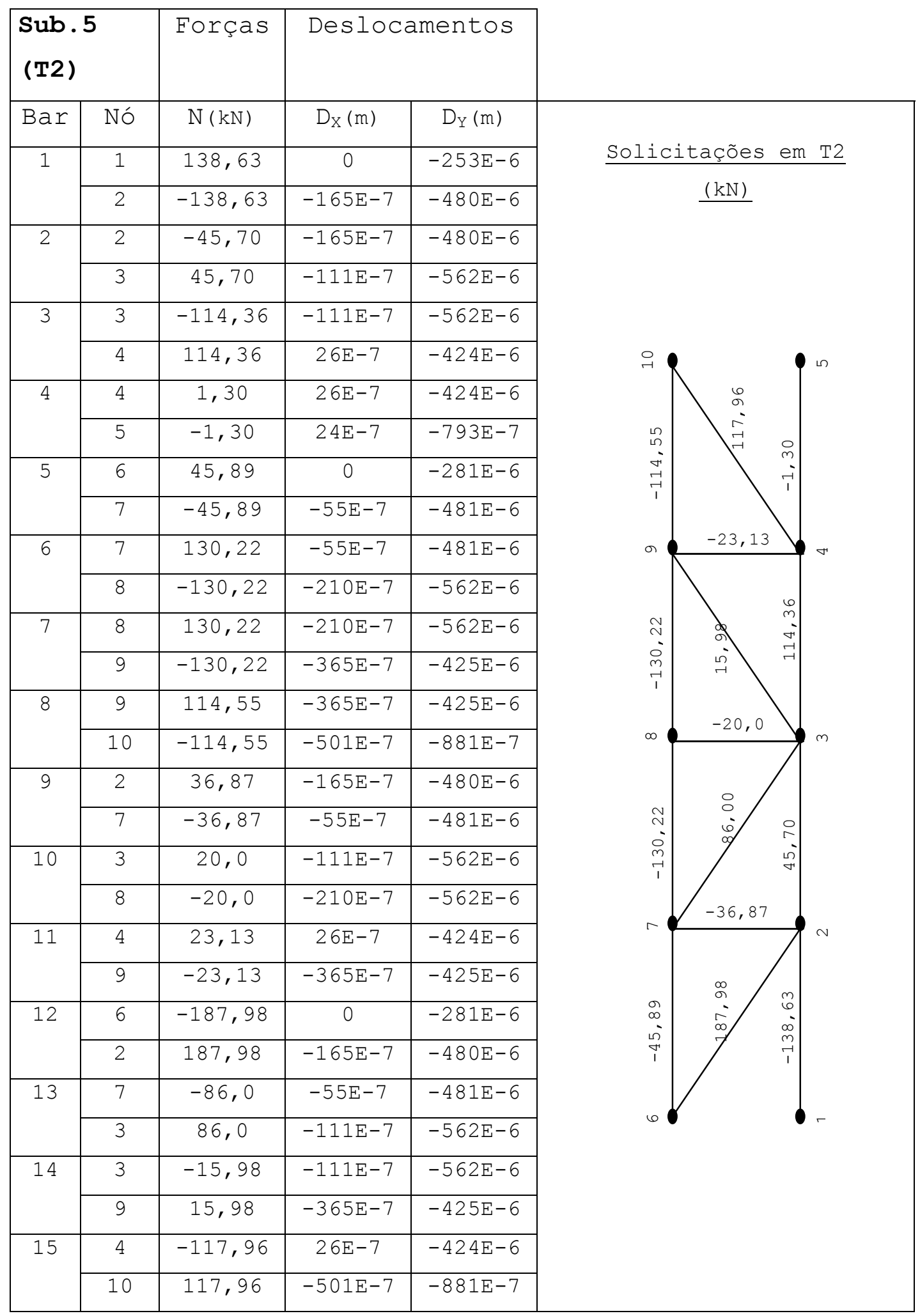

TABELA 4.5 - Resultados TRELIÇA2 
Verificando os resultados das subestruturas, nota-se que as ações de extremidades e os deslocamentos nos nós de união estão compatibilizados. 


\section{2 - Exemplo-2}

Um prédio com 8 pavimentos e 4 pilares, abaixo, será analisado considerando que cada pilar constituirá uma subestrutura, e cada grelha, que forma um pavimento, também será uma subestrutura. Ambas são subestruturas tridimensionais do tipo pórtico.
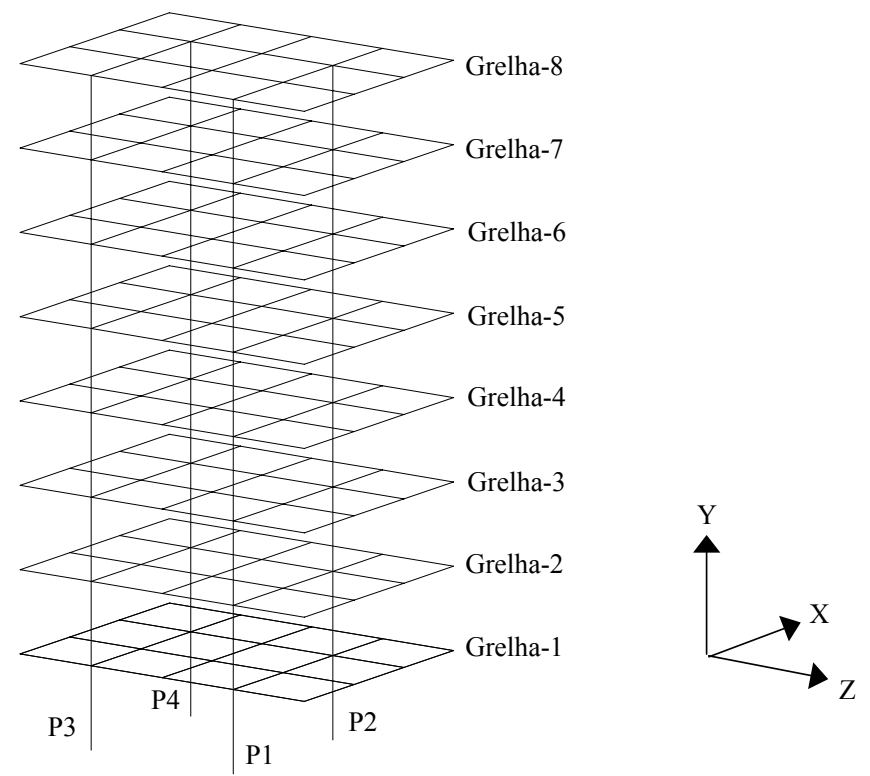

Figura 4.8 - Prédio com 8 pavimentos e sua orientação global.

Por simplificação, todas as vigas da grelha terão a mesma seção transversal e o mesmo carregamento será aplicado a todas elas. Os pilares também terão a mesma seção transversal. A planta de fôrma e as características técnicas das vigas estão na figura abaixo. 


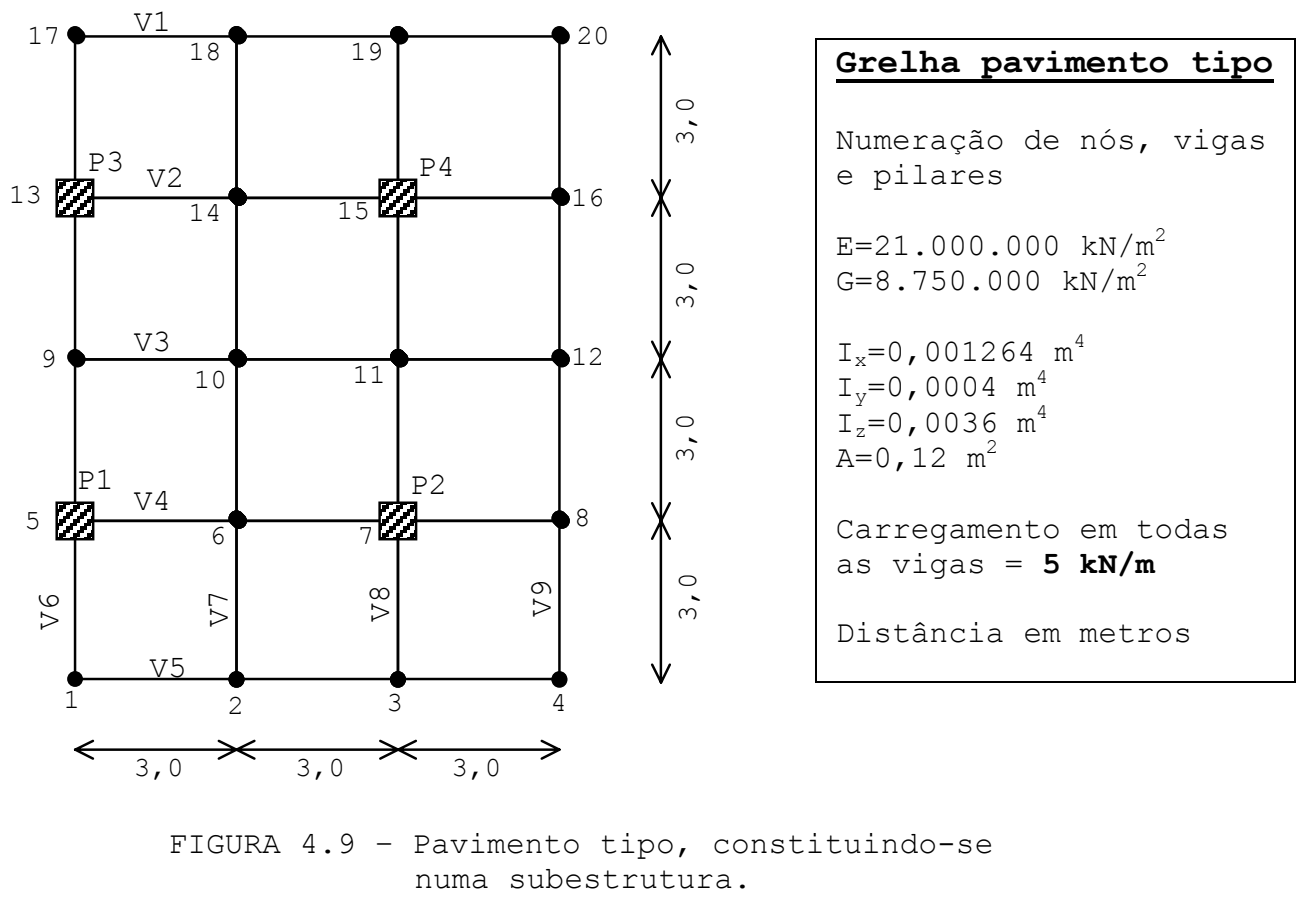

Cada pilar será considerado uma subestrutura. A figura abaixo mostra o pilar padrão e suas características técnicas.

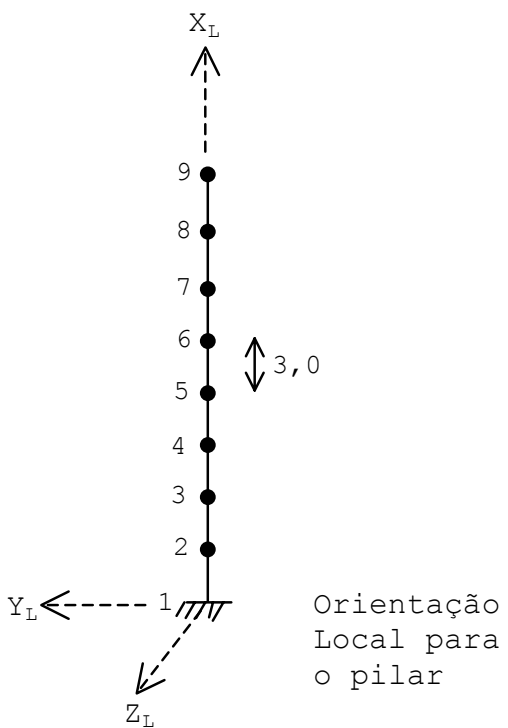

$$
\begin{aligned}
& \text { Pilar Tipo } \\
& \text { Numeração de nós e } \\
& \text { altura de pavimento } \\
& E=21.000 .000 \mathrm{kN} / \mathrm{m}^{2} \\
& G=8.750 .000 \mathrm{kN} / \mathrm{m}^{2} \\
& I_{\mathrm{x}}=0,00233 \mathrm{~m}^{4} \\
& I_{\mathrm{y}}=0,000667 \mathrm{~m}^{4} \\
& I_{\mathrm{z}}=0,01667 \mathrm{~m}^{4} \\
& A=0,20 \mathrm{~m}^{2} \\
& \text { Distancia em metro }
\end{aligned}
$$

FIGURA 4.10 - Características do pilar 
A figura abaixo mostra os dois tipos de subestruturas que ocorrem, já definidas pelo interpretador gráfico com o comando "dsub".

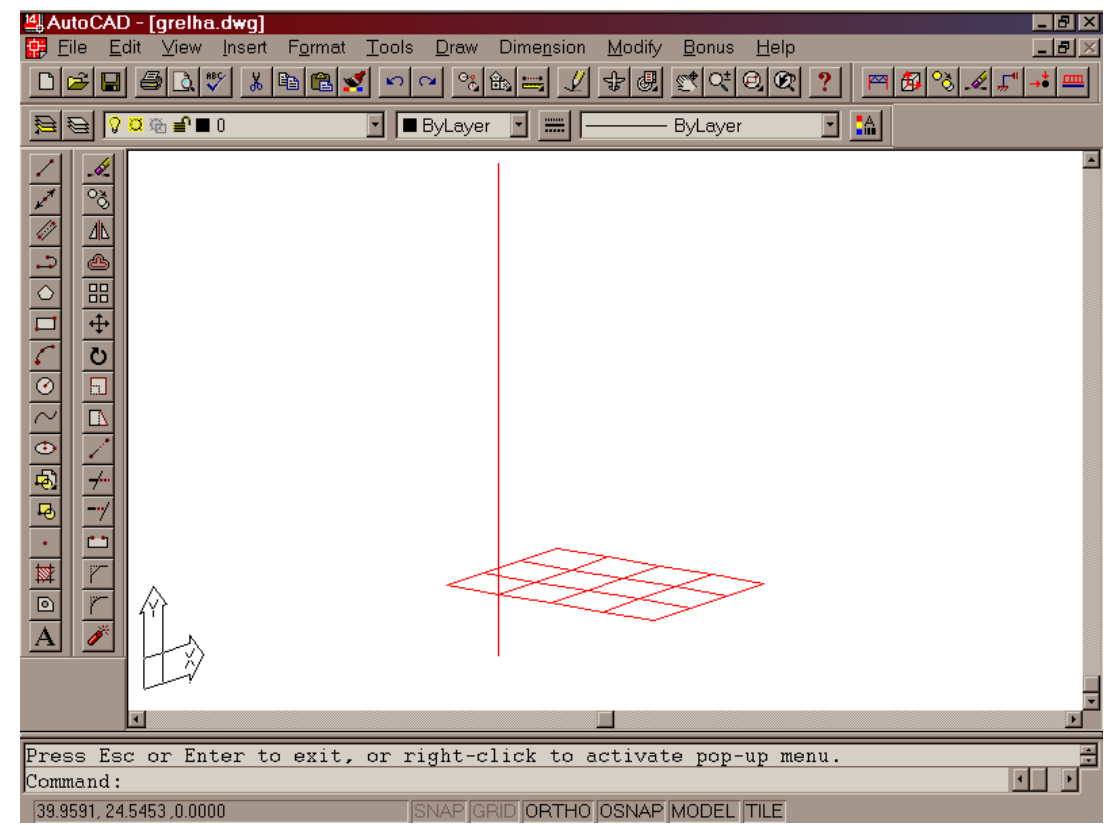

FIGURA 4.11 - Principais subestruturas já definidas

Com os comandos apropriados, são inseridos os dados relativos às seções transversais ("dgeo"), carregamentos nas barras ("carga"), para todas as subestruturas. Desta forma, quando se utilizar o comando "scopy", que copia subestruturas para outra posição, as características das barras e carregamentos também serão copiados.

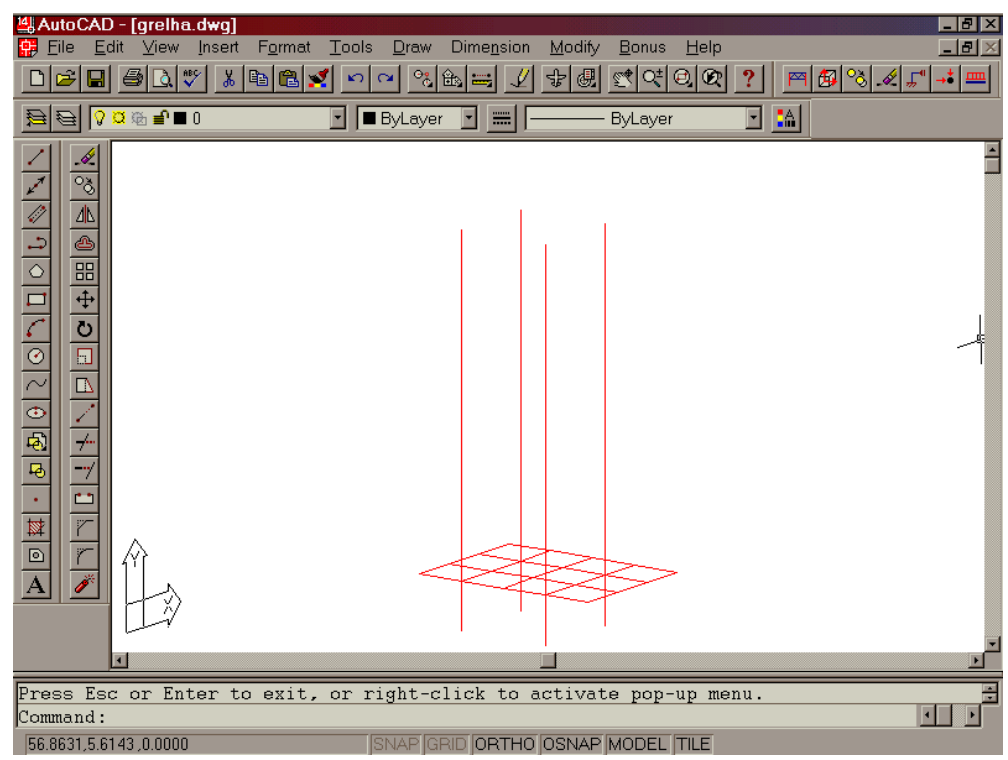

Figura 4.12 - Pilares definidos com "scopy" 
Finalizando a discretização com a cópia das grelhas e definição dos nós de apoio, conforme FIGURA 4.13, o programa irá gravar todos os dados em arquivos para posterior utilização pelo programa de análise. Cada subestrutura terá seu arquivo próprio de dados.

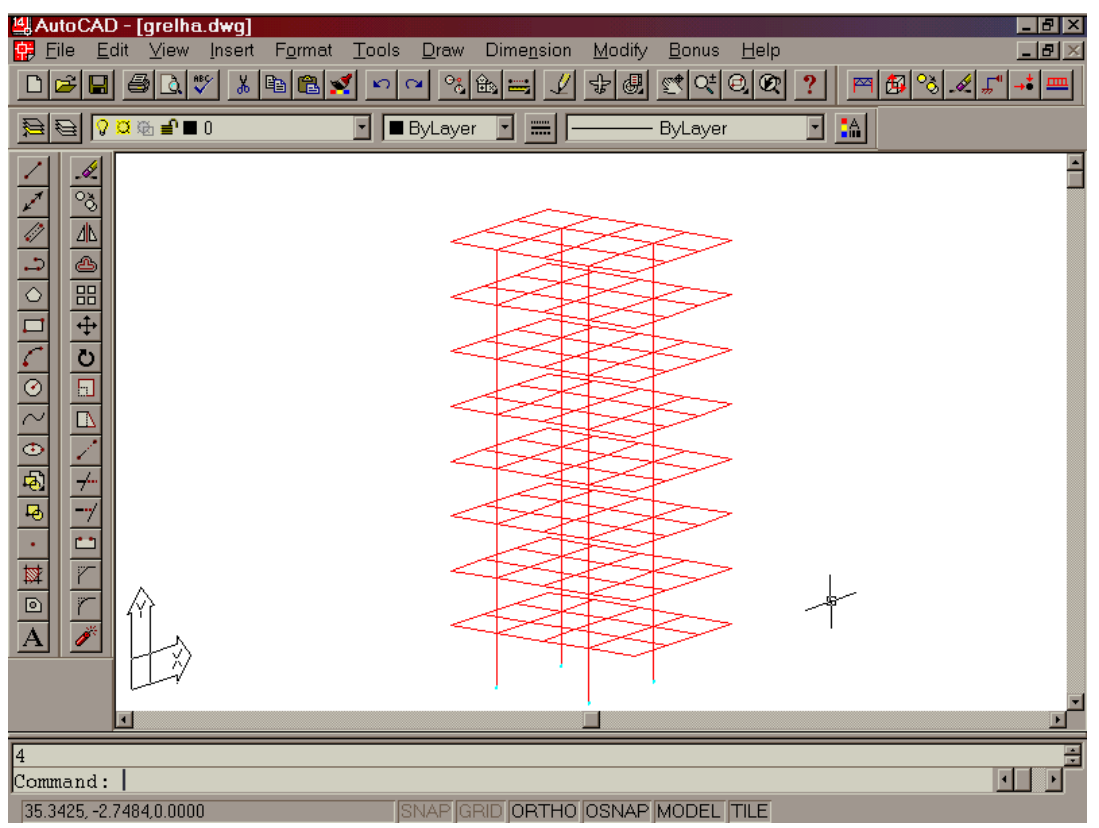

FIGURA 4.13 - Estrutura completa formada pela união de subestruturas previamente definidas.

Durante a análise individual, para cada subestrutura, cada grelha formará 240 deslocamentos a serem determinados ( 6 por nó), enquanto cada pilar formará 54 deslocamentos como incógnitas.

A matriz $\mathrm{S}_{\mathrm{C}}$, conterá os deslocamentos de todos os nós de união entre todas as subestruturas, totalizando 192 deslocamentos para análise. 


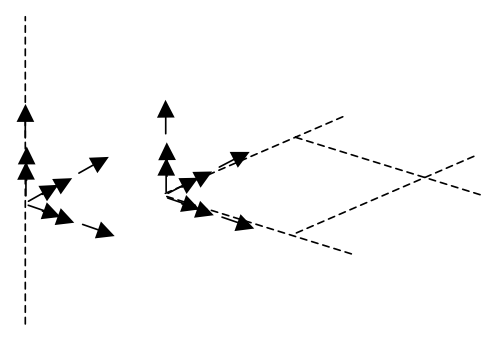

FIGURA 4.14 - Compatibilizar 6 deslocamentos em cada nó de união, entre pilares e vigas.

Este prédio analisado sem partição em subestruturas totalizará 984 deslocamentos a serem determinados. A diminuição no tamanho das equações é considerável em se particionando em subestruturas.

Este tipo de estrutura é bem adequado à utilização do método das subestruturas. A semelhança estrutural entre as grelhas, em todos os sentidos, permite uma maneira rápida e prática de serem lançadas pelo interpretador gráfico.

Após a análise, os resultados para o Pilar P2 são mostrados a seguir: 


\begin{tabular}{|c|c|c|c|c|c|c|c|}
\hline \multicolumn{2}{|c|}{ Pilar (P2) } & \multicolumn{3}{|c|}{ Forças (kN) } & \multicolumn{3}{|c|}{ Momentos (kN.m) } \\
\hline Bar & Nó & $\mathrm{N}_{\mathrm{XL}}$ & $\mathrm{F}_{\mathrm{YL}}$ & $\overline{\mathrm{F}_{\mathrm{ZL}}}$ & $\mathrm{M}_{\mathrm{XL}}$ & $\mathrm{M}_{Y_{\mathrm{L}}}$ & $\mathrm{M}_{\mathrm{ZI}}$ \\
\hline \multirow[t]{2}{*}{1} & 1 & 1377,56 & $-2,36$ & $-7,07$ & 0 & 7,09 & 49,87 \\
\hline & 2 & $-1377,56$ & 2,36 & 7,07 & 0 & 14,11 & $-56,96$ \\
\hline \multirow[t]{2}{*}{2} & 2 & 1211,32 & $-3,40$ & $-13,30$ & 0 & 20,29 & 22,46 \\
\hline & 3 & $-1211,32$ & 3,40 & 13,30 & 0 & 19,60 & $-32,67$ \\
\hline \multirow[t]{2}{*}{3} & 3 & 1040,70 & $-3,03$ & $-12,74$ & 0 & 19,07 & 12,11 \\
\hline & 4 & $-1040,70$ & 3,03 & 12,74 & 0 & 19,16 & $-21,20$ \\
\hline \multirow[t]{2}{*}{4} & 4 & 868,21 & $-2,96$ & $-12,82$ & 0 & 19,23 & 6,54 \\
\hline & 5 & $-868,21$ & 2,96 & 12,82 & 0 & 19,23 & $-15,42$ \\
\hline \multirow[t]{2}{*}{5} & 5 & 694,95 & $-2,94$ & $-12,82$ & 0 & 19,24 & 3,25 \\
\hline & 6 & $-694,95$ & 2,94 & 12,82 & 0 & 19,24 & $-12,07$ \\
\hline \multirow[t]{2}{*}{6} & 6 & 521,35 & $-2,91$ & $-12,85$ & 0 & 19,26 & 0,96 \\
\hline & 7 & $-521,35$ & 2,91 & 12,85 & 0 & 19,29 & $-9,69$ \\
\hline \multirow[t]{2}{*}{7} & 7 & 347,61 & $-2,78$ & $-12,61$ & 0 & 19,06 & $-0,97$ \\
\hline & 8 & $-347,61$ & 2,78 & 12,61 & 0 & 18,77 & $-7,38$ \\
\hline \multirow[t]{2}{*}{8} & 8 & 173,81 & $-4,42$ & $-15,07$ & 0 & 21,15 & $-3,16$ \\
\hline & 9 & $-173,81$ & 4,42 & 15,07 & 0 & 24,06 & $-10,11$ \\
\hline
\end{tabular}

TABELA 4.6 - Ações no pilar P2

\begin{tabular}{|c|c|c|c|c|c|c|}
\hline (P2) & \multicolumn{2}{|c|}{ Deslocamentos $(\mathrm{m}) \cdot 10^{-7}$} & \multicolumn{3}{|c|}{ Rotações (rad) $\cdot 10^{-7}$} \\
\hline Nó & $\mathrm{D}_{\mathrm{X}}$ & $\mathrm{D}_{\mathrm{Y}}$ & $\mathrm{D}_{\mathrm{Z}}$ & $\mathrm{R}_{\mathrm{X}}$ & $\mathrm{R}_{\mathrm{Y}}$ & $\mathrm{R}_{\mathrm{Z}}$ \\
\hline 1 & 0 & 0 & 0 & 0 & 0 & 0 \\
\hline 2 & 6715 & -9840 & 73 & -7520 & 0 & -4578 \\
\hline 3 & 23771 & -18492 & 1 & -6774 & 0 & -6940 \\
\hline 4 & 46536 & -25926 & 1 & -6866 & 0 & -8367 \\
\hline 5 & 72858 & -32127 & 1 & -6866 & 0 & -9308 \\
\hline 6 & 101578 & -37091 & 1 & -6866 & 0 & -9965 \\
\hline 7 & 131970 & -40815 & 2 & -6869 & 0 & -10421 \\
\hline 8 & 163467 & -43298 & 29 & -6583 & 0 & -10696 \\
\hline 9 & 195718 & -44539 & 177 & -9693 & -5 & -10994 \\
\hline
\end{tabular}

TABELA 4.7 - Deslocamentos no pilar P2 
$\underline{\text { Pilar P2 }}$

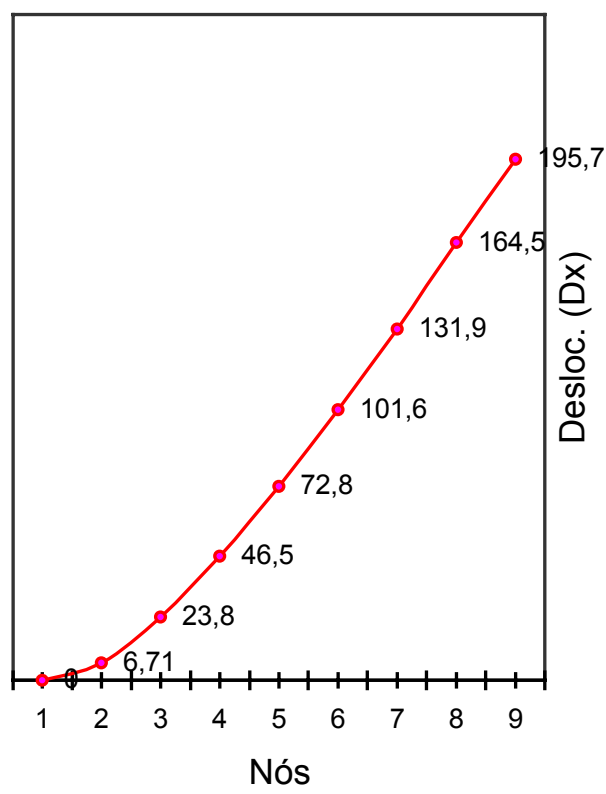

(Gráfico sem escala) $\underline{\text { Pilar P2 }}$

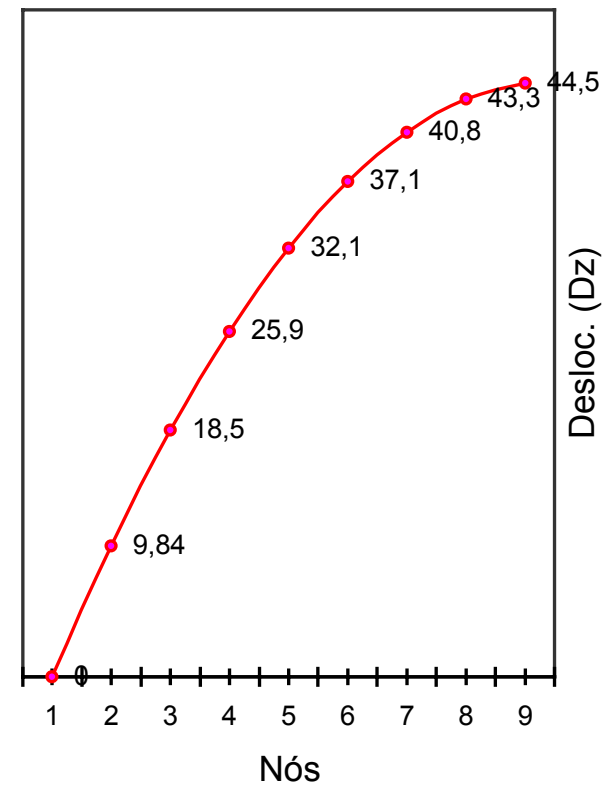

(Gráfico sem escala)

FIGURA 4.15 - Deslocamentos horizontais

A seguir, os resultados da análise somente da viga V4, da grelha 4, que passa por 2 pilares, inclusive P2, para se observar a compatibilização dos deslocamentos nos nós de união.

\begin{tabular}{|c|c|c|c|c|c|c|c|}
\hline \multicolumn{2}{|l|}{ Viga V4 } & \multicolumn{3}{|c|}{ Forças (kN) } & \multicolumn{3}{c|}{ Momentos (kN.m) } \\
\hline Bar & Nó & $N_{\mathrm{XI}_{\mathrm{L}}}$ & $\mathrm{F}_{\mathrm{YL}}$ & $\mathrm{F}_{\mathrm{ZL}}$ & $\mathrm{M}_{\mathrm{XI}}$ & $\mathrm{M}_{\mathrm{YL}}$ & $\mathrm{M}_{\mathrm{ZL}}$ \\
\hline \multirow{2}{*}{1} & 5 & 0 & 16,42 & 0 & 1,69 & 0 & $-4,02$ \\
\cline { 2 - 8 } & 6 & 0 & $-1,42$ & 0 & $-1,69$ & 0 & 30,79 \\
\hline \multirow{2}{*}{2} & 6 & 0 & $-31,74$ & 0 & $-0,66$ & 0 & $-33,63$ \\
\cline { 2 - 8 } & 7 & 0 & 46,74 & 0 & 0,66 & 0 & $-84,08$ \\
\hline \multirow{2}{*}{3} & 7 & 0 & 41,35 & 0 & 0,48 & 0 & 95,48 \\
\cline { 2 - 8 } & 8 & 0 & $-26,35$ & 0 & $-0,48$ & 0 & 6,08 \\
\hline
\end{tabular}




\begin{tabular}{|c|c|c|c|c|c|c|}
\hline V4 & \multicolumn{2}{|c|}{ Deslocamentos $(\mathrm{m}) \cdot 10^{-7}$} & \multicolumn{2}{c|}{ Rotações (rad) $.10^{-7}$} \\
\hline Nó & $\mathrm{D}_{\mathrm{X}}$ & $\mathrm{D}_{\mathrm{Y}}$ & $\mathrm{D}_{\mathrm{Z}}$ & $\mathrm{R}_{\mathrm{X}}$ & $\mathrm{R}_{\mathrm{Y}}$ & $\mathrm{R}_{\mathrm{Z}}$ \\
\hline 5 & 72858 & -11051 & 0 & -4067 & 0 & -9182 \\
\hline 6 & 72858 & -28662 & 0 & -8658 & 0 & -787 \\
\hline 7 & 72858 & -32127 & 0 & -6866 & 0 & -9308 \\
\hline 8 & 72858 & -94502 & 0 & -8180 & 0 & -25559 \\
\hline
\end{tabular}

TABELA 4.9 - Deslocamentos na viga V4 pavimento 4 


\section{3 - Exemplo-3}

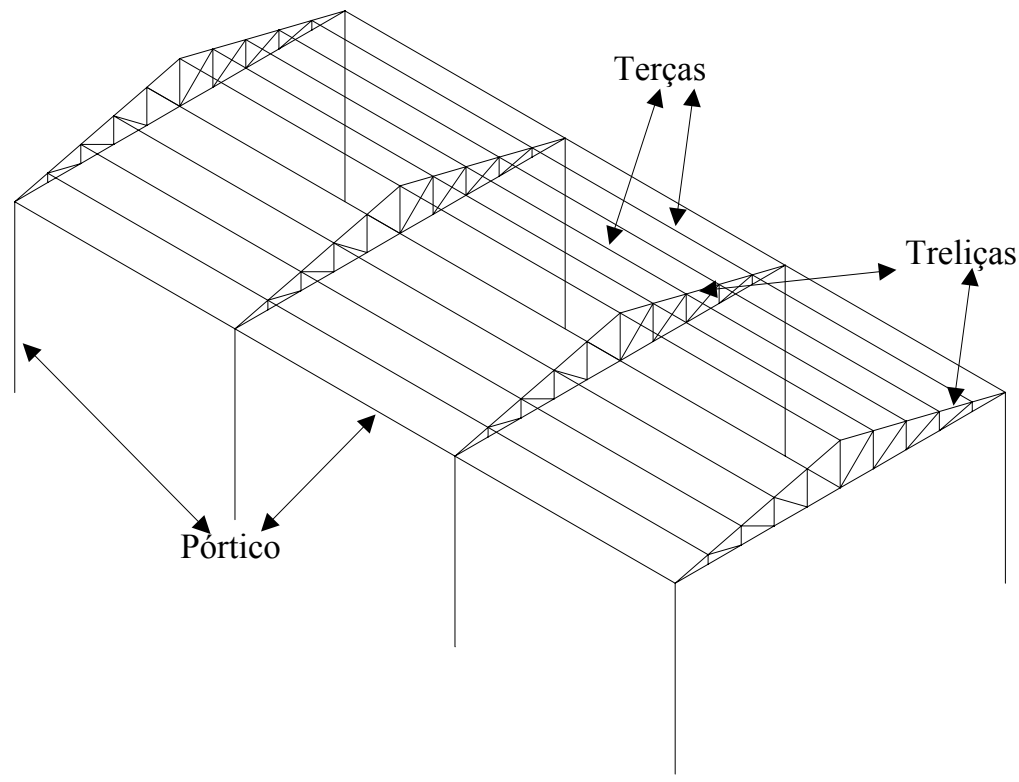

FIGURA 4.16 - Estrutura formada por pórticos, treliças e terças.

Esta estrutura é constituída por:

- Conjunto de 4 treliças iguais, sendo:

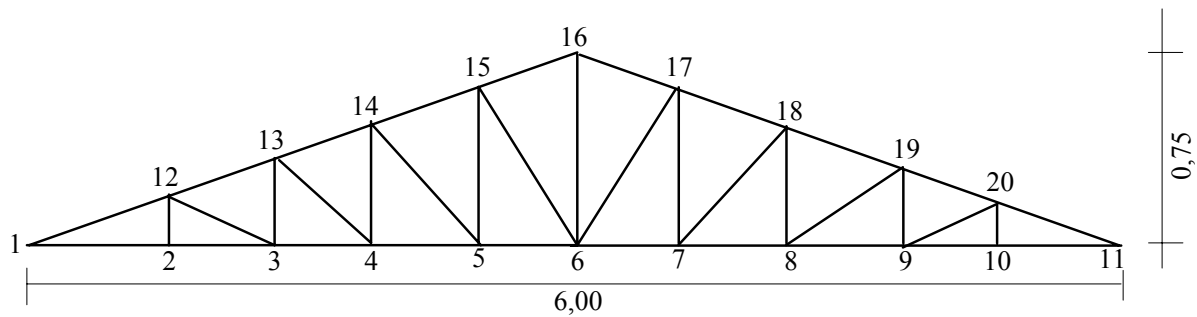

Figura 4.17 - Numeração dos nós da treliça

- Conjunto de 2 pórticos laterais iguais:

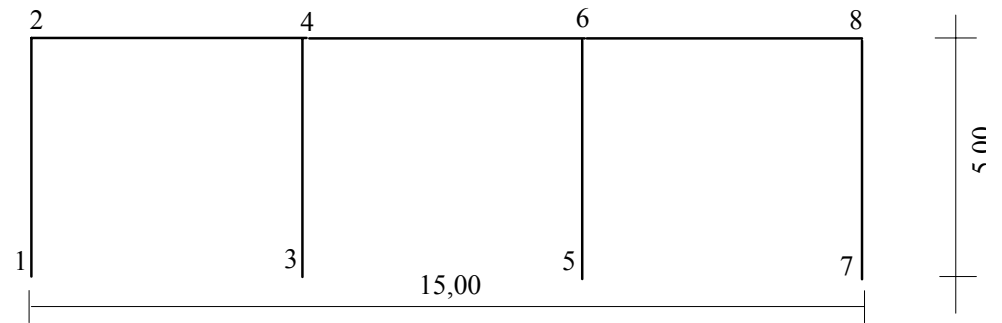

FIGURA 4.18 - Numeração dos nós do pórtico lateral 
- Conjunto com 9 terças iguais, apoiadas sobre as treliças. Serão consideradas peças de pórtico e sendo, neste exemplo, as únicas peças com carregamento. Receberão a carga vertical de $1 \mathrm{kN} / \mathrm{m}$ ao longo de toda a sua extensão.

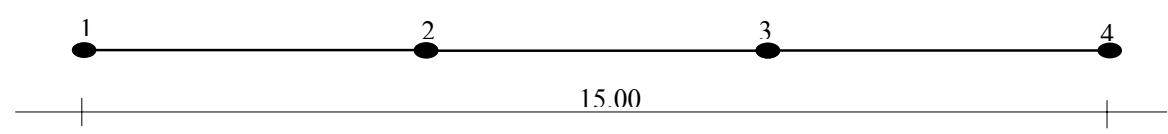

FIGURA 4.19 - Numeração dos nós da Terça.

Iniciando a discretização pela treliça, com a introdução completa de seus dados, vem:

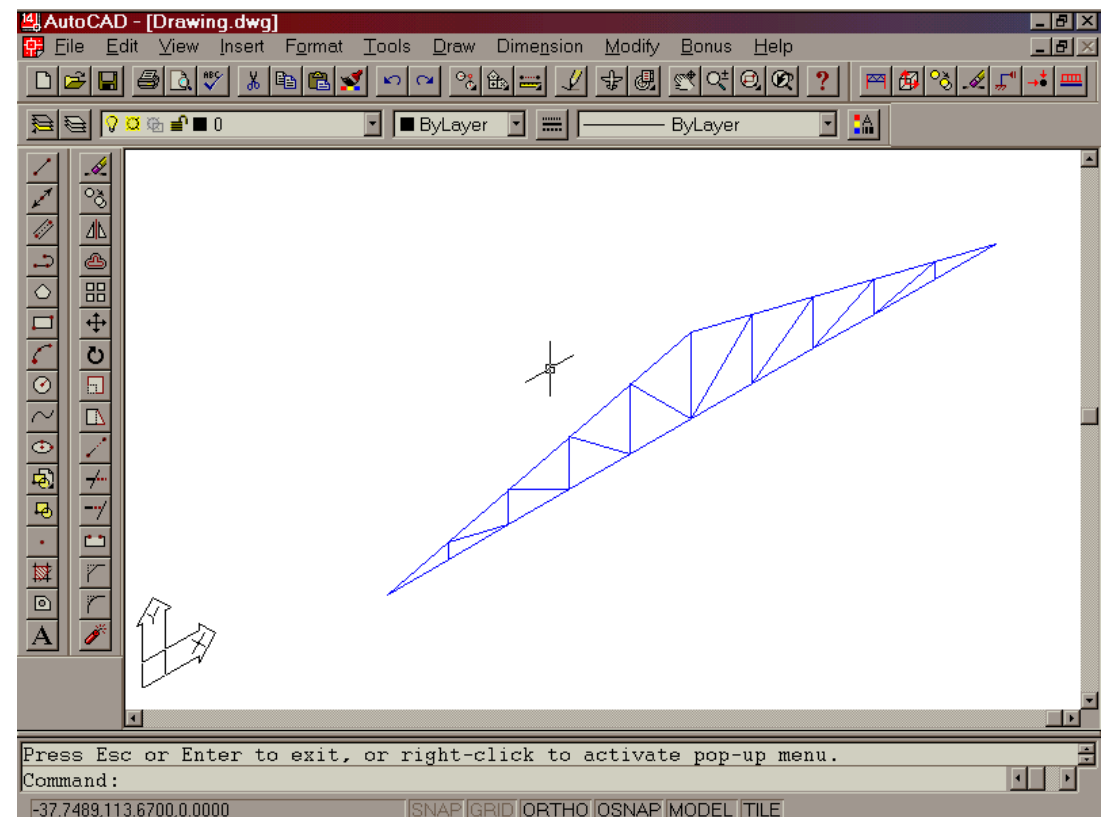

Figura 4.20 - Discretização da treliça.

O comando "scopy" irá copiar as restantes das treliças, juntamente com as características das barras. Logo em seguida o primeiro pórtico é definido e também 
copiado para formar o segundo pórtico, conforme figura abaixo.

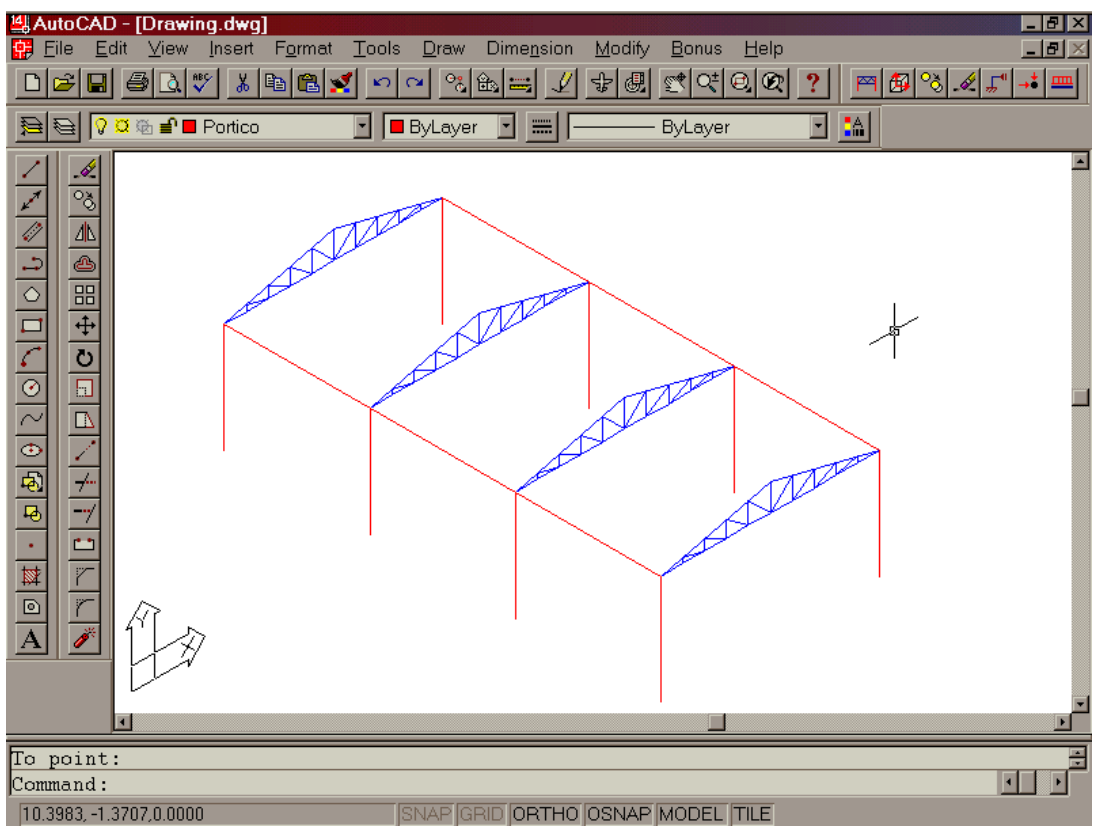

FIGURA 4.21 - Treliças em cor azul e pórticos em vermelho

Finalizando, uma terça é desenhada, definida como subestrutura ("dsub"), suas barras são caracterizadas e os carregamentos aplicados. Em seguida é copiada para todas as outras posições, juntamente com os carregamentos, conforme figura abaixo. 


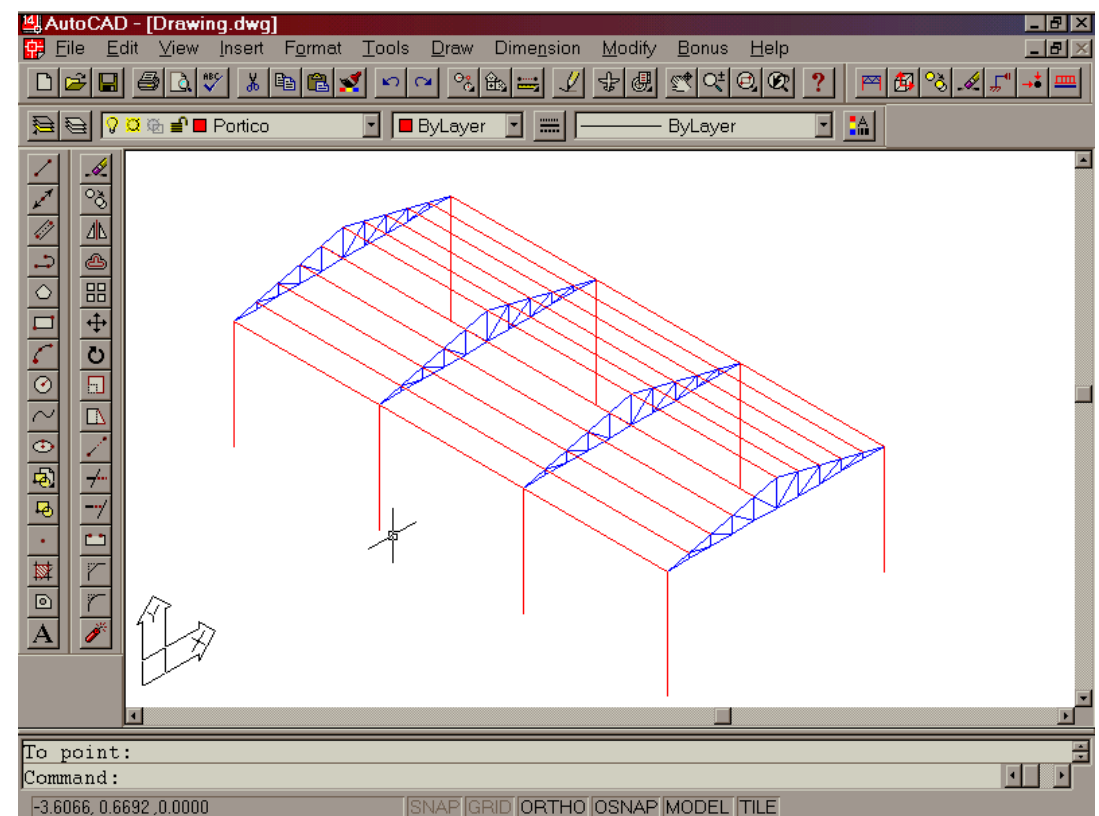

FIGURA 4.22 - Estrutura completa, formada pela união das subestruturas definidas anteriormente.

Cada pórtico foi considerado uma subestrutura, como também cada treliça e cada uma das terças.

A seguir são gravados todos os arquivos necessários ao programa de análise estrutural.

Esta estrutura espacial completa é composta por 88 nós, gerando 528 deslocamentos a serem determinados, por um programa convencional.

Com a divisão em subestruturas, a matriz $\mathbf{s}_{\mathrm{C}}$ foi formada com 264 deslocamentos a serem determinados. Índice maior que os exemplos anteriores devido à quantidade maior de nós em comuns, originados pelas terças.

- programa de análise estrutural gera o seguinte relatório após os calculos, para uma das treliças centrais: 


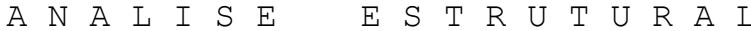

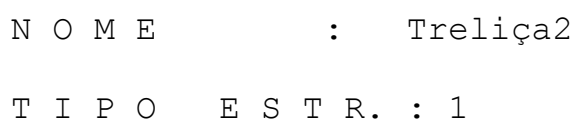

\begin{tabular}{|c|c|c|c|}
\hline & \multicolumn{3}{|c|}{ COORDENADAS DOS NOS } \\
\hline NO & COORD-X & COORD-Y & COORD-Z \\
\hline 1 & 0.00 & 5.00 & 5.00 \\
\hline 2 & 0.60 & 5.00 & 5.00 \\
\hline 3 & 1.20 & 5.00 & 5.00 \\
\hline 4 & 1.80 & 5.00 & 5.00 \\
\hline 5 & 2.40 & 5.00 & 5.00 \\
\hline 6 & 3.00 & 5.00 & 5.00 \\
\hline 7 & 3.60 & 5.00 & 5.00 \\
\hline 8 & 4.20 & 5.00 & 5.00 \\
\hline 9 & 4.80 & 5.00 & 5.00 \\
\hline 10 & 5.40 & 5.00 & 5.00 \\
\hline 11 & 6.00 & 5.00 & 5.00 \\
\hline 12 & 0.60 & 5.15 & 5.00 \\
\hline 13 & 1.20 & 5.30 & 5.00 \\
\hline 14 & 1.80 & 5.45 & 5.00 \\
\hline 15 & 2.40 & 5.60 & 5.00 \\
\hline 16 & 3.00 & 5.75 & 5.00 \\
\hline 17 & 3.60 & 5.60 & 5.00 \\
\hline 18 & 4.20 & 5.45 & 5.00 \\
\hline 19 & 4.80 & 5.30 & 5.00 \\
\hline 20 & 5.40 & 5.15 & 5.00 \\
\hline
\end{tabular}

PROPRIEDADES E GEOMETRIA DAS BARRAS

\begin{tabular}{ccccc} 
MODULO DE ELASTICIDADE : & \multicolumn{3}{l}{ 1.0E+0000 } \\
BARRA & NO-INI & NO-FIN & AREA & COMP \\
& & & & \\
1 & 1 & 2 & 1.00000 & 0.600 \\
2 & 2 & 3 & 1.00000 & 0.600 \\
3 & 3 & 4 & 1.00000 & 0.600 \\
4 & 4 & 5 & 1.00000 & 0.600 \\
5 & 5 & 6 & 1.00000 & 0.600 \\
6 & 6 & 7 & 1.00000 & 0.600 \\
7 & 7 & 8 & 1.00000 & 0.600 \\
8 & 8 & 9 & 1.00000 & 0.600 \\
9 & 9 & 10 & 1.00000 & 0.600 \\
10 & 10 & 11 & 1.00000 & 0.600
\end{tabular}




$\begin{array}{rrrrr}11 & 1 & 12 & 1.00000 & 0.618 \\ 12 & 12 & 13 & 1.00000 & 0.618 \\ 13 & 13 & 14 & 1.00000 & 0.618 \\ 14 & 14 & 15 & 1.00000 & 0.618 \\ 15 & 15 & 16 & 1.00000 & 0.618 \\ 16 & 16 & 17 & 1.00000 & 0.618 \\ 17 & 17 & 18 & 1.00000 & 0.618 \\ 18 & 18 & 19 & 1.00000 & 0.618 \\ 19 & 19 & 20 & 1.00000 & 0.618 \\ 20 & 20 & 11 & 1.00000 & 0.618 \\ 21 & 2 & 12 & 1.00000 & 0.150 \\ 22 & 3 & 13 & 1.00000 & 0.300 \\ 23 & 4 & 14 & 1.00000 & 0.450 \\ 24 & 5 & 15 & 1.00000 & 0.600 \\ 25 & 6 & 16 & 1.00000 & 0.750 \\ 26 & 7 & 17 & 1.00000 & 0.600 \\ 27 & 8 & 18 & 1.00000 & 0.450 \\ 28 & 9 & 19 & 1.00000 & 0.300 \\ 29 & 10 & 20 & 1.00000 & 0.150 \\ 30 & 12 & 3 & 1.00000 & 0.618 \\ 31 & 13 & 4 & 1.00000 & 0.671 \\ 32 & 14 & 5 & 1.00000 & 0.750 \\ 33 & 15 & 6 & 1.00000 & 0.849 \\ 34 & 6 & 17 & 1.00000 & 0.849 \\ 35 & 7 & 18 & 1.00000 & 0.750 \\ 36 & 9 & 19 & 1.00000 & 0.671 \\ 37 & 20 & 1.00000 & 0.618\end{array}$

DESLOCAMENTOS NODAIS

TRANSLACOES

NO

$$
\begin{array}{r}
-152.8163910 \\
-118.1633987 \\
-83.5104065 \\
-54.1347733 \\
-29.7912292 \\
-7.9637408 \\
15.2592449 \\
43.3345490 \\
76.4074554 \\
114.7289734 \\
153.0504608 \\
-7.6975050 \\
27.1974487 \\
34.4413338 \\
27.0639343 \\
7.9256787 \\
-18.3544827 \\
-34.1502686 \\
-33.3198967 \\
-0.9307358
\end{array}
$$

$$
D(Y)
$$$$
-75.8928986
$$$$
-819.4190063
$$$$
-1099.5512695
$$$$
-1247.4908447
$$$$
-1324.6951904
$$$$
-1348.2863770
$$$$
-1355.0314941
$$$$
-1303.6623535
$$$$
-1164.9786377
$$$$
-878.6190796
$$$$
-83.5175705
$$$$
-819.4189453
$$$$
-1098.8916016
$$$$
-1245.6038818
$$$$
-1322.8082275
$$$$
-1338.1011963
$$$$
-1351.3922119
$$$$
-1301.7882080
$$

$-1164.3225098$

$-878.6190796$

\section{D ( Z )}

0.8070270

0.0000000

0.0000000

0.0000000

0.0000000

0.0000000

0.0000000

0.0000000

0.0000000

0.0000000

0.8288099

0.0000000

0.0000000

0.0000000

0.0000000

0.0000000

0.0000000

0.0000000

0.0000000

0.0000000 
ACOES DE EXTREMIDADE

FORCAS

\begin{tabular}{|c|c|c|}
\hline BARRA & NO & $F(X)$ \\
\hline 1 & 1 & -57.75 \\
\hline 1 & 2 & 57.75 \\
\hline 2 & 2 & -57.75 \\
\hline 2 & 3 & 57.75 \\
\hline 3 & 3 & -48.96 \\
\hline 3 & 4 & 48.96 \\
\hline 4 & 4 & -40.57 \\
\hline 4 & 5 & 40.57 \\
\hline 5 & 5 & -36.38 \\
\hline 5 & 6 & 36.38 \\
\hline 6 & 6 & -38.70 \\
\hline 6 & 7 & 38.70 \\
\hline 7 & 7 & -46.79 \\
\hline 7 & 8 & 46.79 \\
\hline 8 & 8 & -55.12 \\
\hline 8 & 9 & 55.12 \\
\hline 9 & 9 & -63.87 \\
\hline 9 & 10 & 63.87 \\
\hline 10 & 10 & -63.87 \\
\hline 10 & 11 & 63.87 \\
\hline 11 & 1 & 63.94 \\
\hline 11 & 12 & -63.94 \\
\hline 12 & 12 & 54.86 \\
\hline 12 & 13 & -54.86 \\
\hline 13 & 13 & 46.17 \\
\hline 13 & 14 & -46.17 \\
\hline 14 & 14 & 41.85 \\
\hline 14 & 15 & -41.85 \\
\hline 15 & 15 & 36.02 \\
\hline 15 & 16 & -36.02 \\
\hline 16 & 16 & 36.01 \\
\hline 16 & 17 & -36.01 \\
\hline 17 & 17 & 44.23 \\
\hline 17 & 18 & -44.23 \\
\hline 18 & 18 & 52.61 \\
\hline 18 & 19 & -52.61 \\
\hline 19 & 19 & 61.23 \\
\hline 19 & 20 & -61.23 \\
\hline 20 & 20 & 70.27 \\
\hline 20 & 11 & -70.27 \\
\hline 21 & 2 & -0.00 \\
\hline 21 & 12 & 0.00 \\
\hline 22 & 3 & -2.20 \\
\hline 22 & 13 & 2.20 \\
\hline 23 & 4 & -4.19 \\
\hline 23 & 14 & 4.19 \\
\hline 24 & 5 & -3.14 \\
\hline 24 & 15 & 3.14 \\
\hline 25 & 6 & -13.58 \\
\hline 25 & 16 & 13.58 \\
\hline
\end{tabular}




$\begin{array}{rrr}26 & 7 & -6.07 \\ 26 & 17 & 6.07 \\ 27 & 8 & -4.16 \\ 27 & 18 & 4.16 \\ 28 & 9 & -2.19 \\ 28 & 19 & 2.19 \\ 29 & 10 & 0.00 \\ 29 & 20 & 0.00 \\ 30 & 12 & 9.07 \\ 30 & 3 & -9.07 \\ 31 & 13 & 9.38 \\ 31 & 4 & -9.38 \\ 32 & 14 & 5.24 \\ 32 & 5 & -5.24 \\ 33 & 15 & 7.96 \\ 33 & 6 & -7.96 \\ 34 & 6 & 11.25 \\ 34 & 17 & -11.25 \\ 35 & 7 & 10.11 \\ 35 & 18 & -10.11 \\ 36 & 8 & 9.31 \\ 36 & 19 & -9.31 \\ 37 & 9 & 9.02 \\ 37 & 20 & -9.02\end{array}$

O relatório para um dos pórticos será:

A $N$ A L I S E E S T R U T U R A L

$\begin{array}{lll}\mathrm{N} O \mathrm{M} \mathrm{E} & & : \text { Pórtico2 } \\ \mathrm{T} \text { I P O } & \text { E S T R. : } 2\end{array}$

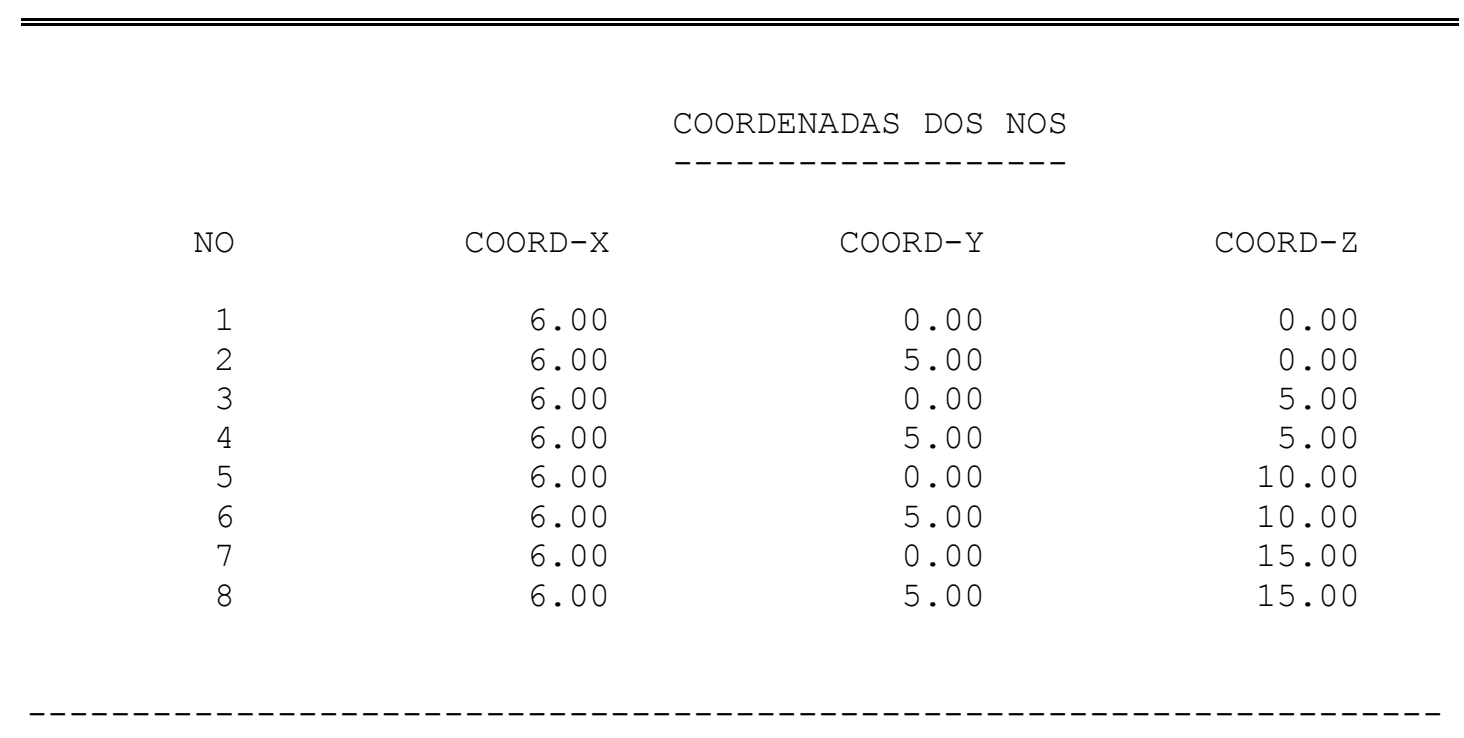


MODULO DE ELASTICIDADE: 1.0E+0000

\begin{tabular}{|c|c|c|c|c|}
\hline BARRA & $\mathrm{NO}-\mathrm{INI}$ & $\mathrm{NO}-\mathrm{FIN}$ & AREA & COMP \\
\hline 1 & 1 & 2 & 1.00000 & 5.000 \\
\hline 2 & 3 & 4 & 1.00000 & 5.000 \\
\hline 3 & 5 & 6 & 1.00000 & 5.000 \\
\hline 4 & 7 & 8 & 1.00000 & 5.000 \\
\hline 5 & 2 & 4 & 1.00000 & 5.000 \\
\hline 6 & 4 & 6 & 1.00000 & 5.000 \\
\hline 7 & 6 & 8 & 1.00000 & 5.000 \\
\hline
\end{tabular}

VINCULACOES

\begin{tabular}{|c|c|c|c|c|c|c|}
\hline NO & REST-X & REST-Y & REST-Z & $\mathrm{MOM}-\mathrm{X}$ & $\mathrm{MOM}-\mathrm{Y}$ & $\mathrm{MOM}-\mathrm{Z}$ \\
\hline 1 & 1 & 1 & 1 & 1 & 1 & 1 \\
\hline 3 & 1 & 1 & 1 & 1 & 1 & 1 \\
\hline 5 & 1 & 1 & 1 & 1 & 1 & 1 \\
\hline 7 & 1 & 1 & 1 & 1 & 1 & 1 \\
\hline
\end{tabular}

DESLOCAMENTOS NODAIS

TRANSLACOES

\begin{tabular}{rr} 
NO & \multicolumn{1}{c}{$D(X)$} \\
1 & 0.0000000 \\
2 & 134.4800873 \\
3 & 0.0000000 \\
4 & 153.0504608 \\
5 & 0.0000000 \\
6 & 153.0528717 \\
7 & 0.0000000 \\
8 & 134.4877319
\end{tabular}

$\mathrm{NO}$

$R(X)$

$$
\begin{array}{r}
0.0000000 \\
1.4542799 \\
0.0000000 \\
0.9541645 \\
0.0000000 \\
-0.9541207 \\
0.0000000
\end{array}
$$

$\mathrm{D}(\mathrm{Y})$

$\mathrm{D}(\mathrm{Z})$

$$
\begin{array}{r}
0.0000000 \\
-73.9712219 \\
0.0000000 \\
-83.5175705 \\
0.0000000 \\
-83.5176620 \\
0.0000000 \\
-73.9715805
\end{array}
$$

0.0000000

1.7391526

0.0000000

0.8288099

0.0000000

$-0.8287013$

0.0000000

$-1.7390445$

\section{ROTACOES}

$$
R(Y)
$$

$R(Z)$

$$
\begin{array}{r}
0.0000000 \\
3.5941844 \\
0.0000000 \\
2.1567209 \\
0.0000000 \\
-2.1557930 \\
0.0000000
\end{array}
$$

0.0000000

$-41.2725716$

0.0000000

$-44.9867668$

0.0000000

$-44.9874573$

0.0000000 


\section{ACOES DE EXTREMIDADE}

FORCAS

\begin{tabular}{ccr} 
BARRA & NO & \multicolumn{1}{c}{$F(X)$} \\
1 & 1 & 14.79 \\
1 & 2 & -14.79 \\
2 & 3 & 16.70 \\
2 & 4 & -16.70 \\
3 & 5 & 16.70 \\
3 & 6 & -16.70 \\
4 & 7 & 14.79 \\
4 & 8 & -14.79 \\
5 & 2 & 0.18 \\
5 & 4 & -0.18 \\
6 & 4 & 0.33 \\
6 & 6 & -0.33 \\
7 & 6 & 0.18 \\
7 & 8 & -0.18
\end{tabular}

$$
\text { F (Y) }
$$$$
\text { F ( Z ) }
$$

$$
\begin{array}{r}
3.00 \\
-3.00
\end{array}
$$$$
3.90
$$$$
-3.90
$$$$
3.90
$$$$
-3.90
$$$$
3.00
$$$$
-3.00
$$$$
0.34
$$$$
-0.34
$$$$
-0.00
$$$$
0.00
$$$$
-0.34
$$$$
0.34
$$

0.18

$$
\begin{array}{r}
-0.18 \\
0.15
\end{array}
$$$$
-0.15
$$$$
-0.15
$$$$
0.15
$$$$
-0.18
$$$$
0.18
$$$$
0.40
$$$$
-0.40
$$$$
0.00
$$$$
-0.00
$$$$
-0.40
$$$$
0.40
$$

MOMENTOS

$\begin{array}{crrrc}\text { BARRA } & \text { NO } & \text { M (X) } & \text { M (Y) } & \text { M (Z) } \\ 1 & 1 & -0.72 & -0.16 & 15.77 \\ 1 & 2 & 0.72 & -0.75 & -0.74 \\ 2 & 3 & -0.43 & -0.18 & 18.74 \\ 2 & 4 & 0.43 & -0.56 & 0.74 \\ 3 & 5 & 0.43 & 0.18 & 18.74 \\ 3 & 6 & -0.43 & 0.56 & 0.74 \\ 4 & 7 & 0.72 & 0.16 & 15.77 \\ 4 & 8 & -0.72 & 0.75 & -0.74 \\ 5 & 2 & 0.74 & -0.72 & 0.75 \\ 5 & 4 & -0.74 & -1.29 & 0.95 \\ 6 & 4 & 0.00 & 0.86 & -0.38 \\ 6 & 6 & -0.00 & -0.86 & 0.38 \\ 7 & 6 & -0.74 & 1.29 & -0.95 \\ 7 & 8 & 0.74 & 0.72 & -0.75\end{array}$

Percebe-se a compatibilização dos deslocamentos entre o nó 11 da treliça e o nó 4 do pórtico:

$\begin{array}{rccc}\text { Nó } & F_{X} & F_{Y} & F_{z} \\ 11 & 153.0504608 & -83.5175705 & 0.8288099 \\ 4 & 153.0504608 & -83.5175705 & 0.8288099\end{array}$




\section{CAPÍTULO 5 - CONCLUSÕES FINAIS}

Este trabalho apresentou a Técnica da Subestruturação seguindo a seqüência :

- Conceito de condensação estática

- Método das Subestruturas (PRZEMIENIECKI, 1968), generalizando a formulação desta técnica para sua aplicação às estruturas.

- Apresentação desta técnica utilizando a Decomposição de Cholesky (RUBINSTEIN,1970), obtendo maior desempenho matemático e computacional.

Os exemplos apresentados confirmam esta técnica como sendo uma ferramenta adequada ao tratamento de estruturas particionadas.

O software, desenvolvido aqui, teve como objetivo principal a implementação desta técnica de forma a propiciar a análise de estruturas constituídas por diferentes tipos de elementos de barra, conforme demonstrado pelos exemplos. Verifica-se também uma melhor organização na discretização da estrutura. 
O exemplo-2 demonstra bem o desempenho desta técnica em diminuir consideravelmente a quantidade de equações a serem resolvidas simultaneamente.

A idealização de interfaces gráficas constitui-se num laborioso e dispendioso trabalho computacional. Atualmente é uma prática constantemente exigida, em acordo com as tendências atuais da informática.

Nesse trabalho, utilizou-se o editor gráfico Autocad, que já possui todas as funções e procedimentos gráficos próprios, eliminando assim árduas etapas em termos de programação.

A interação com seus recursos gráficos tornou-se possível pelo uso de um interpretador de comandos, Autolisp, que permite a criação de variáveis e suas associações com estruturas internas deste editor gráfico.

É uma tendência que vem sendo adotada de maneira significativa na atualidade. A maioria dos editores gráficos desenvolvem interpretadores e compiladores cada vez mais avançados, permitindo que isso se torne uma prática cotidiana.

A maneira como estes editores gráficos organizam suas entidades criadas e os procedimentos seletivos que aplicam em suas operações e funções, propiciou adequadamente a utilização conjunta com o método das subestruturas.

Com isso, um dos objetivos deste trabalho, que era uma melhor organização na discretização estrutural, foi alcançado satisfatoriamente, permitindo vislumbrar este intercâmbio de forma mais completa.

Diante de tais características, criam-se perspectivas em aplicações mais complexas, visando a compatibilização de deslocamentos entre vários outros tipos de elementos estruturais, como elementos finitos, 
por exemplo. Estas estruturas requerem tratamento gráfico mais avançado, que também poderá ser facilitado com a utilização em conjunto de um editor gráfico (Autocad) .

Em termos da análise estrutural, cada tipo estrutural diferente exigirá tratamento por subrotinas adequadas. Com isto, a análise torna-se mais detalhada, como também mais específica aos fenômenos a serem avaliados com a união de diferentes subestruturas.

Da mesma forma, com os dados das subestruturas individualizados, eles podem ser enviados a subrotinas específicas para serem realizados outros tipos de cálculo, como dimensionamentos e detalhamentos de seus elementos.

As perspectivas citadas acima tornam-se subsidios para dar continuidade e aprimoramento ao programa desenvolvido com a utilização desta técnica. 


\section{REFERÊNCIAS BIBLIOGRÁFICAS}

BALCAZAR, E.S.G.(1991). Análise linear de estruturas tridimensionais retangulares pelo método dos elementos finitos utilizando subestruturas. São Carlos. Dissertação (Mestrado)-Escola de Engenharia São Carlos, Universidade de São Paulo.

BEAUFAIT,F. W.; ROWAN JR, W. H.; HOADLEY, P. G.; HACKETT, R. M. (1970).Computer methods of structural analysis. New Jersey, Englewood Cliffs.

BERTOLINO Jr., R.(1986). Técnica de programação e implantação do método dos elementos finitos. Ilha Solteira, UNESP/FEIS. (Acordo SENAI/CESP).

GERE, J.M.; WEAVERJr, W.(1981). Análise de estruturas reticuladas. Rio de Janeiro, Guanabara Dois. 443p.

KRAMER, WILLIAM (1995). Programando em Autolisp. Makron Books, 274p.

LEI, W.; MEEK, J. L. (1993). Multi-level substructuring and its implementation in programming, Advances in Engineering Software, V.16, p.195-202.

MCGUIRE, W.; GALLAGHER, R. H. (1979) . Matrix structural analysis. New York, J. Wiley. 460p.

OMURA, GEORGE. (1992). Dominando o AutoCad Versão 13. Livros Técnicos e Científicos Editora, 963p.

PRZEMIENIECKI, J.S. (1968). Theory of matrix structural analysis. New York, Dover. 468p. 
ROGERS, D. F.; ADAMS, J. A. (1990). Mathematical

elements for computer graphics.2.ed. New York, McGraw Hill. 611p.

ROSEN, R.; RUBINSTEIN, M. F. (1970). Subestructure analysis by matrix decomposition. Journal of Structural Division Proc. ASCE, v.96, n.3 p. 663-70, mar.

RUBINSTEIN, M. F.; ROSEN, R. (1968). Structural analysis by matrix decomposition. Journal of Structural Division. Proc. ASCE, p. 331-43.

SORIANO, H. L. (1981). Sistemas de equações algébricas lineares em problemas estruturais. Lisboa, Laboratório Nacional de Engenharia Civil. (Seminário, 280)

WEAVER JR., W. (1967). Computer programs for structural analysis. Prínceton, D. Van Nostrand. 300p. 